H. Umemura

Nagoya Math. J.

Vol. 144 (1996), 1-58

\title{
GALOIS THEORY OF AIGEBRAIC AND DIFFERENTIAL EQUATIONS
}

\author{
HIROSHI UMEMURA
}

\section{CONTENTS}

$\begin{array}{lr}\text { Introduction } & 1 \\ \S 1 . \text { Preliminaries } & 4 \\ \S 2 . \quad \text { Automorphic extensions } & 18 \\ \S 3 . \quad \text { Relation with the Kolchin theory } & 39 \\ \S 4 . \quad \text { Galois correspondence } & 48 \\ \text { References } & 57\end{array}$

\section{Introduction}

This paper will be the first part of our works on differential Galois theory which we plan to write. Our goal is to establish a Galois Theory of ordinary differential equations. The theory is infinite dimensional by nature and has a long history. The pioneer of this field is S. Lie who tried to apply the idea of Abel and Galois to differential equations. Picard $[\mathrm{P}]$ realized Galois Theory of linear ordinary differential equations, which is called nowadays Picard-Vessiot Theory. Picard-Vessiot Theory is finite dimensional and the Galois group is a linear algebraic group. The first attempt of Galois theory of a general ordinary differential equations which is infinite dimensional, is done by the thesis of Drach [D]. He replaced an ordinary differential equation by a linear partial differential equation satisfied by the first integrals and looked for a Galois Theory of linear partial differential equations. It is widely admitted that the work of Drach is full of imcomplete definitions and gaps in proofs. In fact in a few months after Drach had got his degree, Vessiot was aware of the defects of Drach's thesis. Vessiot took the matter serious and devoted all his life to make the Drach theory complete. Vessiot got the grand prix of the academy of Paris in Mathematics in 1903 by a series of articles. However his theory is not written in a clear language in the modern sense

\footnotetext{
Received August 10, 1993.
} 
of the word so that it is difficult for us to understand what he wrote. So a general Galois Theory for ordinary differential equations ( $=$ an infinite dimensional differential Galois Theory) is not yet established at least in our taste.

Kolchin replaced a system of differential equations by a differential field extension. He introduced the notion of strongly normal extension of differential field and made finite dimensional differential Galois Theory complete and thus he generalized Picard-Vessiot Theory (cf. [K]). In the preface of [K1], he writes: Indeed, since as algebraic equation can be considered as a differential equation in which derivatives do not occur, it is possible to consider algebraic geometry as a special case of differential algebra. So the notion of strongly normal extension should generalize that of Galois extension. However we have unpleasant phenomena as follows:

(i) An extension $\mathbf{Q}(\sqrt{-1}) / \mathbf{Q}$ is Galois but not strongly normal;

(ii) A differential field extension $\mathbf{Q}(x, \exp x) / \mathbf{Q}(x$, exp $3 x)$ with derivation $d / d x$ is strongly normal but not Galois.

If there does not exist a natural definition unifying strongly normal extension and Galois extension, the existence of these examples contradicts the spirit of his words above. In this paper we do not touch general Galois Theory of ordinary differential equations but make Galois theory of differential equations satisfying the finiteness condition as transparent as possible. This is an inevitable task before we proceed to general Galois Theory. Whereas the Kolchin theory is formulated in the language of algebraic geometry of Weil working in a universal domain, theory of schemes seems more natural. In Weil's language it is not easy to treat algebraic varieties whose irreducible components are not absolutely irreducible over a field. This is the reason why Kolchin was obliged to adopt an awkward definition of algebraic group (cf. Chap. V, [K1]). We try to reduce the theory to a few principles. Namely Lemma (1.1) and a criterion in terms of Wronskian for functions to be linearly independent over the field of constants are basic and except for these two principles which belong to differential algebra, all the results are deduced by theory of schemes. In this attempt it becomes clear in what categories we have to work and what functors we have to consider. This process will contribute to clarify the problems in the general theory.

We introduce the notion of quasi-automorphic extension which unifies the both notions of strongly normal extension and Galois extension (Definition (2.5)). A differential field extension $L / K$ is strongly normal if and only if it is quasi-automorphic and the fields of constants of $L$ and $K$ coincide (Theorem (3.10)). So our notion explains well what strongly normal extension means. We 
prove that a differential field extension $L / K$ is quasi-automorphic if and only if (i) the constant field extension $C_{L} / C_{K}$ is finite and (ii) if there exist a model $X$ with derivation of $L / K$ and an algebraic group scheme $G$ over the field of constants $C_{K}$ such that $X$ is a principal homogeneous space of $G$ (Theorem (2.13)). In particular an abstract field extension $L / K$ of characteristic 0 is quasiautomorphic if and only if the extension is finite and $\operatorname{Spec} L$ is a principal homogeneous space of a finite group scheme over $K$ (Theorem (2.7)). The subtle point is to find a natural and correct definition of quasi-automorphic extension which excludes the function fields of arbitrary principal homogeneous spaces. We introduce an automorphic extension as a quasi-automorphic extension $L / K$ such that the morphism $\varphi_{1 A}^{*}: \mathbf{Q}\left[1 \otimes L, \phi_{h}^{*}(1 \otimes L)\right] \rightarrow A[[t]]\left[t^{-1}\right]$ is injective for every abstract field extension $A$ of the abstract field structure $K^{\natural}$ of the differential field $K$ (Definition (2.23)). We show that a classical Galois extension and a strong. ly normal extension are automorphic (Propositions (2.25) and (2.27)). There are automorphic extensions other than these two types. We prove that an almost classically Galois extension introduced by Greither and Pareigis [G] is automorphic (Corollary (2.26)).

Unfortunately in general for a quasi-automorphic extension $L / K$, the automorphy group is not uniquely determined (Examples (4.4), (4.9) and (4.15)) and we have not Galois correspondence (Remark (4.2)). However we have an injective map $\left\{C_{K}\right.$-algebraic subgroup schemes $\} \rightarrow$ differential intermediate fields of $\left.L / K\right\}$ (Proposition (4.5)). This result does not seem very interesting because it holds for the function field $K(V) / K$ of any principal homogeneous space $V$ over an abstract field $K$. For an automorphic extension we have the Galois correspondence. Namely the above injective map is in fact bijective if $L / K$ is automorphic (Theorem (4.10)). This result implies in particular that we have the Galois correspondence for an almost classically Galois extension which is one of the main results of $[G]$. Even for an automorphic extension the automorphic group is not uniquely deter mined (Example (4.15).

We always mean in this paper, by a Galois extension a finite Galois extension. Throughout the paper we assume that the characteristic of the fields is equal to 0 . However it seems that theory of Okugawa [O] fits well in our frame work. In that paper he treated Picard-Vessiot theory in char. $p>0$, where the correct generalization in char. $p>0$ of a differential ring is a ring with higher derivation. We can understand in view of the defintion of a quasi-automorphic extension why we have to introduce the higher derivation if we work in char. $p>0$ (Defintion (2.5)). It is an easy and pleasant task to sketch the proof of principal results of this paper in char. $p>0$. Yet there are subtle points treating eventually 
non-reduced schemes so that we will treat char. $p>0$ case in another paper. we are grateful to Mitsuhiro Takeuchi for teaching us what they are studying in the Galois theories of inseparable field extensions.

\section{§1. Preliminaries}

All the rings in this paper are assumed to be commutative and contain the field $\mathbf{Q}$ of rational numbers. A differential ring $(A, \delta)$ consists of a ring $A$ and a derivation $\delta: A \rightarrow A:$ Namely $\delta(a+b)=\delta a+\delta b, \delta(a b)=(\delta a) b+a(\delta b)$ for all $a, b \in A$. Kolchin calls such a ring an ordinary differential ring (cf. [K1] Chap. I, 1). We say that an element $a \in A$ is a constant if $\delta a=0$. Since $\delta 1=\delta(1.1)=$ $(\delta 1) 1+1(\delta 1)=2 \delta 1, \delta 1=0$ so that $\delta(\mathbf{Z})=0$ and hence $\delta(\mathbf{Q})=0$. So the ring $C_{A}$ of constants of $A$ is a $\mathbf{Q}$-algebra. We adopt the following usual notation: $a$ $=a^{(0)}, \delta a=a^{\prime}=a^{(1)}, \delta(\delta a)=a^{\prime \prime}=a^{(2)}$ and more generally $\delta a^{(n)}=a^{(n+1)}$ for $n$ $\geq 0$. We use the following convention: We denote the differential ring $(A, \delta)$ simply by $A$ if there is no danger of confusion for the choise of the derivation $\delta$. Forgetting the derivation $\delta$ of the differential ring $(A, \delta)$, we get an abstract ring $A$. When it is necessary to emphasize the abstract ring $A$, we denote it by $A^{\natural}$.

Let $f: A \rightarrow B$ be a homomorphism of differential rings, i.e. $f$ is a homomorphism of rings compatible with the derivations so that we can regard $f$ as a homomorphism $A^{\natural} \rightarrow B^{\natural}$ of abstract rings. We denote this homomorphism of abstract rings by $f^{\natural}: A^{\natural} \rightarrow B^{\natural}$. Thus we can define a forgetful functor ": (Diff-rng) $\rightarrow$ (Rng) of the category (Diff-rng) of differential rings to the category (Rng) of rings. The set of differential algebra homomorphisms will be denoted by $\operatorname{Diff-hom}(A, B)$ or simply by $\operatorname{Hom}(A, B)$ if there is no danger of confusing it with the set $\operatorname{Hom}\left(A^{\natural}, B^{\natural}\right)$ of abstract algebra homomorphisms.

Let $A$ be a differential ring, $B$ a differential subring of $A$ and $S$ a subset of $A$. According to Kolchin, we denote by $B\{S\}$ a differential subalgebra generated by $S$ over $B: B\{S\}$ is the smallest differential subalgebra of $A$ containing $B$ and $S$.

Let $L$ be a differential field, $M$ a differential subfield of $L$ and $S$ a subset of $A$. The differential subfield generated by $M$ and $S$ will be denoted by $M\langle S\rangle$. The following lemma is well-known but we give a proof due to Bialinicki-Birula because we use it in a basic way and because we had better understand the principle on which our theory depends.

Lemma (1.1) (Kolchin). Let $A$ be a differential ring and $K$ a differential subfield of $A$. Then the field $K$ and the ring $C_{A}$ of constants of $A$ are linearly disjoint over $C_{K}$. 
Proof. If the Lemma were false, then there would be $n$ elements $c_{1}, c_{2}, \ldots, c_{n}$ $\in C_{A}$ such that the $c_{i}$ are linearly independent over $C_{K}$ but are linearly dependent over $K$. We chose $n \geq 1$ minimum among such integers so that $n \geq 2$. Let

$$
\sum_{i=1}^{n} a_{i} c_{i}=0, \quad a_{\imath} \in K
$$

be a non-trivial linear relation so that at least one of the $a_{\imath}$ is not equal to 0 . We may assume $a_{n} \neq 0$. Then multiplying $a_{n}^{-1}$ to the relation (1.1.1), we may further assume $a_{n}=1$. So the relation (1.1.1) becomes

$$
a_{1} c_{1}+\cdots+a_{n-1} c_{n-1}+c_{n}=0 .
$$

We notice here at least one of the $a_{i}$ for $1 \leq i \leq n-1$ is not constant. Differentiating (1.1.2), we get $a_{1}^{\prime} c_{1}+\cdots+a_{n-1}^{\prime} c_{n-1}=0$, which is a non-trivial linear relation over $K$. So $c_{1}, c_{2}, \ldots, c_{n-1} \in C_{A}$ are linearly independent over $C_{K}$ but linearly dependent over $K$. This contradicts the choice of the integer $n$.

The proof of the lemma is simple but the lemma is fundamental and have applications.

Corollary (1.2). Let $L / K$ be a differential field extension such that $L$ is generated by constants over $K$. Then there is a 1:1 correspondence between the elements of the following two sets.

(i) The set $S$ of differential intermediate fields $L \supset M \supset K$.

(ii) The set $T$ of intermediate fields $C_{L} \supset D_{K} \supset C$.

Here we define a map $\Phi: S \rightarrow T$ by $M \mapsto C_{M}=M \cap C_{L}$ for $M \in S$ and a map $\Psi: T \rightarrow S$ by $D \mapsto D K$ (= a differential subfield of $L$ generated by $D$ and $K$ which coincides with a subfield of $L$ generated by $D$ and $K)$ for $D \in T$ so that $\Phi \circ \Psi=\operatorname{Id}_{T}$, $\Psi \circ \Phi=\operatorname{Id}_{S}$.

Proof. For $D \in T$, we have $K D \cap C_{L}=D$ so that $\Phi \circ \Psi=\operatorname{Id}_{T}$ since $C_{L}$ and $K$ are linearly disjoint over $C_{K}$. In fact the inclusion $K D \cap C_{L} \supset D$ being trivial, we have to show $K D \cap C_{L} \subset D$. Let $\left\{f_{\alpha}\right\}_{\alpha \in I}$ be a basis of a $C_{K}$-vector space $K$. If $c \in K D \cap C_{L} \subset K D$, then we can write $c=\sum_{\alpha \in I} a_{\alpha} f_{\alpha} / \sum_{\alpha \in I} b_{\alpha} f_{\alpha}$ with $a_{\alpha}, b_{\alpha} \in$ $D$, where at least one of the $b_{\alpha} \neq 0$ and the $a_{\alpha}, b_{\alpha}$ are equal to 0 except for a finite number of indices. So we have $\sum_{\alpha \in I} c b_{\alpha} f_{\alpha}=\sum_{\alpha \in I} a_{\alpha} f_{\alpha}$ and hence $\sum_{\alpha \in I}\left(c b_{\alpha}\right.$ $\left.-a_{\alpha}\right) f_{\alpha}=0$. Since the $c b_{\alpha}, a_{\alpha} \in C_{L}$, by the Lemma $c b_{\alpha}-a_{\alpha}=0$ for all $\alpha \in I$. Since there exists an index $\alpha \in I$ such that $b_{\alpha} \neq 0$, we get $c=a_{\alpha} / b_{\alpha}$ so that $c \in$ 
$D$. Now we have to show $\Psi \circ \Phi=I d_{S}$ or $K C_{M}=M$. Since the inclusion $K C_{M} \subset M$ is evident, it is sufficient to show $K C_{M} \supset M$. An element $u \in M$ is written as a quotient $u=\sum_{\alpha \in I} a_{\alpha} f_{\alpha} / \sum_{\alpha \in I} b_{\alpha} f_{\alpha}$ with $\sum_{\alpha \in I} b_{\alpha} f_{\alpha} \neq 0$, where the $a_{\alpha}, b_{\alpha} \in C_{L}$ are equal to 0 except for a finite number of indices. We get $u \sum_{\alpha \in I} a_{\alpha} f_{\alpha}=\sum_{\alpha \in I}$ $b_{\alpha} f_{\alpha}$ and hence $\sum_{\alpha \in I} a_{\alpha} u f_{\alpha}-\sum_{\alpha \in I} b_{\alpha} f_{\alpha}=0$. This shows that the $u f_{\alpha}$ and $f_{\alpha}$, which are elements of $M$, are linearly dependent over $C_{L}$. So it follows from the Lemma applied for $L$ and $M$ that the $u f_{\alpha}$ and $f_{\alpha}$ are linearly dependent over $C_{M}$ so that there exist $c_{\alpha}$ and $b_{\alpha} \in C_{M}$ for $\alpha \in I$ such that they are equal to 0 except for a finite number of indices and such that we have a non-trivial linear relation

$$
\sum_{\alpha \in I} c_{\alpha} u f_{\alpha}-\sum_{\alpha \in I} d_{\alpha} f_{\alpha}=0
$$

among the $u f_{\alpha}$ and $f_{\alpha}$. If all the $d_{\alpha}$ were equal to 0 , then $\sum_{\alpha \in I} c_{\alpha} u f_{\alpha}=0$ so that $\sum_{\alpha \in I} c_{\alpha} f_{\alpha}=0$. Since $\left\{f_{\alpha}\right\}_{\alpha \in I}$ is the basis of the $C_{K^{-}}$vector space $K$ and since $C_{L}$ and $K$ are linearly disjoint over $C_{K}$ by Lemma (1.1), all the $c_{\alpha}$ would be equal to 0 so that the linear relation (1.2.1) would be trivial. This contradicts the choice of the $c_{\alpha}$ and $d_{\alpha}$. Hence $\sum_{\alpha \in I} d_{\alpha} f_{\alpha} \neq 0$ so that $u=\sum_{\alpha \in I} c_{\alpha} f_{\alpha} / \sum_{\alpha \in I} d_{\alpha} f_{\alpha}$ and $u \in K C_{M}$. Thus the inclusion $K C_{M} \supset M$ is proved.

In the course of the proof of corollary, we proved the following result.

Corollary (1.3). Let $E / F$ be a differential field extension and $E \supset G \supset F$ a differential intermediate field. If there exists a set $W$ consisting of constants of $E$ such that $F(W) \supset G$, then $G$ is generated by constants over $F$ : Namely $G=F C_{G}$.

For a ring $B$, we denote by $B[[t]]$ the ring of formal power series with coefficients in $B$. The ring of formal Laurent series with coefficients in $B$ will be denoted by $B[[t]]\left[t^{-1}\right]$. These rings are differential rings with derivation $d / d t$. Let $\varphi: B \rightarrow C$ be a morphism of rings. Then it induces morphisms $B[[t]] \rightarrow$ $C[[t]], B[[t]]\left[t^{-1}\right] \rightarrow C[[t]]\left[t^{-1}\right]$ of differential rings. We denote the both homomorphisms by $\varphi[[t]]$ so that $\varphi[[t]]\left(\sum_{-\infty \ll n} a_{n} t^{n}\right)=\sum_{-\infty \ll n} \varphi\left(a_{n}\right) t^{n}$.

Let $A$ be a differential ring. A morphism $f: A \rightarrow B[[t]]$ of differential rings will be called a Tayloy morphism. Let us define a mapping $i: A \rightarrow A[[t]]$ by setting $i(a)=\sum \frac{\delta^{n} a}{n !} t^{n}$ for $a \in A$. Then $i$ is a morphism of differential rings or a Taylor morphism. We call $i$ the morphism of the universal Taylor expansion of the differential ring $A$ or the universal Taylor morphism for short. 
PROPOSITION (1.4). (i) The universal Taylor morphism is a monomorphism.

(ii) The universal Taylor morphism is universal among the Taylor morphisms. Namely let $f: A \rightarrow B[[t]]$ be a Taylor morphism. Then there exists a unique morphism $\varphi: A^{\natural}$ $\rightarrow B$ of rings such that $\varphi[[t]] \circ i=f$. In other words we have a bijection

$$
\Phi: \operatorname{Hom}\left(A^{\natural}, B\right) \rightarrow \operatorname{Diff}-\operatorname{hom}(A, B[[t]])
$$

sending a morphism $\varphi: A^{\natural} \rightarrow B$ of rings to a Taylor morphism $\varphi[[t]] \circ i: A \rightarrow$ $A^{\natural}[[t]] \rightarrow B[[t]]$.

Proof. Let $g: A^{\natural}[[t]] \rightarrow A^{\natural}$ be a morphism of taking the value at $t=0$ or the constant term of power series: $g(a(t))=a(0)$ for $a(t) \in A^{k}[[t]]$. Then $g \circ i$ $=\mathrm{Id}_{A}$, so that the universal Taylor morphism is a monomorphism. Let $h$ : $B[[t]] \rightarrow B$ be the morphism of taking the value at $t=0$. Then given $f \in$ $\operatorname{Diff-hom}(A, B[[t]])$, we get a morphism $h \circ f: A \rightarrow B[[t]] \rightarrow B$ of rings. Denoting $h \circ f$ by $\Psi(f)$, we get a map $\Psi: \operatorname{Diff-hom}(A, B[[t]]) \rightarrow \operatorname{Hom}\left(A^{\natural}, B\right)$. Since $\Psi \circ \Phi$ $=\mathrm{Id}, \Phi \circ \Psi=\mathrm{Id}$, Proposition is proved.

Let $L / K$ be an extension of differential field. We use the following condition very often

(F.C) The field $L$ is finitely generated over $K$ as an abstract field.

This condition will be called the finiteness condition.

The following lemma is well-known. We give a proof since we use it in an essential way and maybe algebraic geometer is not familiar with it.

Lemma (1.5). If a differential field extension $L / K$ satisfies the condition (F.C), then there exists a $K$-algebra $L_{0}$ finitely generated over $K$ such that $L_{0}$ is closed under the derivation $\delta$ and the quotient field of $L_{0}$ is $L$.

Proof. Let us observe the following: Let $S \subset L$ be a subset. For a subring $K[S]$ of $L$ to be closed under the derivation, it is necessary and sufficient that we have $s^{\prime} \in K[S]$ for any $s \in S$. Let $z_{1}, z_{2}, \ldots, z_{m} \in L$ be generators of the abstract field $L^{\natural}$ over $K^{\natural}$. Since the $\delta z_{i}$ are in $L$, we can find $f_{1}, f_{2}, \ldots, f_{m}, g_{1}, g_{2}$, $\ldots, g_{m} \in K^{\natural}\left[z_{1}, z_{2}, \ldots, z_{m}\right]$ with $g_{i} \neq 0$ such that $z_{i}^{\prime}=f_{i} / g_{i}$ for $1 \leq i \leq m$. We may assume that $g_{1}=g_{2}=\cdots=g_{m}$ by replacing the $g_{1}$ by $g_{1} g_{2} \cdots g_{m}$. Let us put $g=g_{1}=g_{2}=\cdots=g_{m}$ and $L_{0}=K\left[z_{1}, z_{2}, \cdots, z_{m}, 1 / g\right]$. We show that $L_{0}$ 
is closed under the derivation. We have $z_{i}^{\prime}=f_{\imath} / g \in L_{0}$ so that $f^{\prime} \in L_{0}$ for every $f \in K\left[z_{1}, z_{2}, \ldots, z_{m}\right]$. In particular $g^{\prime} \in L_{0}$ so that $(1 / g)^{\prime}=-g^{\prime} / g^{2} \in L_{0}$. Now Lemma follows from our observation above.

Let $(A, \delta)$ be a differential ring. The derivation $\delta: A \rightarrow A$ defines a derivation $A_{f} \rightarrow A_{f}$ for any $f \in A$ since $\delta\left[\frac{a}{f^{n}}\right]=\frac{a^{\prime} f^{n}-n a f^{n-1} f^{\prime}}{f^{2 n}}$. Since the structure sheaf $\hat{O}_{X}$ of a scheme $X=\operatorname{Spec} A$ is the sheaf of rings associated with a presheaf $D(f) \mapsto A_{f}$, we get a derivation $\delta: \mathscr{O}_{x} \rightarrow \mathscr{O}_{x}$. Here $D(f)=\left\{x \in \operatorname{Spec} A \mid f \in i_{x}\right.$ or $f(x) \neq 0\}$ in accordance with E.G.A. It is therefore convenient to adopt the following

Definition (1.6). A scheme with derivation consists of a scheme $X$ and a derivation $\delta: \mathfrak{O}_{X} \rightarrow \mathfrak{O}_{X}$. Namely $\Gamma\left(U, \mathfrak{O}_{X}\right)$ is a differential algebra with derivation $\delta$ for every open set $U$ of $X$ and the restriction morphism $\rho_{V}^{U}: \Gamma\left(U, \mathscr{O}_{X}\right) \rightarrow$ $\Gamma\left(V, \mathscr{O}_{X}\right)$ is a morphism of differential algebras for every pair of open sets $V \subset U$ of $X$. We denote the scheme with derivation by $(x, \delta)$ or simply by $X$ if there is no danger of confusion of the choice of the derivation. A morphism $f:\left(X, \delta_{1}\right) \rightarrow$ $\left(X, \delta_{2}\right)$ of schemes with derivation is a morphism of schemes $f: X \rightarrow Y$ commuting with the derivations. More precisely if we use the notation of E.G.A. Chap. I, $\S 1,2.3 .1$ so that the morphism $f$ consists of a continous map $\phi: X \rightarrow Y$ and a $\phi$-morphism $\theta: \mathscr{O}_{Y} \rightarrow \mathscr{O}_{X}$, then $\theta: \mathscr{O}_{Y} \rightarrow \mathscr{O}_{X}$ is a morphism of the sheaves of differential rings.

So we can speak of the category of schemes with derivation. Let $(A, \delta)$ be a differential ring. Then $\operatorname{Spec} A$ has a structure of a scheme with derivation and the differential ring $(A, \delta)$ is recovered from the scheme $\operatorname{Spec} A$ with derivation. Let $f: A \rightarrow B$ be a homomorphism of differential rings. The associated morphism with $f$ will be denoted by ${ }^{a} f: \operatorname{Spec} B \rightarrow \operatorname{Spec} A$. This is a morphism of schemes with derivation. It follows from E.G.A. Chap. I, Proposition (2.3.2) that we have a bijection $\operatorname{Diff-hom}(A, B) \simeq \operatorname{Diff-hom}(\operatorname{Spec} B, \operatorname{Spec} A), f \mapsto^{a} f$.

THEOREM (1.7). In the category of schemes with derivation, the fibre product exists.

Proof. Let $S$ be a scheme with derivation and $X, Y$ be two $S$-schemes with derivation. We show that the fibre product $X \times_{S} Y$ exists in the category of schemes with derivation. As in the case of usual schemes, we may assume that $X$, 
$Y, S$ are affine: There exist a differential algebra $C$ and differential $C$-algebras $A, B$ such that $X=\operatorname{Spec} A, Y=\operatorname{Spec} B$ and $S=\operatorname{Spec} C$. Then the theorem follows from the following

Proposition (1.8). Let $C$ be a differential ring and $A, B$ differential $C$-algebras. Then there exists a differential $C$-algebra $D$ with the following properties:

(i) There exist a differential C-algebra morphism $\varphi_{1}: A \mapsto D, \varphi_{2}: B \rightarrow D$;

(ii) For any differential $C$-algebra $E$ and differential $C$-morphisms $\phi_{1}: A \rightarrow E$, $\phi_{2}: B \rightarrow E$, there exists a unique differential $C$-morphism $f: D \rightarrow E$ such that $f \circ \varphi_{1}$ $=\phi_{1}, f \circ \varphi_{2}=\phi_{2}$ Namely we have a bijection

$$
\operatorname{Diff-hom}_{C}(D, E) \simeq \operatorname{Diff-hom}_{C}(A, E) \times \operatorname{Diff-hom}_{C}(B, E)
$$

sending $f \in \operatorname{Diff}_{-\operatorname{hom}_{C}}(D, E)$ to $\left(f \circ \varphi_{1}, f \circ \varphi_{2}\right)$

Proof. To be more precise let $\left(A, \delta_{1}\right)$ and $\left(B, \delta_{2}\right)$ be the differential $C$-algebras in question. We define a derivation $\delta$ on the tensor product $A$ " $\otimes_{C}$ " $B^{\natural}$ of the abstract algebras as follows: $\delta(a \otimes b)=\delta_{1}(a) \otimes b+a \otimes \delta_{2}(b)$ for $a$ $\in A$ and $b \in B$. We can check that $\delta$ is well-defined and is in fact a derivation on $A^{4} \bigotimes_{C}$, $B^{\natural}$. The canonical morphisms $\varphi_{1}: A \rightarrow A \otimes_{C} B, a \rightarrow a \otimes 1$ for $a \in A$ and $\varphi_{2}: B \rightarrow A \otimes_{C} B, b \rightarrow 1 \otimes b$ for $b \in B$ are $C$-morphisms of differential algebras. The morphisms $\varphi_{1}$ and $\varphi_{2}$ satisfies the condition (ii).

A rational map of a scheme $X$ to a scheme $Y$ is an equivalence class of morphisms of dense open sets of $X$ to $Y$. Two morphisms are considered to be equivalent if they coincide on a dense open sets (cf. E.G.A. Chap. I, Définition (8.1.2)). An $S$-rational map of an $S$-scheme to another $S$-scheme is a rational map which is an $S$-morphism. A rational function on $X$ is an $X$-rational map of $X$ to $X \otimes_{\mathbf{Z}} \mathbf{Z}[T]$, where $T$ is a variable over $\mathbf{Z}$. All the rational functions on $X$ form a ring $R(X)$. If $X$ irreducible, then the ring $R(X)$ coincides with the local ring $\mathscr{O}_{x}$ at the generic point $x \in X$. Moreover if $X$ is reduced, then $R(X)=\mathscr{O}_{x}$ is a field (cf. E.G.A. Chap. I, Proposition (8.1.5)).

Let $L / K$ be a differential field extension satisfying the condition (F.C) and $L_{0}$ be the differential ring of Lemma (1.5). So Spec $L_{0}$ is a scheme with derivation over the scheme $\operatorname{Spec} K$ with derivation.

Definition (1.9). A model of the differential field extension $L / K$ is a $K$-scheme $X$ with derivation such that the underlying scheme $X^{\natural}$ is reduced, irreducible and of finite type over $K^{\natural}$ and such that the field $K(X)$ of rational functions of $X$ is $K$-isomorphic to $L$ as a differential field. 
Remarks (1.9.1). If $X$ is a model of $L / K$, then there exist two non-empty open sets $U \subset X, V \subset \operatorname{Spec} L_{0}$ and a $K$-morphism $f: U \rightarrow \operatorname{Spec} L_{0}, g: V \rightarrow X$ of schemes with derivation such that $f \circ g=\mathrm{Id}, g \circ f=\mathrm{Id}$. Since these open sets are universally $K$-scheme theoretically dense respectively in Spec $L_{0}$ and in $X$, we can say that there exists a $K$-pseudo-isomorphism $X \cdots \rightarrow$ Spec $L_{0}$ compatible with derivation. (We recall below the definition of a pseudo-morphism.) This formulation is not only formal but also useful in our theory since we study not only models over $K$ but also their various base changes for morphisms $S \rightarrow$ Spec $K$, where the scheme $S$ with derivation is not necessarily reduced.

(1.9.2) A germ of introducing schemes with derivation appeared already in the 19-th century when the mathematicians of that time studied a condition for an ordinary differential equation of the first order to be free from movable singular points.

(1.9.3) In general, we can not find a complete model with derivation of a differential field extension $L / K: A$ model $X$ with derivation whose underlying scheme is proper over $K$. For an ordinary algebraic differential equation of the first order or for a differential field extension $L / K$ satisfying the finiteness condition (CD) with $\operatorname{tr} . \mathrm{d}[L: K]=1$, the complete model with derivation exists if and only if the equation is free from the movable singlular points.

We can show that if $y$ satisfies the Painleve equation $y^{\prime \prime}=6 y^{2}+x$, there is no complete model of a differential field extension $\mathbf{C}(x)\langle y\rangle / \boldsymbol{C}(x)$, where the derivation is $d / d x$.

(1.9.4) After we had done our work, we learned that in recent monograph $[\mathrm{Bu}]$, the scheme with derivation plays an important role.

Let us recall the notion of pseudo-morphism of E.G.A. Chap. IV, §20. Let $X$ be a scheme. An open set $U$ of $X$ is scheme theoretically dense by defintion if the restriction map $\Gamma\left(V, \mathscr{O}_{X}\right) \rightarrow \Gamma\left(V \cap U, \mathscr{O}_{X}\right)$ is a monomorphism for every open set $V$ of $X$ (cf. E.G.A. Chap. IV, (20.2.1)). When $X$ is an $S$-scheme, we say that an open set $U$ of $X$ is universally scheme theoretically dense over $S$ or universally $S$-scheme theoretically dense if for every morphism $S^{\prime} \rightarrow S$ of schemes, the base change $U_{S}$, is scheme theoretically dense in $X_{S}$, Let $Y$ be another $S$-scheme. An $S$-pseudo-morphism of $X$ to $Y$ is an $S$-morphism $f: U \rightarrow Y$ of $S$-schemes where $U$ is an open set of $X$ scheme theoretically dense over $S$ modulo the following equivalence relation: We identify two such morphisms $f: U \rightarrow Y$ and $f^{\prime}: U^{\prime} \rightarrow Y$ if they coincide on the intersection $U \cap U^{\prime}$. We say that the couple $(U, f)$ represents its equivalence class.

We define a pseudo-function on a scheme $X$ as we introduced a rational func- 
tion on $X$. Namely a pseudo-function is an $X$-pseudo-morphism of $X$ to $X \otimes_{\mathbf{Z}} \mathbf{Z}[T]$, where $T$ is a variable over $\mathbf{Z}$. The notion of pseudo-function is finer and hence more useful than that of rational function. In our theory we should deal with pseudo-functions rather than rational functions as natural objects because as we said above, we have to study the base changes $X \otimes S / S$ of a model $X$ which is a $K$-scheme with derivation. But in our analysis only rational functions appear and pseudo-functions are hidded by virtue of the following result (E.G.A., Chap. IV, Remarque (20.2.9)).

If $X$ is a reduced scheme, then the following conditions on an open set $U$ of $X$ are equivalent:

(i) Then open set $U$ is dense;

(ii) Then open set $U$ is scheme theoretically dense.

So the notion of pseudo-morphism (resp. pseudo-function) on a reduced scheme coincides with that of rational map (resp. rational function).

A morphism $f: X \rightarrow Y$ of schemes is scheme theoretically dominant if the morphism $\Gamma\left(U, \mathscr{O}_{Y}\right) \rightarrow \Gamma\left(f^{-1}(U), \mathscr{O}_{X}\right)$ is a monomorphism for every open set $U$ of $Y$. Let $f: X \rightarrow Y$ be morphism of schemes. We say that the morphism $f$ is universally scheme theoretically dominant if the base change $f_{S^{\prime}}: X_{S^{\prime}} \rightarrow Y_{S^{\prime}}$ is scheme theoretically dominant for every morphism $S^{\prime} \rightarrow S$ of schemes.

Let us notice the follwing fact. Let $f: Y \rightarrow Z$ be a $k$-morphism of algebraic schemes over a field $k$ such that $Z$ is irreducible and reduced. Then the morphism $f: Y \rightarrow Z$ is universally scheme theoretically dominant over $k$ if and only if the image $f(Y)$ contains the genbric point of $Z$.

We say that an $S$-pseudo-morphism $f: X \cdots \rightarrow Y$ is universally scheme theoretically dominant if it is represented by a couple $(f, U)$ such that the $S$-morphism $f: U \rightarrow X$ is universally scheme theoretically dominant over $S$. Let $Z$ be a third $S$-scheme and $g: Y \longrightarrow Z$ be an $S$-pseudo-morphism. According to E.G.A. Chap. IV, (20.3.2) if the $S$-pseudo-morphism $f: X \cdots \rightarrow Y$ is universally scheme theoretically dominant over $S$, then we can define an $S$-pseudo-morphism $g \circ f: X \rightarrow Z$. We say that a $S$-pseudo-morphism $f: X \rightarrow Y$ is an $S$-pseudoisomorphism if $f$ is universally scheme theoretically dominant over $S$ and there exists an $S$-pseudo-morphism $g: Y \rightarrow \rightarrow X$ such that $g$ is universally scheme theoretically dominant over $S$ with $g \circ f=\operatorname{Id}_{X}, f \circ g=\operatorname{Id}_{Y}$. We denote by Ps.aut $X$ the group of $S$-pseudo-automorphisms of $X$, i.e. the group of $S$-pseudo-isomorphisms of $X$ to $X$. If we set

$$
\text { Ps.aut }{ }_{S} X\left(S^{\prime}\right)=\text { Ps.aut }_{S^{\prime}}\left(X \times_{S} S^{\prime}\right),
$$

then Ps.aut: $(\mathrm{Sch} / S)^{0} \rightarrow(\mathrm{Grp})$ is a group functor. The notation Ps.aut ${ }_{S} X$, 
Ps.aut ${ }_{S} X$ is due to E.G.A. but it seems that most authors denote the group Ps.aut $X$ by $\operatorname{Bir}_{S} X$ when $X$ is an algebraic variety over a field $k$ so that $S=$ Spec $k$. Hence we denote the group $\operatorname{Ps}_{\text {saut }} X$ by $\operatorname{Bir}_{k} X$ and the group functor Ps.aut $X$ by $\mathbf{B i r}_{S} X$ when $S$ is the spectrum of a field.

Let us assume that $k$ is a field and $S=\operatorname{Spec} k$. Let $F / k$ is a field extension finitely generated over $k$. A reduced algebraic $k$-scheme $X$ whose ring of meromorphic functions ( $=$ rational functions) is $k$-isomorphic $F$ will be called a model of $F / k$. Let $X, X^{\prime}$ be models of $F / k$. Then there exists a $k$-pseudo-isomorphism $X \cdots \rightarrow X^{\prime}$ so that the group $\mathbf{B i r}_{S} X\left(S^{\prime}\right)$ is isomorphic to the $\operatorname{group} \operatorname{Bir}_{k} X^{\prime}\left(S^{\prime}\right)$ for any $k$-scheme $S^{\prime}$. So the group functor $\mathbf{B i r}_{S} X$ is isomorphic to the group functor $\mathbf{B i r}_{k} X^{\prime}$. In other words the group $\mathbf{B i r}_{k} X(S)$ and the group functor $\mathbf{B i r}_{k} X$ are independent of the choice of model $X$. So we denote $\mathbf{B i r}_{k} X$ by $\mathbf{B i r}_{k} F$.

Proposition (1.10) (Demazure [D], 1, Proposition 1). Let $X, Y$ be schemes smooth and of finite type over a scheme $S$. The following conditions for an $S$-pseudomorphism $f X \rightarrow Y$ are equivalent.

(i) $f$ is an $S$-pseudo-isomorphism.

(ii) There exist universally $S$-scheme theoretically dense open sets $U$ of $X$ and $V$ of $Y$ such that $f$ induces an $S$-isomorphism $U \rightarrow V$.

Proposition (1.11). Let $L / K$ be a differential field extension with $C_{L}=C_{K}=$ $C$ satisfying the finiteness condition (F.C). Let $X$ be a model with derivation of $L / K$, $A$ a $C_{K}$-algebra and $U$ a universally scheme theoretically dense open set of $X \otimes_{C} A$. If $f \in \Gamma\left(U, \mathscr{O}_{X \otimes_{C} A}\right)$ is constant, then $f$ comes from a global section of $\mathscr{O}_{\mathrm{Spec} A}$ : There exists an element $g \in \Gamma\left(\operatorname{Spec} A, \mathscr{O}_{\mathrm{Spec} A}\right)=A$ such that $g \circ p=f$, where $p$ denotes the projection $U \subset X \otimes_{C} A \rightarrow \operatorname{Spec} A$.

Remark. A morphism $Y \rightarrow Z$ of schemes consists of a of a continuous map $g: Y \rightarrow Z$ of the underlying topological spaces $Y, Z$ and of a morphism $\mathfrak{O}_{Z} \rightarrow \mathscr{O}_{Y}$ of sheaves of rings (cf. E.G.A. Chap. I, 2.3). So in the Proposition we mean by the notation $g \circ p$ the image of the section $g$ of the structure sheaf $\mathscr{O}_{A}$ by the projection morphism $p: U \rightarrow \operatorname{Spec} A$.

Proof. Since $C=C_{K}=C_{L}$ is algebraically closed in $L, X_{B}$ is irreducible for any field extension $B / C$. In particular for any point $z \in \operatorname{Spec} A$, the fibre $p^{-1}(z)=X \otimes_{C} C(z)$ is irreducible. Therefore if $W$ is an open set of $X_{A}$ such that $W_{z}=W \otimes_{C} C(z)$ is not empty, then $W_{z}$ is dense in $X \otimes_{C} C(z)$. Since $X_{A} \rightarrow$ $\operatorname{Spec} A$ is faithfully flat, it follows from the above investigation that we have to 
prove the following assertion:

(1.11.1) For any ponit $x \in U$, there exists a neighbourhood $V$ of $x$ depending on $x$ such that the restriction $\rho_{V}^{U}(f)$ of $f$ comes from a section of the structure sheaf $\mathscr{O}_{\text {Spec } A}$ over $p(V)$. Here we notice that since $p: X_{A} \rightarrow \operatorname{Spec} A$ is flat, $p$ is an open map and hence $p(V)$ is an open sub-set of $\operatorname{Spec} A$. Let $x$ be a point of $U$. We can find a neighbourhood $D(h)$ of $x$ in $U$ with $h \in \Gamma\left(X_{A}, \mathscr{O}_{X_{A}}\right)$ so that

$$
\rho_{D(h)}^{U}(f)=k / h
$$

on $D(h)$ with $k \in \Gamma\left(X_{A}, \mathcal{O}_{X_{A}}\right)$. This is an identity in $\Gamma\left(D(h), \mathscr{O}_{X_{A}}\right)=\left(L^{0} \otimes_{C} A\right)_{h}$. Let us set $R=\Gamma\left(D(h), \mathscr{O}_{X_{A}}\right)$, then the ring $C_{R}$ of constants and $L^{0}$ are linearly disjoint over $C$ by Lemma (1.1).

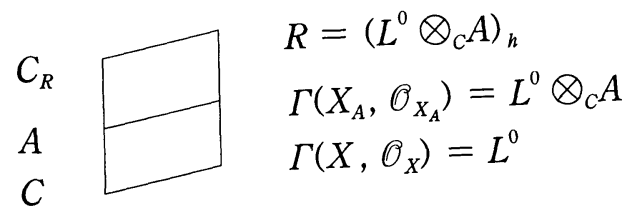

Let $\left\{e_{i}\right\}_{i \in I}$ be a basis of the $C$-vector space $L^{0}$. We can write $k=\sum_{i} e_{i} \otimes b_{i}$, $h=\sum_{\imath} e_{i} \otimes c_{i}$ with $b_{i}, c_{i} \in A$, where the $b_{\imath}$ and the $c_{i}$ are zero except for a finite number of indices. Setting $\bar{f}=\rho_{D(h)}^{U}(f)$, we get by (1.11.2) $\bar{f}=k / h$ in $\Gamma\left(x_{A}, \mathscr{O}_{X_{A}}\right)_{h}$. Therefore $\bar{f} h=k$ in $\Gamma\left(X_{A}, \mathscr{O}_{X_{A}}\right)_{h}$ and hence $\sum_{i}\left(e_{i} \otimes 1\right) \bar{f}\left(1 \otimes c_{i}\right)=\bar{f}\left(\sum_{\imath} e_{\imath} \otimes c_{\imath}\right)$ $=\sum_{\imath} e_{i} \otimes b_{\imath}=\sum_{\imath}\left(e_{i} \otimes 1\right)\left(1 \otimes b_{\imath}\right)$. Namely we have $\sum_{\imath}\left(e_{i} \otimes 1\right)\left(\bar{f}\left(1 \otimes c_{i}\right)\right.$ $\left.-1 \otimes b_{i}\right)=0$. Since the $\bar{f}\left(1 \otimes c_{i}\right)-1 \otimes b_{i}$ are in $C_{R}$ and the $e_{i} \otimes 1$ are $C_{R}$-linearly independent, we conclude $\bar{f}\left(1 \otimes c_{\imath}\right)=1 \otimes b_{\imath}$ for all $i \in I$. Since $0 \neq$ $h(x)=\sum_{\imath} e_{i}(x) c_{\imath}(x)$, there exists an index $i$ such that $c_{i}(x) \neq 0$. Namely in a neighbourhood $V$ of $x$, we have $\rho_{V}^{U}(f)=b_{i} / c_{i}$.

Lemma (1.12). Let $R$ be a differential ring with 1 . If $R$ is a direct product of a finite number of ideals $R_{i}: R=\prod_{i=1}^{n} R_{i}$, then the ideals $R_{i}$ are closed under the derivation so that $R$ is a direct product of the differential ideals $R_{i}$.

Proof. Let $1=e_{1}+e_{2}+\cdots+e_{n}$ be the orthogonal idempotent decomposition of 1 so that $R_{i}=R e_{i}$ for $1 \leq i \leq n$. Since $e_{i} e_{j}=0$ if $i \neq j, 0=\left(e_{i} e_{j}\right)^{\prime}=e_{i}^{\prime} e_{j}$ $+e_{i} e_{j}^{\prime}$ and $e_{i}^{\prime} e_{j}=-e_{i} e_{j}^{\prime} \in R_{i} \cap R_{j}=0$. Thus the $j$-th component of $e_{i}^{\prime}=0$ for $i \neq j$ and $e_{i}^{\prime} \in R_{i}$ so that the ring $R_{\imath}=R e_{i}$ is closed under the derivation. In fact 
$\left(a e_{\imath}\right)^{\prime}=a^{\prime} e_{i}+a e_{\imath}^{\prime} \in R_{\imath}$ since the $R_{\imath}$ are ideals of $R$.

COROLlary (1.12.1). We using the notation of Lemma (1.12) and its proof, the idempotents $e_{i}$ are constants for $1 \leq i \leq n$. If we set $C_{i}=C_{R_{i}}$ for $1 \leq i \leq n$, then the ring $C_{R}$ of constants of $R$ is a direct product of the $C_{i}: C_{R}=\prod_{i=1}^{n} C_{i}$.

Proof. Differentiating the idempotent decomposition $1=e_{1}+e_{2}+\cdots+e_{n}$, we get $0=e_{1}^{\prime}+e_{2}^{\prime}+\cdots+e_{n}^{\prime}$ so that $e_{i}^{\prime}=0$ for $1 \leq i \leq n$ since $e_{i}^{\prime} \in R_{i}$ by Lemma (1.12). Thus the first assertion is proved. As for the second, it is sufficient to show that every constant $c$ of $R$ can be written as a sum of an element of the $C_{i}$ $1 \leq i \leq n$. In fact $c=c e_{1}+c e_{2}+\cdots+c e_{n}$ is the decomposition of the element $c$. As we have just seen, the $e_{i}$ are constants so that $c e_{i} \in C_{i}$ so that the second assertion is proved.

Most of schemes $X$ which arise in this paper are reduced algebraic schemes over a field $k$ and we study their base changes $X_{S}$ for a $k$-schemes $S$. Let us recall that a $k$-algebraic scheme is a scheme of finite type over field $k$ (cf. E.G.A. Chap. I, 6.5.1). We have to consider however some exceptions such as Spec $k[[t]]\left[t^{-1}\right]$.

Let $L / K$ be a differential field extension satisfying the finiteness condition (F.C). Since we are in characteristic 0 , the tensor product $L \bigotimes_{K} L$ is reduced and the total quotient ring $\mathbf{Q}\left(L \otimes_{K} L\right)$ is a direct product of the ideals $M_{i}: \mathbf{Q}(L$ $\left.\bigotimes_{K} L\right)=\Pi_{\imath=1}^{n} M_{i}$ and the $M_{\imath}$ are fields (see for example [Z.S] Chap. IV, §3). Then by Lemma (1.12) the $M_{\imath}$ are differential fields.

Geometrically let $X$ be a model of $L / K$. Since $X$ is noetherian and we are in characteristic $0, X \otimes_{K} X$ is noetherian and reduced. There are finitely many irreducible components $Y_{\imath}$ of $X \times_{K} X: X \times_{K} X=\cup_{i=1}^{n} Y_{\imath}$. The total quotient ring $\mathbf{Q}\left(L_{0} \otimes_{K} L_{0}\right)$ which coincides with $\mathbf{Q}\left(L \bigotimes_{K} L\right)$ is isomorphic to the direct product of the $K\left(Y_{i}\right)=M_{i}$.

Anyhow we have the projections $f_{i}: \mathbf{Q}\left(L \otimes_{K} L\right) \rightarrow M_{\imath}, 1 \leq i \leq n$ and two $K$-morphisms $\varphi_{1}, \varphi_{2}: L \rightarrow L \otimes_{K} L$ such that $\varphi_{1}$ identifies $L$ with $L \otimes 1 \subset \mathbf{Q}(L$ $\left.\bigotimes_{K} L\right)$ and $\varphi_{2}$ identifies $L$ with $1 \otimes L \subset \mathbf{Q}\left(L \bigotimes_{K} L\right)$. We get two $K$-morphisms $f_{i}^{1}, f_{i}^{2}: L \rightarrow M_{i}$ by setting $f_{i}^{1}=f_{i} \circ \varphi_{1}, f_{i}^{2}=f_{i} \circ \varphi_{2}$. We set for further purpose $f_{i}^{1}(L)=L_{i}^{(1)}, f_{i}^{2}(L)=L_{i}^{(2)}$ for $1 \leq i \leq n$.

Let $\mathscr{C}$ be a category and $\mathrm{X}$ an object of $\mathscr{C}$. Then $h_{X}: \mathscr{C}^{0} \rightarrow$ (Set) sending $Y$ to $h_{X}(Y)=\operatorname{Hom}(Y, X)$ is a functor $\left(\mathscr{C}^{0}\right.$ being the dual category of $\left.C\right)$. We some- 
times denote $h_{X}(Y)$ by $X(Y)$. Let $S=\operatorname{Spec} A$ be an affine scheme and $\mathscr{C}$ a category of $S$-schemes. We often denote $X(\operatorname{Spec} B)$ by $X(B)$ for an $A$-algebra $B$.

Let $X_{i}, 1 \leq i \leq n$ be objects of a category $\mathscr{C}$ such that the product $X=$ $\Pi_{i=1}^{n} X_{i}$ exists in $\mathscr{C}$. The $i$-th projection $X \rightarrow X_{i}$ will be denoted by $p_{i}$ for $1 \leq i$ $\leq n$.

Let $u$ s recall the definition of principal homogeneous space. An operation $(G, X)$ of a group $G$ on a set $X$ is a principal homogeneous space if the following condition is satisfied: If $x \in X$, then a map $G \rightarrow X$ sending $g \in G$ to $g x \in X$ is bijective. It is convenient to interpret that $(G, \phi)$ is a principal homogeneous space for any group $G$. We often say that the set $X$ is a principal homogeneous space of the group $G$ without making the operation $G \times X \rightarrow X$ precise. Let $F: \mathscr{C}$ $\rightarrow$ (Set) be a functor and $G: \mathscr{C} \rightarrow(\mathrm{Grp})$ be a group functor. If the functor $G$ operates on the functor $F$ in such a way that $(G(S), F(S))$ is a principal homogeneous space for every object $S \in$ ob $\mathscr{C}$, we say that $(G, F)$ is a principal homogeneous space or $F$ is principal homogeneous space of $G$.

Let $S$ be a scheme and $\mathscr{C}$ the dual of the category of $S$-schemes. Let $G$ an $S$-group scheme and $X$ an $S$-scheme such that $G$ operates on $X:$ We have an $S$-morphism $\mu: G \times X \rightarrow X$ making the several well-known diagrams commutaitve. We can regard $G$ and $X$ as functors on the category $\mathscr{C}$. Then $(G, X)$ is a principal homogeneous space if and only if the morphism $\left(\mu, p_{2}\right): G \times{ }_{S} X \rightarrow X$ $\times_{S} X$ is an isomorphism.

Let $G$ be an $S$-group scheme and $X$ an $S$-scheme such that $G$ operates on $X$ over $S$. We say that the operation $(G, X)$ is generically transitive if the morphism $\left(\mu, p_{2}\right): G \times{ }_{S} X \rightarrow X \times_{S} X,(g, x) \rightarrow(g x, x)$ is scheme theoretically dominant. Let us recall that a morphism $h: V \rightarrow W$ of schemes is scheme theoretically dominant by definition if the morphism $\Gamma\left(U, \mathscr{O}_{V}\right) \rightarrow \Gamma\left(h^{-1}(U), \mathscr{O}_{V}\right)$ is injective for every open set $U$ of $V$.

The following result which we learned from Miyanishi seems well-known.

Lemma (1.13). Let $k$ be a field of characteristic 0 and $G$ a $k$-algebraic group scheme. Then the irreducible component $G_{0}$ of $G$ containing 1 is absolutely irreducible: The base change $G_{0 K}$ remains irreducible for any field extension $K / k$

Proof. We have to show that the extension $k\left(G_{0}\right) / k$ is regular. Since we are in characteristic 0 , it is sufficient to show that $k$ is algebraically closed in $k\left(G_{0}\right)$. Let $f \in k\left(G_{0}\right)$ be algebraic over $k$ and let $f^{n}+a_{1} f^{n-1}+\cdots+a_{n}=0, a_{i} \in$ $k(1 \leq i \leq n)$ be the minimal polynomial of $f$ over $k$. Since $G_{0}$ is reduced, $G_{0}$ is 
smooth over $k$ so that the local ring $\mathscr{O}_{G_{0} g}$ is regular for any point $g \in G_{0}$. Thus $\mathscr{O}_{G_{0} g}$ is a unique factorization domain and hence normal so that $f \in \mathscr{O}_{G_{0} g}$. In particular for the point $1 \in G, f \in \mathscr{O}_{G_{0}}$. Since 1 is a $k$-rational point of $G_{0}$, we have a $k$-morphism $\mathscr{O}_{G_{0} 1} \rightarrow k$ so that we have $\bar{f}^{n}+a_{1} \bar{f}^{n-1}+\cdots+a_{n}=0 \bar{f}$ being the image of $f$ by the $k$-morphism $\mathscr{O}_{G_{0} 1} \rightarrow k$. Hence $\bar{f} \in k$ is a root of the irreducible polynomial $x^{n}+a_{1} x^{n-1}+\cdots+a_{n} \in k[x]$ so that $n=1$ and $f=-a_{1} \in k$. We have thus proved that $k$ is algebraically closed in $k\left(G_{0}\right)$. Hence the Lemma is proved.

In our papers [U1], [U2] we defined algebraic group germ as an analogue of analytic group germ which is formulated in Bourbaki [Bo]. Roughly speaking a group germ is a local group law consisting of a local composition law, a local in verse and a local unit. In algebraic geometry, however rational group law which was first introduced by Weil [W], seems to arise more naturally than algebraic group germ.

Definition (1.14). A rational group law over a field $k$ (of characteristic 0) consists of a reduced $k$-algebraic scheme $Z$, a $k$-rational map $m: Z \times{ }_{k} Z \cdots \rightarrow Z$ satisfying the following conditions:

(i) The map $\left(m, p_{2}\right): Z \times{ }_{k} Z \cdots \rightarrow Z \times{ }_{k} Z$ is dominant;

(ii) The following diagram is commutative;

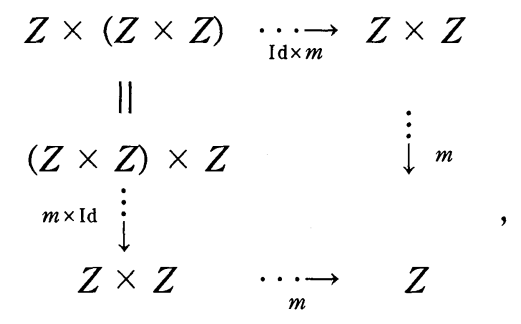

where the products are taken over $k$.

Remark (1.14.1). If the field $k$ is of characteristic $p>0$, then $X \times_{k} X$ is not necessarily reduced so that the condition (1.14.i) should be modified.

Proposition (1.15). Let $Z$ be a rational group law. Then there exists a $k$ algebraic scheme $G$ birationally equivalent to $X$ : i.e. There exists a $k$-birational map $G \cdots \rightarrow X$ compatible with group laws. 
When $X$ is absolutely irreducible over $k$, Proposition is due to weil [W]. An extreme generalization of Weil's result is proved in S.G.A.D., Exposé XVIII. Let us notice that the algebraic group scheme $G$ is uniquely determined up to isomorphisms.

The same remark should be given for law chunk of algebraic operation introduced in [U1].

Definition (1.16). Let $G$ be an algebraic group scheme over a field $k$ of characteristic 0 and $X$ a reduced $k$-algebraic scheme. We say that a $k$-rational map $\mu: G \times{ }_{k} X \cdots \rightarrow X$ is a rational operation of the algebraic group $G$ on the algebraic scheme $X$ if the following conditions are satisfied:

(i) A rational map $\left(p_{1}, \mu\right): G \times_{k} X \cdots \rightarrow G \times_{k} X,(g, x) \mapsto(g, \mu(g, x))$ is dominant;

(ii) The diagram

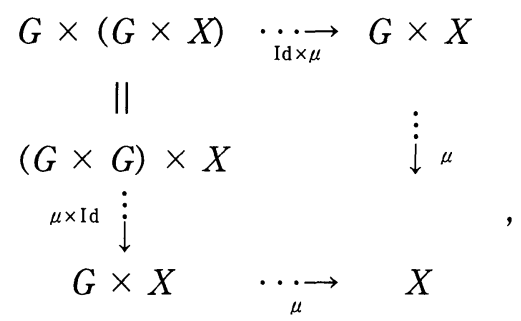

is commutative, where the products are taken over $k$.

Proposition (1.17). Let $k$ be a field (of char. 0). For a rational operation $(G, X)$ of a $k$-group scheme $G$ on a reduced $k$-algebraic scheme $X$, there exists an operation $(G, Y)$ over $k$ which is birationally equivalent to $(G, X)$.

Proof. When all the irreducible components of $G$ and $X$ are absolutely irreducible, the Proposition is proved Weil [W]. Rosenlicht proved the Proposition under an additional hypothesis that the variety $X$ and the irreducible components of $G$ are absolutely irreducible (cf. [R] Theorem 1). His proof works if all the irreducible components of the scheme $X$ and those of $G$ are absolutely irreducible. Now by Galois descent the Proposition follows from

Lemma (1.18). Keeping the notation of Proposition (1.17), we assume furthermore that $k$ is algebraically closed. (i) There exists an operation $(G, Y)$ birationally equivalent to $(G, X)$ such that $Y$ is quasi-projective. (ii) Let $\left(G, Y_{1}\right),\left(G, Y_{2}\right)$ be operations 
birationally equivalent to $(G, X)$. Then there exist $G$-invariant dense open sets $U_{1}$, $U_{2}$ such that $\left(G, U_{1}\right)$ is isomorphic to $\left(G, U_{2}\right)$.

Proof. The second assertion is proved following the Proof of Corollary to Theorem $1[\mathrm{R}]$. There the case where $X$ is absolutely irreducible is treated. It remains to show the first assertion. As we noticed above, there exists an operation $(G, Z)$ birationally equivalent to $(G, X)$. We show that there exists a variety of orbits of $(G, Z)$ by Theorem $2[\mathrm{R}]$. There exists a $G$-invariant dense open set $U$ of $Z$ and a morphism $\tau: U \rightarrow W$ of of reduced $k$-algebraic schemes such that a fibre $U_{\xi}$ is a $G_{k(\xi)}$-homogeneous space for any point $\xi \in W$. Let us first assume that $W$ is irreducible. Let $\eta: \operatorname{Spec} k(W) \rightarrow W$ be the generic point so that $U_{\eta}$ is the generic fibre. $\left(G_{k(W)}, U_{\eta}\right)$ is a homogeneous space and hence $U_{\eta}$ is quasiprojective as is well known. Therefore there exists a non-empty affine open set $V$ such that the morphism $\tau$ is quasi-projective when restricted over $\tau^{-1}(V) \rightarrow V$. So $\tau^{-1} V$ is quasi-projective since $V$ is affine. In general if $W$ is not irreducibe, we can argue at the generic points of irreducible components of $W$ to conclude that there exists a $G$-invariant dense open subset $U$ of $Z$ which is quasi-projective.

\section{§2. Automorphic extensions}

Let $L / K$ be a differential field extension satisfying the finiteness condition (F. C) of $\S 1$. Let $X$ be a model with derivation of the extension $L / K$. We sometimes denote by $C$ the field $C_{K}$ of constants of $K$ to simplify the notation. The category of $C$-schemes will be denoted by $\left(\mathrm{Sch} / C\right.$ ). We define a group functor $\mathbf{D i f f}^{-\mathbf{b i r}}{ }_{K} L$ : $(\mathrm{Sch} / C)^{0} \rightarrow(\mathrm{Grp})$ by setting

$$
\begin{aligned}
\text { Diff- } \operatorname{bir}_{K} L(S)= & \left\{f \in \text { Ps.aut } X \times{ }_{C} S / \operatorname{Spec} K \times_{C} S \mid\right. \\
& \text { pseudo-morphism } f \text { is compatible with derivation }\} .
\end{aligned}
$$

In fact if $\mathbf{D i f f}-\mathbf{b i r}_{K} L(S)$, then $\varphi^{*} f \in \mathbf{D i f f}_{-} \mathbf{b i r}_{K} L\left(S^{\prime}\right)$ for a morphism $\varphi: S^{\prime}$ $\rightarrow S$ of $C$-schemes so that $\mathbf{D i f f}_{-} \mathbf{b i r}_{K} L$ is a group functor. It is evident that the definition is independent of the choice of model.

Example (2.1). We consider a differential field $\left(\mathbf{C}[[x]]\left[x^{-1}\right], d / d x\right)$ of the formal Laurent series with coefficients in the complex numer field $\mathbf{C}$. Let us set $y=\exp (x)=\sum_{n=0}^{\infty} \frac{1}{n !} x^{n} \in \mathbf{C}[[x]]\left[x^{-1}\right]$. The series $y$ satisfies a differential equation 


$$
d y / d x=y
$$

We also set $K=\mathbf{C}(x)$ so that $K$ is a differential subfield of $\mathbf{C}[[x]]\left[x^{-1}\right]$, By the differential equation (2.1.1), $L=K(y)$ is a differential subfield of $\mathbf{C}[[x]]\left[x^{-1}\right]$ by considering the Laurent expansion at $x=0$ of rational functions. So we obtained a differential field extension $L / K$. Since these fields are subfields of $\mathbf{C}[[x]]\left[x^{-1}\right]$, $C_{L}=C_{K}=\mathbf{C}$. Let us take $X=\operatorname{Spec} K\left[y, y^{-1}\right]$ as a model of $L / K$. Let us determine Diff-bir ${ }_{K} L(\operatorname{Spec} A)$ for a $\mathbf{C}$-algebra $A$. Let $f \in \operatorname{Diff}^{-} \mathbf{b i r}_{K} L(\operatorname{Spec} A)$ so that $f: X \times{ }_{C} \operatorname{Spec} A \cdots \rightarrow X \times_{C} \operatorname{Spec} A$ is a $K \otimes_{C} A$-pseudo-automorphism compatible with derivation. By Proposition (1.10), there exist universally $K \otimes_{\mathbf{C}} A$-scheme theoretic dense ope sets $U, V$ of $X_{A}=\operatorname{Spec} K\left[y, y^{-1}\right] \otimes_{\mathbf{C}} A$ such that the pseudo-morphism $f$ induces a $K \otimes_{\mathrm{C}} A$-isomorphism $f: U \rightarrow V$ of schemes compatible with derivation. Let us set

$$
\begin{aligned}
& u=\text { the restriction to } U \text { of } y \in \Gamma\left(X_{A}, \mathfrak{O}\right), \\
& v=\text { the restriction to } V \text { of } y \in \Gamma\left(X_{A}, \mathscr{O}\right), \\
& w=f^{*} v .
\end{aligned}
$$

Since $y$ is invertible on $X$ and hence on $X_{A}$, the functions $u, v$ and hence $w$ are invertible respectively over $U$ or $V$. It follows from the differential equation (2.1.1) that we have

$$
d u / d x=u, \quad d v / d x=v \text { and } \quad d w / d x=w .
$$

The functions $u, w$ are in a differential algebra $\Gamma(U, \mathcal{O})$ and invertible in the algebra. We show that there exists a constant $c$ in the differential algebra $\Gamma(U, \mathbb{O})$ such that $w=c u$. In fact $\left(w u^{-1}\right)^{\prime}=\left(w^{\prime} u-w u^{\prime}\right) / u^{2}=(w u-u w) / u^{2}$ $=0$ by the differential equations (2.1.2). It follows from Proposition (1.11) that any constant of $\Gamma(U, \mathscr{O})$ comes from $\Gamma\left(\operatorname{Spec} A, \mathscr{O}_{\operatorname{Spec~} A}\right)$ through the structure morphism $X_{A} \rightarrow \operatorname{Spec} A$. Since $c$ is invertible, we conclude that $w=c u$ with $c \in$ $A^{*}=$ group of the units of $A$. This shows that the $K \otimes_{\mathbf{C}} A$-isomorphism $f: U \rightarrow$ $V$ coincides with ${ }^{a} \varphi: X_{A} \rightarrow X_{A}$ associated with a $K \otimes_{\mathbf{C}} A$-automorphism $\varphi: K[y$, $\left.y^{-1}\right] \otimes_{\mathbf{C}} A=\left(K \otimes_{\mathbf{C}} A\right)\left[y, y^{-1}\right] \rightarrow K\left[y, y^{-1}\right] \otimes_{\mathbf{C}} A=\left(K \otimes_{\mathbf{C}} A\right)\left[y, y^{-1}\right]$ sending $y$ to $c y$ of the differential algebra. Conversely every element $c \in A^{*}$ defines a $K \otimes_{\mathbf{C}} A$-automorphism $\varphi$ of the differential algebra $K\left[y, y^{-1}\right] \otimes_{\mathbf{C}} A$ sending $y$ to $c y$ and hence $K \otimes_{\mathbf{C}} A$-automorphism ${ }^{a} \varphi: X_{A} \rightarrow X_{A}$ of schemes with derivation. We

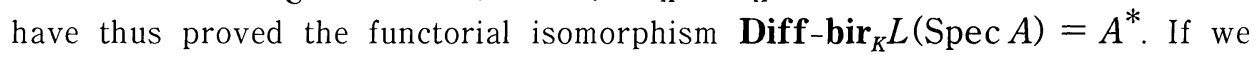
notice that the above argument works not only for affine $\mathbf{C}$-schemes but also for every C-schemes, we have proved a functorial isomorphism $\operatorname{Diff}_{\text {-bir }} L(S)=$ $\Gamma\left(S, \mathfrak{O}_{S}\right)^{*}$ for any $\mathbf{C}$-scheme $S$. Namely the functor $\mathbf{D i f f}$-bir ${ }_{K} L(S)$ is represent- 
able by a group scheme $\mathbf{G}_{m c}$.

We shall recall in $\S 3$, Definition (3.2) the notion of strongly normal extension introduced by Kolchin. The above extension $L / K$ is strongly normal (cf. Example (3.2)). We shall see later that in general if $L / K$ is a strongly normal extension with Galois group $G$ so that $G$ is an algebraic group over $C_{L}=C_{K}$, then the functor $\mathbf{D i f f}-\mathbf{b i r}_{K} L$ is representable by the Galois group $G$ (cf. Theorem (2.22))

Let $G$ be a $C_{K}$-group scheme and $\varphi: G \rightarrow$ Diff-bir ${ }_{K} L$ be a morphism of group functors. Then taking the value at $G \in$ ob $\left(\mathrm{Sch} / C_{K}\right)$, we get a mapping $G(G)=$ $\operatorname{Hom}(G, G) \rightarrow \mathbf{D i f f}-\mathbf{b i r}_{K} L(G) \subset$ Ps.aut $_{G_{K}}\left(X \times G_{C_{K}}\right)$. The image of the identity in $G(G)=\operatorname{Hom}(G, G)$ defines a $G_{K}$-pseudo morphism $X \times_{K} G_{K} \cdot \rightarrow X \times_{K} G_{K}$ of schemes with derivation. By the argument of Demazure [D], p. 514, the group scheme $G_{K}$ with derivation pseudo-operates on $X$. Conversely a pseudo-operation of $G_{K} \times{ }_{K} X \cdots \rightarrow G_{K} \times{ }_{K} X$ which is a $G_{K}$-pseudo-morphism compatible with de-

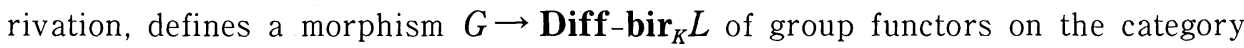
(Sch $/ C_{K}$ ) of $C_{K}$-schemes. We have proved the following

Proposition (2.2). Let $G$ be a group scheme over $C=C_{K}$. Then there is a 1:1-correspondence between the elements of the following two sets.

(i) The set of morphisms $G \rightarrow$ Diff-bir ${ }_{K} L$ of group functors on the dual $(\mathrm{Sch} / C)^{0}$ of the category of $C$-schemes.

(ii) The set of pseudo-operations $G_{K} \times_{K} X \cdots \rightarrow G_{K} \times_{K} X$ that are $G_{K}$-pseudomorphisms compatible with derivation.

Let $L / K$ be a differential field extension satisfying the finiteness condition (F.C) of $\S 1$. We define a functor $\mathscr{F}_{L / K}$ of the category (Fld $/ K^{\natural}$ ) of abstract field extensions of $K^{\natural}$ to the category (Set) of sets by

$$
\begin{aligned}
& \mathscr{F}_{L / K}(A)=\{f \in \operatorname{Diff}-\operatorname{hom}\left(L, A[[t]]\left[t^{-1}\right]\right) \mid \text { the restriction of } f \text { to the sub- } \\
& \text { field } K \text { of } L \text { coincides with the universal Taylor morphism } \\
& i: K \rightarrow K^{\natural}[[t]] \text {. Namely } f \text { makes the diagram } \\
& \qquad \stackrel{f}{\rightarrow} A[[t]]\left[t^{-1}\right] \\
& \uparrow \stackrel{i}{\uparrow} K^{\natural}[[t]]
\end{aligned}
$$

commutative, where the vertical arrows are the natural inclusion morphisms\}. 
for $A \in \operatorname{ab}\left(\mathrm{Fld} / K^{\natural}\right)$.

Here we regard the field $A[[t]]\left[t^{-1}\right]$ of Laurent series as a differential field by the derivation $d / d t$ (cf. $\S 1$ ).

In fact $\mathscr{F}_{L / K}$ is a functor. For if $\varphi: A \rightarrow B$ be a morphism in the category (Fld $/ K^{\natural}$ ) or $\varphi$ is a $K^{\natural}$-morphism of field extensions of $K^{\natural}$, then it induces a morphism $\varphi[[t]]: A[[t]]\left[t^{-1}\right] \rightarrow B[[t]]\left[t^{-1}\right]$ of the fields of Laurent series and hence we get a map $\mathscr{F}_{L / K}(A) \rightarrow \mathscr{F}_{L / K}(B), \varphi \mapsto \varphi[[t]] \circ f$ for every $f: L \rightarrow$ $A[[t]]\left[t^{-1}\right]$ of $\mathscr{F}_{L / K}(A)$ so that $\mathscr{F}_{L / K}:\left(\right.$ Fld $\left./ K^{\natural}\right) \rightarrow($ Set $)$ is a functor.

Remark (2.3.1). For a field extension $A$ of $L^{\natural}$ (so that $A \in \operatorname{ded}\left(\mathrm{Fld} / K^{\natural}\right)$ ), the easiest way to get an element of $\mathscr{F}_{L / K}(A)$ is as follows. By Proposition (1.4) the inclusion $A \supset L^{\natural}$ gives us a Taylor morphism $f: L \rightarrow A[[t]]$ such that the diagram

$$
\begin{array}{ccc}
L^{\natural} & \rightarrow & A[[t]] \\
\uparrow & \uparrow \\
K^{\natural} & \rightarrow & K^{\natural}[[t]]
\end{array}
$$

is commutative, where the vertical arrows are the canonical morphisms. Thus the morphism $f$ composed with the canonical inclusion $A[[t]] \subset A[[t]]\left[t^{-1}\right]$ gives an element of $\mathscr{F}_{L / K}(A)$.

Let $X$ be a differential model of $L / K$. Then $f$ defines a morphism ${ }^{a} f$ : $\operatorname{Spec} A[[t]]\left[t^{-1}\right] \rightarrow \operatorname{Spec} L$ of schemes with derivation and we have the morphism Spec $L \rightarrow X$ of schemes with derivation giving the generic point of $X$. So we have

$\mathscr{F}_{L / K}(A)=\left\{h \in \operatorname{Diff}-\right.$ hom $\left(\operatorname{Spec} A[[t]]\left[t^{-1}\right], X\right) \mid h$ factors through the generic point $\operatorname{Spec} L \rightarrow X$ making the diagram

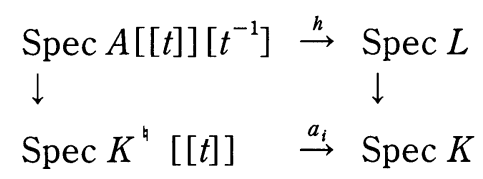

commutative, where the vertical arrows are associated with canonical inclusion morphisms\}.

Since $A[[t]]\left[t^{-1}\right]$ is a field, the last condition is equivalent to requiring that the image of $h: \operatorname{Spec} A[[t]]\left[t^{-1}\right] \rightarrow X$ is the generic point and we have consequently 


$$
\mathscr{F}_{L / K}(A)=\left\{h \in \text { Diff-hom }\left(\operatorname{Spec} A[[t]]\left[t^{-1}\right], X\right) \mid \text { The image of } h:\right.
$$
Spec $A[[t]]\left[t^{-1}\right] \rightarrow X$ is the generic point of $X$ and $h$ makes the diagram

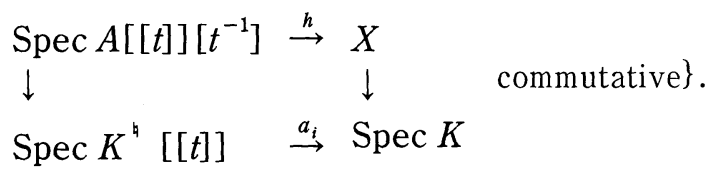

Here the vertical arrows are canonical morphisms induced by the inclusions.

The field $C=C_{K}$ of constants of $K$ is a subfield of $K$ so that $C \subset K^{\natural}$ and the category (Fld $\left./ K^{\natural}\right)$ of $K^{\natural}$-fields is a subcategory of the category (Fld $/ C$ ) of $C$-fields. Since the category $(\mathrm{Fld} / C)$ is a sub-category of the dual category $(\mathrm{Sch} / C)^{0}$, the category $\left(\mathrm{Sch} / K^{\natural}\right)$ is a sub-category of the dual category $(\mathrm{Sch} / C)^{0}$.

Let $G$ be a group sub-functor of $\operatorname{Diff}-\mathbf{b i r}_{K} L:(\mathrm{Sch})^{0} \rightarrow(\mathrm{Grp})$, and $A \in$ ole $\left(\right.$ Fld $\left./ K^{4}\right)$. We can speak of the value $G(\operatorname{Spec} A)$ which we sometimes denote by $G(A)$. Let $g \in G(A)$ so that $g$ defines a Spec $K \otimes_{C_{K}} A$-pseudo automorphism $\varphi_{g}: X_{A} \cdots \rightarrow X_{A}$ of a scheme with derivation. If $h \in \mathscr{F}_{L / K}(A)$, as we noticed above $h: \operatorname{Spec} A[[t]]\left[t^{-1}\right] \rightarrow X$ is a morphism of schemes with derivation and hence we get an $A$-morphism $h_{A}: \operatorname{Spec} A[[t]]\left[t^{-1}\right] \rightarrow X_{A}$ of schemes with derivation. If we can composite $h_{A}$ and $\varphi_{g}$ to get a morphism $\varphi_{g} h_{A}$ : Spec $A[[t]]\left[t^{-1}\right] \rightarrow X_{A}$, we get $p \circ \varphi_{g}{ }^{\circ} h_{A}: \operatorname{Spec} A[[t]]\left[t^{-1}\right] \rightarrow X$ which is a morphism of schemes with derivation, where $p$ denotes the canonical projection $X_{A} \rightarrow X$.

Definition (2.5). (1) Let $G$ be a group sub-functor of the group functor Diff-bir ${ }_{K} L:\left(\mathrm{Sch} / C_{K}\right)^{0} \rightarrow($ Set) satisfying the following conditions: (i) We can composite $\varphi_{g}$ and $h_{A}$; (ii) The image of $p \circ \varphi_{g} \circ h_{A}$ : Spec $A[[t]]\left[t^{-1}\right] \rightarrow X$ is the generic point of $X$ so that $p \circ \varphi_{g} \circ h_{A} \in \mathscr{F}_{L / K}(A)$ and the group functor $G \mid\left(\right.$ Fld $\left./ K^{\natural}\right)$ operates on the functor $\mathscr{F}_{L / K}$.

Then we say that $G \mid\left(\mathrm{Fld} / K^{\natural}\right)$ naturally operates on $\mathscr{F}_{L / K}$ and the morphism $p \circ \varphi_{g} \circ h_{A}$ will be denoted by $g h$. Dually let $f: L \rightarrow A[[t]]\left[t^{-1}\right]$ be the morphism of differential algebras defining $h$. The morphism $L \rightarrow A[[t]]\left[t^{-1}\right]$ of differential algebras corresponding to $p^{\circ} \varphi_{g}{ }^{\circ} h_{A}$ will be denoted by $g f$.

(2) If there exists a group sub-functor of the group functor $\mathbf{D i f f}-\mathbf{b} \mathbf{i r}_{K} L_{K}$ : $\left(\mathrm{Sch} / C_{K}\right) \rightarrow(\mathrm{Grp})$ representable by an algebraic group scheme $G$ such that (i) $G \mid\left(\right.$ Fld $\left./ K^{\natural}\right)$ naturally operates on $\mathscr{F}_{L / K}$ and (ii) $\left(G \mid\left(\right.\right.$ Fld $\left.\left./ K^{\natural}\right), \mathscr{F}_{L / K}\right)$ is a principal homogeneous space, then we say that the extension $L / K$ is quasi- 
automorphic. The group scheme $G$ will be called an automorphy group of the extension $L / K$.

When $G \mid\left(\right.$ Fld $\left./ K^{\natural}\right)$ naturally operates on $\mathscr{F}_{L / K}$, by abuse of language we sometimes say that the group scheme $G$ naturally operates on $\mathscr{F}_{L / K}$.

We notice that in Definition (2.5.1) if the condition is satisfied, then $p^{\circ} \varphi_{g}{ }^{\circ} h_{A}$ makes the diagram

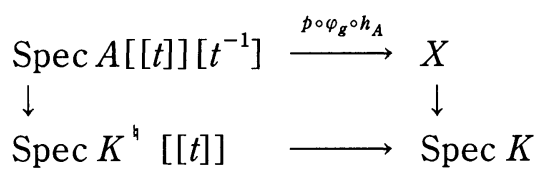

commutative, the vertical arrows being the canonical morphisms.

The uniqueness of the automorphy group is discussed at the end of this section and in $\S 4$ (Theorem (2.22) and Example (4.4)). For a quasi-automorphic extension in general the automorphy group is not uniquely determined.

Let us illustrate the definition by examples.

Example (2.6.1). Let us consider the differential field extension $L / K$ of Example (2.1). We proved there

$$
\operatorname{Diff-} \mathbf{b i r}_{K} L(S)=\Gamma\left(\mathrm{S}, \mathscr{O}_{S}\right)^{*}
$$

for any $C$-scheme $S$. The group functor $\mathbf{D i f f}-\mathbf{b i r}_{K} L$ is representable by the multiplicative group scheme $\mathbf{G}_{m}$ c. Let us take the functor Diff-bir $K_{K} L$ itself as the automorphy group scheme $G$. In particular we have

$$
\operatorname{Diff}_{-\operatorname{bir}_{K}} L(A)=A^{*}
$$

for any field extension $A$ of $K^{\natural}$. Since $K^{\natural}$ is an extension of $C_{K}$, the field $A$ is an extension of $C_{K}$. If we take the model $X=\operatorname{Spec} K\left[y, y^{-1}\right]$, an element $c \in A^{*}$ defines an $A \otimes_{C_{K}} K$-automorphism $\varphi_{c}$ of a differential algebra $A \otimes_{C_{K}} K\left[y, y^{-1}\right]$ sending $y$ to $c y$ and hence an $A \otimes_{C_{K}} K$-automorphism $\varphi_{c}$ of scheme $X \otimes_{C_{K}} A$ with derivation. Let us show that $\mathbf{G}_{m C_{K}}$ operates naturally on $\mathscr{F}_{L / K}$. Let $h:$ Spec $A[[t]]\left[t^{-1}\right] \rightarrow X$ be an element of $\mathscr{F}_{L / K}(A)$. The corresponding morphism $K[y$, $\left.y^{-1}\right] \rightarrow A[[t]]\left[t^{-1}\right]$ of differential algebras is denoted by $f$. Since $\varphi_{C}$ is an automorphism of $X_{A}$ over $K \otimes_{C_{K}} A, \varphi_{c}{ }^{\circ} h_{A}: \operatorname{Spec} A[[t]]\left[t^{-1}\right] \rightarrow X_{A}$ and hence $p^{\circ} \varphi_{c}{ }^{\circ} h_{A}$ : Spec $A[[t]]\left[t^{-1}\right] \rightarrow X$ are defined. The morphism $p^{\circ} \varphi_{C}{ }^{\circ} h_{A}: \operatorname{Spec} A[[t]]\left[t^{-1}\right] \rightarrow X$ is associated with a morphism $k: K\left[y, y^{-1}\right] \rightarrow A[[t]]\left[t^{-1}\right]$ sending $y$ to $c f(y)$ and extending the canonical morphism $i: K \rightarrow K^{\natural}[[t]] \subset A[[t]]\left[t^{-1}\right]$. Thus it suffices to check that the morphism $k$ factors through the generic point. Or equivalently $k$ 
can be extended to the quotient field $L=K(y)$ of $K\left[y, y^{-1}\right]$. In a differential algebra $\mathbf{Q}(f(L) . A), f(L)$ and $C_{\mathbf{Q}^{(f(L) . A}}=A$ are linearly disjoint over $f\left(C_{L}\right)=$ $f(C)=C\left(\subset K^{\natural}[[t]] \subset A[[t]]\left[t^{-1}\right]\right)$ by Lemma (1.1) so that $\mathbf{Q}(f(L) . A)=$ $\mathbf{Q}\left(f(L) \otimes_{C_{K}} A\right)$. For a similar reason $\mathbf{Q}(f(K) . A) \simeq \mathbf{Q}\left(f(K) \otimes_{C} A\right)$. Since $0 \neq c$ $\in A$, there exists a $\mathbf{Q}\left(i(K) \otimes_{C} A\right)$-automorphism $\xi_{C}: \mathbf{Q}\left(f(L) \otimes_{C} A\right) \rightarrow \mathbf{Q}(f(L)$ $\left.\otimes_{C} A\right)$ of the differential field sending $f(y)$ to $c f(y)$ and hence we have an $\mathbf{Q}(f(K) . A)$-automorphism $\phi_{c}$ of the differential field $\mathbf{Q}(f(L) . A)$ sending $f(y)$ to $c f(y)$ by the above isomorphisms. Consequently $\phi_{c} \circ f: L \rightarrow \mathbf{Q}(f(L) A) \rightarrow$ $\mathbf{Q}(f(L) A) \subset A[[t]]\left[t^{-1}\right]$ is the desired extension of $k$.

Let now $h_{\imath}: \operatorname{Spec} A[[t]]\left[t^{-1}\right] \rightarrow X(i=0,1)$ be elements of $\mathscr{F}_{L / K}(A)$. The morphisms $h_{0}, h_{1}$ arise from morphisms $f_{i}: K\left[y, y^{-1}\right] \rightarrow A[[t]]\left[t^{-1}\right](i=0,1)$ of differential algebras factorizing through the morphism $K\left[y, y^{-1}\right] \rightarrow L$ which gives the generic point of $X=\operatorname{Spec} K\left[y, y^{-1}\right]: h_{i}={ }^{a} f_{i}$ for $i=0,1$. It follows from the differential equation (2.1.1) that $k_{i}(y)^{\prime}=\frac{d k_{i}(y)}{d t}=k_{\imath}(y)$ so that $\frac{d k_{0}(y)^{-1} k_{1}(y)}{d t}=\frac{k_{1}(y)^{\prime} k_{0}(y)-k_{1}(y) k_{0}(y)^{\prime}}{k_{0}(y)^{2}}=\frac{k_{1}(y) k_{0}(y)-k_{1}(y) k_{0}(y)}{k_{0}(y)^{2}}=0$. So $k_{0}(y)^{-1} k_{1}(y)$ is a constant in $A[[t]]\left[t^{-1}\right]$ : There exists a non-zero element $c \in A^{*}$ such that $k_{1}(y)=c k_{0}(y)$. This shows $p \circ \varphi_{c}{ }^{\circ}{ }^{a} f_{0}={ }^{a} f_{1}$ and $\mathscr{F}_{L / K}$ is a principal homogeneous space of $\mathbf{G}_{m c_{K}}$. So $L / K$ is a quasi-automorphic extension.

(2.6.2) The second example is Galois extension of abstract field. Let $L$ be a (finite) Galois extension of an abstract field $K$. They are differential fields with trivial derivation. Since $L / K$ is finite, Spec $L$ is the unique model of $L / K$. Since $C_{K}=K,\left(\mathrm{Sch} / C_{K}\right)=(\mathrm{Sch} / K)$ so that the functors $\operatorname{Diff}_{-} \mathbf{b i r}_{K} L, \mathbf{B i r}_{K} L, \mathbf{A u t}_{K} L$ : $(\mathrm{Sch} / K)^{0} \rightarrow(\mathrm{Grp})$ coincide. Here for a $K$-scheme $X$ the following notation is employed: The functors $\mathbf{A u t}_{K} X$ and $\mathbf{B i r}_{K} X$ are respectively the functor of automorphisms of the $K$-scheme $X$ and the functor of pseudo-automorphisms of the scheme $X$

$$
\operatorname{Aut}_{K} X(S)=\operatorname{Aut}_{S} X \times_{K} S \text { and } \operatorname{Bir}_{K} X(S)=\text { Ps.aut }_{S} X \times_{K} S
$$

for any $K$-scheme $S$. The Galois group $G$ of the extension $L / K$ is a finite group so that we can regard it as a finite group scheme over $K$. The finite group scheme $G$ operates on the scheme $\operatorname{Spec} L$. So $G$ is a representable subgroup functor of Diff-bir ${ }_{K} L$. As is well-known $(G, \operatorname{Spec} L)$ is a principal homogeneous space, when we consider $G$ and $\operatorname{Spec} L$ as functors on the category $(\operatorname{Sch} / K)^{0}$. For any field extension $A$ of $C_{K}=K$, $\operatorname{Diff-hom}\left(L, A[[t]]\left[t^{-1}\right]\right)=\operatorname{Hom}(L, A)$ so that $\mathscr{F}_{L / K}(A)=\operatorname{Hom}_{K}(L, A)$ is a principal homogeneous space of $G(A)$ which is no- 
thing but the finite group $G$. We have proved that every Galois extension of an abstract field with Galois group $G$ is quasi-automorphic with automorphy group $G$.

We can show the converse of Example (2.6.2).

THEOREM (2.7). Let $L / K$ be an abstract field extension. Then the following conditions are equivalent.

(1) The extension $L / K$ is quasi-automorphic.

(2) There exists a finite group scheme $G$ over $K$ which operates on a model $X$ of $L / K$ such that $(G, X)$ is a principal hemogeneous space, $X$ and $G$ being regarded as a functor on the category $(\mathrm{Sch} / K)^{0}$ of $K$-schemes.

(3) There exists a finite group scheme $G$ over $K$ which operates on $\operatorname{Spec} L$ over $K$ such that $(G, \operatorname{Spec} L)$ is a principal hemogeneous space.

Proof. If the condition (2) is satisfied, then $X$ is finite over $K$ and hence $L$ is finite over $K$ and $X=\operatorname{Spec} L$ so that the condition (3) is satisfied.

If the condition (3) holds, then $\operatorname{Spec} L$ is finite over $K$, hence $\operatorname{Spec} L$ is a model of $K$ and condition (2) is satisfied with $X=\operatorname{Spec} L$. The argument of Example (2.6.2) allows us to prove that the condition (3) implies the condition (1) since $L$ is finite over $K$ if condition (3) is assumed.

Let us now assume the condition (1). We first show that $L$ is finite over $K$. Let us examine the conditions (2.5.1), (2.5.2) for an abstract case. First of all $C_{K}=K$ and the canonical morphism $i: K \rightarrow K^{\natural}[[t]]$ is the identification of $K$ with the ring of constant series in $K^{\natural}[[t]]$. For a field $A \in$ ob (Fld $/ K$ ), Diff-hom $(L, A[[t]])=\operatorname{Hom}(L, A)$ and the diagram (2.3) reduces to

$$
\begin{aligned}
& L \rightarrow A \rightarrow A[[t]]\left[t^{-1}\right] \\
& \uparrow \uparrow \uparrow \\
& K \rightarrow K \rightarrow K[[t]]
\end{aligned}
$$

so that $\mathscr{F}_{L / K}(A)=\operatorname{Hom}_{K}(L, A)$. It follows from Proposition (1.17) that we can find a model $X$ of $L / K$ such that the group scheme $G$ of automorphy operates on $X$ over $K$. We need

Lemma (2.8). The operation $(G, X)$ is generically transitive, i.e. the morphism $G$ $\times_{C} X \rightarrow X \times{ }_{K} X,(g, x) \mapsto(g x, x)$ is dominant.

Proof of Lemma. It is sufficient to show that the base changed operation $\left(G_{\bar{K}}\right.$, $\left.X_{\bar{K}}\right)$ is generically transitive for an algebraic closure $\bar{K}$ of $K$. The total quotient ring $\mathbf{Q}\left(L \otimes_{K} \bar{K}\right)$ is a direct product of fields $M_{i}: \mathbf{Q}\left(L \otimes_{K} \bar{K}\right)=\Pi_{i=1}^{n} M_{i}$. Let $X_{i}$ 
be a model of $M_{i} / \bar{K}$. Then $X_{\bar{K}}=X \otimes_{K} \bar{K}$ is $\bar{K}$-birationally equivalent to disjoint union of the $X_{i}$ : There exists $\bar{K}$-isomorphism between Zariski open sets of $X_{\bar{K}}$ and $\amalg_{i=1}^{n} X_{i}$. Let $\Omega$ be a field extension of $\bar{K}$ and $p, q: \amalg_{i=1}^{n} X_{i}$ be generic points of $\amalg_{i=1}^{n} X_{i}$, i.e. $p$ and $q$ are respectively a generic point of $X_{i}$ and $X_{j}$ for some indices $1 \leq i, j \leq n$. We have possibly $i=j$. The morphism $p$ is defined by a $\bar{K}$ -morphism $M_{i} \rightarrow \Omega$. Composing with the canonical morphisms we get a $K$ morphism $p^{*}: L \rightarrow \mathbf{Q}\left(L \otimes_{K} \bar{K}\right) \rightarrow M_{i} \rightarrow \Omega$. Similarly $q$ gives us a $\bar{K}$-morphism $q^{*}: L \rightarrow \mathbf{Q}\left(L \otimes_{K} \bar{K}\right) \rightarrow M_{j} \rightarrow \Omega$. Conversely the morphisms $p^{*}$ and $q^{*}$ determine the points $p$ and $q$. Since $p^{*}$ and $q^{*}$ are in $\mathscr{F}_{L / K}(\Omega)$, it follows from condition (1) that there exists a point $g \in G(\Omega)$ such that $g p^{*}=q^{*}$ or $g p=q . \quad$ q.e.d.

It follows from Lemma (2.8) that we may assume that $(G, X)$ is a homogeneous space.

Lemma (2.9). We can find a model $X$ of $L / K$ such that $(G, X)$ is a principal homogeneous space.

Proof of Lemma. In fact in the proof of Lemma (2.8), the choice of $g \in$ $G(\Omega)$ is unique.

Now we come back to the proof of the theorem. We take a model $X$ such that $(G, X)$ is a principal homogeneous space. We can find a $\bar{K}$-valued point of the $K$-algebraic scheme $X$ by the Hilbert Nullstellensatz. So there exist a finite extension $N$ of $K$ and a $N$-valued point $q: \operatorname{Spec} N \rightarrow X$. This gives an $\bar{L}$-valued point $\bar{q}$ : Spec $\bar{L} \rightarrow X$ since $N \subset \bar{K}$ is contained in an algebraic closure $\bar{L}$ of $L$. On the other hand the inclusion $j: L \rightarrow \bar{L}$ or the associated morphism $p: \operatorname{Spec} \bar{L} \rightarrow \operatorname{Spec}$ $L \rightarrow X$ lies in $\mathscr{F}_{L / K}(\bar{L})$. Denoting the $\bar{L}$-rational points of $X_{\bar{L}}$ corresponding to $\bar{q}$ : Spec $\bar{L} \rightarrow X$ and $p:$ Spec $\bar{L} \rightarrow X$ by the same letter $\bar{q}$ and $p$, there exists the unique $g \in G(\bar{L})$ such that $g p=\bar{q}$ since $(G, X)$ is a principal homogeneous space. Thus $\bar{q} \in \mathscr{F}_{L / K}(\bar{L})$ by our assumtion of being automorphic so that $\bar{q}$ : Spec $\bar{L} \rightarrow X$ and consequently also $q$ : Spec $N \rightarrow X$ factors through the generic point. Thus the extension $L / K$ is contained in the finite algebraic extension $N$ so That $L / K$ is finite, $X=\operatorname{Spec} L$ and $G$ is a finite group scheme.

Example (2.10). Let $K$ be an abstract field or a field with trivial derivation such that $u^{2}+u+1$ is irreducible in the polynomial ring $K[u]$, i.e. any primitive cube root of 1 is not in $K$. Let $a$ be an element of $K$ such that a polynomial $u^{3}-a$ is irreducible in $K[u]$. Let $L=K[u] /\left(u^{3}-a\right)$ and $\bar{u}$ the residue class of $u$ so 
that $L=K[\bar{u}]$. The field $L$ is an extension of $K$ of degree 3. Let $G$ be the group scheme of the cube root of 1 or let $G$ be the kernel of the morphism $\mathbf{G}_{m K} \rightarrow \mathbf{G}_{m K}$, $v \mapsto v^{3}$ for $v \in \mathbf{G}_{m K}$. As a scheme $G=\operatorname{Spec} K[z]\left(z^{3}-1\right)=\operatorname{Spec} K[\bar{z}], \bar{z}$ being the residue class of $z$ and the multiplication $G \times G \rightarrow G$ is given by $K[\bar{z}] \rightarrow$ $K[\bar{z}] \otimes K[\bar{z}]$ sending $\bar{z}$ to $\bar{z} \otimes \bar{z}$. The group scheme $G$ operates on a scheme Spec $L$ over $K$ by $K$-morphism $K[\bar{u}] \rightarrow K[\bar{z}] \bigotimes_{K} K[\bar{u}]$ sending $\bar{u}$ to $\bar{z} \otimes \bar{u}$. (G, Spec $L)$ is a principal homogeneous space and hence $L / K$ is quasi-automorphic. But $L / K$ is not Galois since any primitive cube root of 1 is not contained in $K$.

Remark (2.11). Abstract field extensions which are quasi-automorphic were studied systematically in [G]. Disadvantages of such a Galois theory are that the automorphy group is not uniquely determined and we do not have Galois correspondence. A detailed discussion will be done in $\S 4$.

Let $L / K$ be a quasi-automorphic extension with group $G$ of automorphy. Let $X$ be a model with derivation of the extension $L / K$ so that the algebraic group $G_{K}$ pseudo-operates on $X$ : We have an $X$-pseudo-morphism $\left(\mu, p_{2}\right): G_{C_{K}} \times{ }_{C_{K}} X=$ $G_{K} \times_{K} \times \cdots \rightarrow X \times_{K} X$ sending $(g, x)$ to $(g x, x)$ of schemes with derivation. Here two schemes are regarded as $X$-schemes via the second projections.

Lemma (2.12). If $L / K$ is quasi-automorphic, then $\left(G_{K}, X\right)^{\natural}$ is pseudotransitive. More precisely the pseudo-morphism $\left(\mu, p_{2}\right): G \times{ }_{C} X \cdots \rightarrow X \times{ }_{K} X$ is dominant.

Proof. It is sufficient to show that $\left(G_{\bar{K}^{\natural}}, X_{\bar{K}}^{\natural}\right)$ is generically transitive for an algebraic closure $\bar{K}$ of $K$. The total quotient ring $\mathbf{Q}\left(L \bigotimes_{K} \bar{K}\right)$ is a direct product of differential fields $M_{\imath}: \mathbf{Q}\left(L \otimes_{K} \bar{L}\right)=\Pi_{i=1}^{n} M_{i}$. Let $X_{i}$ be a differential model of $M_{i} / \bar{K}$. Then $X_{\bar{K}}=X \otimes_{K} \bar{K}$ is differentialy $\bar{K}$-birationally equivalent to the disjoint union $\amalg_{i=1}^{n} X_{i}$ of the schemes $X_{i}$ : There exists a $\bar{K}$-isomorphism of schemes with derivation between dense Zariski open sets of $X_{\bar{K}}$ and $\amalg_{i=1}^{n} X_{\imath}$. Let $\Omega$ be a field extension of $\bar{K}^{\natural}$ and $p, q: \operatorname{Spec} \Omega \rightarrow \amalg_{i=1}^{n} X_{i}^{\natural}$ be generic points of $\amalg_{i=1}^{n} X_{i}^{\natural}$ i.e. the images of the morphisms $p$ and $q$ are respectively the generic points of $X_{i}$ and $X_{j}$ for some indices $1 \leq i, j \leq n$. We have possibly $i=j$. The morphism $p$ is defined by $\bar{K}^{\natural}$-morphism $M_{i}^{\natural} \rightarrow \Omega$. Composing with the canonical morphisms we get a $K^{\natural}$-morphism $p^{*}: L^{\natural} \rightarrow \mathbf{Q}\left(L \otimes_{K} \bar{K}\right)^{\natural} \rightarrow M_{i}^{\natural} \rightarrow \Omega$ and consequently a morphism $p^{*}[[t]]: L^{\natural}[[t]] \rightarrow \Omega[[t]] \subset \Omega[[t]]\left[t^{-1}\right]$ of differential algebras. Composing now $p^{*}[[t]]$ with the universal Taylor morphism $i: L \rightarrow L^{\natural}[[t]]$ we get a morphism $\tilde{p}: L \rightarrow \Omega[[t]]\left[t^{-1}\right]$ so that $\tilde{p} \in \mathscr{F}_{L / K}(\Omega)$. Similarly for $q$, we get a 
morphism $q^{*}: L^{\natural} \rightarrow \Omega$ of fields and a morphism $\tilde{q}: L \rightarrow \Omega[[t]]\left[t^{-1}\right]$ of differential fields such that $\tilde{q} \in \mathscr{F}_{L / K}(\Omega)$. So there exists $g \in G(\Omega)$ such that $g \tilde{p}=\tilde{q}$. Since $\tilde{p}$ and $\tilde{q}$ factor through the subring $\Omega[[t]] \subset \Omega[[t]]\left[t^{-1}\right], g \tilde{p}=\tilde{q}$ implies by a specialization $t=0 \mathrm{~g} p=q$.

THEOREM (2.13). Let $L / K$ be a differential field extension satisfying the finiteness condition (F.C) of $\S 1$. Then the following conditions (1) and (2) are equivalent.

(1) The extension $L / K$ is quasi-automorphic with automorphy group $G$.

(2) The extension $C_{L} / C_{K}$ is finite and we can find a model with derivation $X$ of $L / K$ such that an algebraic $C_{K}$-group scheme $G$ operates on $X$ differentially, i.e. the morphism $\mu: G \times{ }_{C_{K}} X=G_{K} \times_{K} X \rightarrow X,(g, x) \mapsto g x$ of the operation is a morphism of $K$-schemes with derivation and such that $\left(G_{K^{4}}, X^{\natural}\right)$ is a principal homogeneous space.

Remark. (2.13.11). Before we start proof, we notice that the latter condition in (2) is equivalent to the morphism

$$
\left(\mu, p_{2}\right): G_{K} \times_{K} X \rightarrow X \times_{K} X
$$

is an isomorphism of schemes with derivation (cf. $\S 1$ ).

Proof. If the condition (2) is satisfied, then the morphism $\left(\mu, p_{2}\right): G \times_{C_{K}} X \rightarrow$ $X \times_{K} X$ is a $X$-isomorphism of schemes with derivation, where the both schemes are considered as $X$-schemes via second projections. Let us prove that the group scheme $G$ naturally operates on the functor $\mathscr{F}_{L / K}$ (cf. Definition (2.3)). Let $A \in$ (Fld $\left./ K^{\natural}\right), g \in G(A)$ and let $p: \operatorname{Spec} A[[t]]\left[t^{-1}\right] \rightarrow X$ be a point of $X$ giving an element of $\mathscr{F}_{L / K}(A)$. To simplify the notation, we denote the field $C_{K}$ by $C$. We denote the image of $g: \operatorname{Spec} A \rightarrow G$ by the same letter $g$. The point $g$ induces a $C(g) \otimes_{C} K$-automorphism $\varphi_{g}: C(g) \otimes_{C} X \rightarrow C(g) \otimes_{C} X$ of $C(g) \otimes_{C} K$-scheme $C(g) \otimes_{C} X$ with derivation. Here $C(g)$ denotes the residue field $\mathscr{O}_{G g} / m_{g}$. We have to show that $p_{2}{ }^{\circ} \varphi_{g}{ }^{\circ} p: \operatorname{Spec} A[[t]]\left[t^{-1}\right] \rightarrow C(g) \otimes_{C} X \rightarrow C(g) \otimes_{C} X \rightarrow X$ is in $\mathscr{F}_{L / K}(A)$. To this end it is sufficient to check that the image of $p_{2}{ }^{\circ} \varphi_{g}{ }^{\circ} p$ is the generic point of $X$. We notice here that since the field $C$ is algebraically closed in $K$, the extension $K / C$ is regular so that $C(g) \otimes_{C} K$ is a domain. If we regard $C(g)$ as a subfield of $A[[t]]\left[t^{-1}\right]$ by the morphism $g:$ Spec $A \rightarrow G$ and by the natural inclusion $A \subset A[[t]]\left[t^{-1}\right]$, then $C(g) \otimes_{C} K \simeq C(g) . p^{*} K$ by Lemma (1.1). Since the morphism $\varphi_{g}$ is a $C(g) \otimes_{C} K$-automorphism, if we can show that the image of $p$ is the generic point of an irreducible component of the generic fibre of the $C(g) \otimes_{C} K$-scheme $C(g) \otimes_{C} X$, then the image $\varphi_{g} \circ p:$ Spec $A[[t]]\left[t^{-1}\right] \rightarrow C(g)$ 
$\otimes_{C} X$ is the generic point of an irreducible component of the generic fibre of the $C(g) \otimes_{C} K$-scheme $C(g) \otimes_{C} X$ and hence $p_{2}{ }^{\circ} \varphi \circ p$ is the generic point of $X$. So the question is reduced to proving that the image of $p$ is the generic point of an irreducible component of the generic fibre of the $C(g) \otimes_{C} K$-scheme $C[g] \otimes_{C} X$. The point $g: \operatorname{Spec} A \rightarrow G$ defines a point $g^{\prime}=g{ }^{\circ}{ }^{a} i: \operatorname{Spec} A[[t]]\left[t^{-1}\right] \rightarrow G$, where $i$ denotes the natural inclusion map $i: A \subset A[[t]]\left[t^{-1}\right]$. So we get a point $g^{\prime}:$ Spec $A[[t]]\left[t^{-1}\right] \rightarrow G$ and hence a point $p^{\prime}=\left(g^{\prime}, p\right):$ Spec $A[[t]]\left[t^{-1}\right] \rightarrow G \times{ }_{c} X$. The morphism $p^{\prime}$ factors through $C(g) \otimes_{C} \operatorname{Spec} \mathscr{O}_{X p} \rightarrow G \times_{C} X$, where $p$ is the generic point of $X$ that is the image of the morphism $p: \operatorname{Spec} A[[t]]\left[t^{-1}\right] \rightarrow X$ so that $\mathscr{O}_{X p} \simeq L$. Namely there exists a morphism $p^{\prime \prime}: \operatorname{Spec} A[[t]]\left[t^{-1}\right] \rightarrow C(g) \bigotimes_{C}$ $\operatorname{Spec} \mathscr{O}_{X p}$ such that the diagram

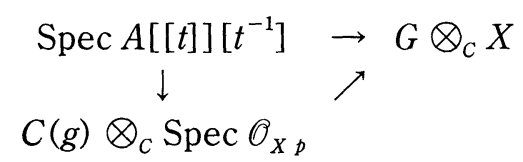

is commutative, where the morphism $C(g) \bigotimes_{C} X \rightarrow G \bigotimes_{C} K$ is the canonical morphism. Since the irreducible components of the generic fibre of the scheme $C(g) \otimes_{C} X$ over $C(g) \otimes_{C} K$ are of dimension tr.d. $[L ; K]$, we have to show that $\operatorname{tr}$. d. $\left[p^{\prime \prime *}\left(C(g) \otimes_{C} \mathscr{O}_{X p}\right) ; C(g) \bigotimes_{C} K\right]=\operatorname{tr}$. d. $[L ; K]$. In fact $p^{\prime \prime *}\left(C(g) \bigotimes_{C} \mathscr{O}_{X p}\right)$ $=g^{\prime *}(C(g)) \cdot p^{*}\left(\mathscr{O}_{X p}\right) \subset g^{\prime *}(C(g)) p^{*}(L) \subset A[[t]]\left[t^{-1}\right]$. It follows from Lemma (1.1) that $g^{\prime *}\left(C(g) \cdot p^{*}(L) \simeq g^{\prime *}(C(g)) \bigotimes_{C^{\prime}} p^{*}(L)=C(g) \bigotimes_{C^{\prime}} L\right.$, where $C^{\prime}=$ $C(g) \cap p^{*}(L)$. So tr.d. $\left[p^{\prime \prime *}\left(C(g) \bigotimes_{C} \mathscr{O}_{X p}\right) ; C(g) \bigotimes_{C} K\right]=\operatorname{tr}$. d. $\left[C(g) \otimes_{C^{\prime}} L\right.$; $\left.C(g) \otimes_{C} K\right]$. Since $K C^{\prime} \simeq \mathbf{Q}\left(K \otimes_{C} C^{\prime}\right)$ by Lemma (1.1), $\mathbf{Q}\left(C(g) \otimes_{C^{\prime}} K C^{\prime}\right) \simeq$ $\mathbf{Q}\left(C(g) \otimes_{C} K\right)$. Hence tr.d. $\left(C(g) \bigotimes_{C^{\prime}} L: C(g) \bigotimes_{C} K\right]=\operatorname{tr}$. d. $\left[C(g) \bigotimes_{C^{\prime}} L ; C(g)\right.$ $\left.\bigotimes_{C^{\prime}} K C^{\prime}\right]=\operatorname{tr}$. d. $\left[\left(C(g) \bigotimes_{C^{\prime}} K C^{\prime}\right) \bigotimes_{K C^{\prime}} L ; C(g) \bigotimes_{C^{\prime}} K C^{\prime}\right]=\operatorname{tr}$. d. $\left[L ; K C^{\prime}\right]$. Since the extension field $C^{\prime}$ is an intermediate field of the finite field extension $C_{L} / C=C_{K}$, the extension $K C^{\prime} / K^{\prime}$ is finite. Consequently tr.d. $\left[L ; K C^{\prime}\right]=\operatorname{tr}$. d. $[L ; K]$ so that $\operatorname{tr} . d .\left[p^{\prime \prime *}\left(C(g) \otimes_{C} \mathscr{O}_{X p}\right) ; C(g) \bigotimes_{C} K\right]=\operatorname{tr}$. d. $[L ; K]$. This is what we had to prove. So the group scheme $G$ operates naturally on the functor $\mathscr{F}_{L / K}$.

Let now $A$ be a field extension of $K^{\natural}$ and $p, g: \operatorname{Spec} A[[t]]\left[t^{-1}\right] \rightarrow X$ be elements of $\mathscr{F}_{L / K}(A)$ so that $p, q$ are morphisms of schemes with derivation making the diagram (2.4) commutative. So $(p, q): \operatorname{Spec} A[[t]]\left[t^{-1}\right] \rightarrow X \times{ }_{K} X$ defines a morphism of schemes with derivation. Thus by the isomorphism (2.13.2), we get a morphism $r: \operatorname{Spec} A[[t]]\left[t^{-1}\right] \rightarrow G \times{ }_{C_{K}} X$ of schemes with derivation. Since Spec $A[[t]]\left[t^{-1}\right] \stackrel{r}{\rightarrow} G \times_{C_{K}} X \stackrel{p_{2}}{\rightarrow} X$ is nothing but the morphism $q, r$ is determined by a composite morphism $s: \operatorname{Spec} A[[t]]\left[t^{-1}\right] \stackrel{r}{\rightarrow} G \times_{C_{K}} X \stackrel{p^{1}}{\rightarrow} G$ of schemes with derivation and we have $s q=p$ by the isomorphism (2.13.2). Since $G$ is a scheme with 
trivial derivation, the morphism $s$ factors through the subring $A \subset A[[t]]\left[t^{-1}\right]$ of constants of $A[[t]]\left[t^{-1}\right]$ so that there exists a point $u: \operatorname{Spec} A \rightarrow G$ such that $r=$ $u \circ \phi$, where $\phi: \operatorname{Spec} A[[t]]\left[t^{-1}\right] \rightarrow \operatorname{Spec} A$ is the canonical morphism. In other words $u q=p$ and the $u \in G(A)$ with $u p=q$ is unique by the isomorphism (2.13.2). Thus $\mathscr{F}_{L / K}(A)$ is a principal homogeneous space of $G(A)$. So the condition (2) implies the condition (1).

Let us now assume that the $L$ is a quasi-automorphic extension of $K$. Then it follows from Propositions (1.15) and (2.2) that we can find a model $X$ of $L^{\natural} / K^{\natural}$ such that $G$ operates on $X$. Since $L$ has the derivation $\delta$ and the operation of $G$ is compatible with the derivation, we have a derivation $\delta: \mathscr{O}_{X} \rightarrow K(X)=L$, where $K(X)$ is the constant sheaf of rational functions on the scheme $X$ such that the operation of $G$ is compatible with $\delta$. So there exists a non-empty $G$-invariant open set $U$ of $X$ such that $\delta: \mathscr{O}_{X} \rightarrow K(X)$ induces a derivation $\mathscr{O}_{U} \rightarrow \mathscr{O}_{U}$. Let us denote $U$ by $X$ again. The algebraic scheme $X$ has the following properties: The algebraic scheme $X$ is a scheme with derivation and is a model of $L / K$. The algebraic group scheme $G_{K}$ operates on $X$ such that the morphism $\mu: G \times_{C} X=G_{K} \times X \rightarrow X$ is a $K$-morphism of schemes with derivation. So the morphism (Id, $\mu$ ) :G $\times_{C} X \rightarrow G$ $\times_{C} K$ is a $G \otimes_{C} K$-automorphism of the scheme $G \times_{C} X$ with derivation. By Lemma (2.12) we may assume that $\left(G_{K^{4}}, X\right)$ is a homogeneous space. In the argument of the proof of Lemma (2.12), the element $g \in G(\Omega)$ such that $g p=q$ is unique so that $\left(G_{K}^{\natural}, X\right)$ is a principal homogeneous space. So the second condition of (2) is satisfied. It remains to show that the extension $C_{L} / C_{K}$ is finite. Let us state it in a form of Lemma.

Lemma (2.14). If $L / K$ is a quasi-automorphic extension, then the field $C_{L}$ of constants of $L$ is a finite extension of $C_{K}$.

Proof. Let $X$ be the differential model of $L / K$ such that the automorphy group $G$ operates on $X$ so that $\left(G_{K}, X\right)^{\natural}$ is a principal homogeneous space. It follows from Lemma (1.2) that the extension $C_{L}$ is finitely generated over $C_{K}$. Let $Y$ be a model of $C_{L} / C_{K}$. Since $C_{L}$ and $K$ are linearly disjoint over $C_{K}, Y_{K}$ is a model of $K C_{L} / K$. So there exists a dominant $K$-rational map $f: X \cdots \rightarrow Y_{K}$ of schemes with derivation. Let $U$ be a Zariski open set of $X$ over which the rational map $f$ is regular so that $f(U) \subset Y_{K}$ contains a dense Zariski open set $W$. Since the field $C_{K}$ of constants of $K$ is algebraically closed in $K, K$ is a regular extension of $C_{K}$ so that $K \otimes_{C_{K}} \bar{C}_{K}$ is a domain for an algebraic closure $\bar{C}_{K}$ of $C_{K}$ and we can consider the quotient field $\mathbf{Q}\left(K \otimes_{C_{K}} \bar{C}_{K}\right)$. 
Sublemma (2.15). There exist a $\bar{C}_{K^{-}}$valued point $q:$ Spec $\bar{C}_{K} \rightarrow Y$ such that the induced $\mathbf{Q}\left(K \otimes_{C_{K}} \bar{C}_{K}\right)$-valued point $q_{K}: \operatorname{Spec} \mathbf{Q}\left(K \otimes_{C_{K}} \bar{C}_{K}\right) \rightarrow \operatorname{Spec} K \otimes_{C_{K}} \bar{C}_{K} \rightarrow Y_{K}$ factors through $W$ or the image $q_{K}\left(\operatorname{Spec} \mathbf{Q}\left(K \otimes_{C_{K}} \bar{C}_{K}\right)\right) \in W$.

Proof of Sublemma. The complement $Z=Y_{K}-W$ is a closed set of $Y_{K}$. Since $X$ and $Y$ are algebraic schemes, we can find a subring $R$ of $K$ of finite type over $C_{K}$ such that $Z \subset Y_{K}$ is defined over $R$. There exist a closed subscheme $Z_{0} \subset Y_{R}$ of $X_{0}$ such that $Z_{0} \otimes_{R} K \subset Y_{K}$ coincides with the closed subscheme $Z$ of $Y_{K}$. To simplify the notation we set $C_{K}=C, \bar{C}_{K}=\bar{C}$ and $\bar{R}=R \otimes_{C} \bar{C}$. The schemes $Y_{\bar{C}}$, Spec $\bar{R}$, and $Y_{\bar{R}}$ are $\bar{C}$-algebraic and hence $Z_{0 \bar{R}}$ is a closed $\bar{C}_{\text {-algebraic subscheme }}$

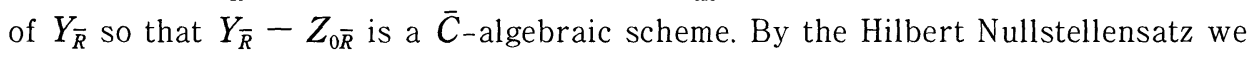
can find a $\bar{C}$-valued point $q^{\prime}: \operatorname{Spec} \bar{C} \rightarrow Y_{\bar{R}}-Z_{0 \bar{R}}$. Thus we get a $\bar{C}$-valued point of $Y$ by composing $q^{\prime}$ with $Y_{\bar{R}}-Z_{0 \bar{R}} \subset Y_{\bar{R}} \rightarrow Y$ : We denote this point by $q$ : Spec $\bar{C} \rightarrow Y$. Then $q$ satisfies our requirement. In fact since $K / C$ is a regular extension, $\bar{R}$ is a domain. $q: \operatorname{Spec} \bar{C} \rightarrow Y$ defines a section $q_{\bar{R}}:$ Spec $\bar{R} \rightarrow Y_{\bar{R}}$. Since the image $q^{\prime}(\operatorname{Spec} \bar{C})$ is in $Y_{\bar{R}}-Z_{0 \bar{R}}$, i.e. $q^{\prime}(\operatorname{Spec} \bar{C})$ is not in $Z_{0 \bar{R}}$ and $q^{\prime}(\operatorname{Spec}$ $\bar{C}) \subset q_{\bar{R}}(\operatorname{Spec} \bar{R})$, the image $q_{\bar{R}}(\operatorname{Spec} \bar{R})$ is not contained in $Z_{0 \bar{Z}}$ so that the image of the generic point of $\operatorname{Spec} \bar{R}$ is not in $Z_{0 \bar{R}}$. Namely the $\mathbf{Q}(\bar{R})$-valued point $q_{\mathbf{Q}^{(\bar{R})}}$ : $\operatorname{Spec} \mathbf{Q}(\bar{R}) \rightarrow Y_{\bar{R}}$ induced by $q$ factors through $Y_{\bar{R}}-Z_{0 \bar{R}}$. Hence the $\mathbf{Q}\left(K \otimes_{C}\right.$ $\bar{C})$-valued point $q_{\left.\mathbf{Q}^{(K} \otimes_{C} \bar{C}\right)}:$ Spec $\left.\mathbf{Q}\left(K \otimes_{C} \bar{C}\right) \rightarrow Y_{\mathbf{Q}^{(K}} \otimes_{C} \bar{C}\right)$ factors through $\left(Y_{\bar{R}}-\right.$ $\left.Z_{0}\right) \otimes_{C}\left(K \otimes_{C} \bar{C}\right)=(Y-Z) \otimes_{C}\left(K \otimes_{C} \bar{C}\right)$.

q.e.d.

The quotient field $\mathbf{Q}\left(K \otimes_{C} \bar{C}\right)^{\natural}$ is a field algebraic over $K^{\natural}$ and hence we can find an algebraic closure $\bar{K}^{\natural} \supset \mathbf{Q}\left(K \otimes_{C} \bar{C}\right)^{\natural}$ of $K^{\natural}$. We denote by $q_{\bar{K}}$ the Spec $\bar{K}^{\natural}$-valued point of $Y_{K}^{\natural}$ arising from $q_{K}: \operatorname{Spec}\left(K \otimes_{C} \bar{C}\right)^{\natural} \rightarrow Y_{K}^{\natural}:$ The morphism $q_{\bar{K}}: \operatorname{Spec} \bar{K}^{\natural} \rightarrow \operatorname{Spec}\left(K \otimes_{C} \bar{C}\right)^{\natural} \rightarrow Y_{K}^{\natural}$ is a composite of $q_{K}$ and the morphism defined by the inclusion $\bar{K}^{\natural} \supset \mathbf{Q}\left(K \otimes_{C} \bar{C}\right)^{\natural}$. By applying the Hilbert Nullstellensatz to the fibre of $f: U \rightarrow Y$ over $q_{\bar{K}}\left(\operatorname{Spec} \bar{K}^{\natural}\right)$, we can find a $\bar{K}$ -valued point $\tilde{q}: \operatorname{Spec} \bar{K}^{\natural} \rightarrow U^{\natural} \subset X^{\natural}$ such that $f \circ \tilde{q}=q_{\bar{K}}$. $\tilde{q}: \operatorname{Spec} \bar{K} \rightarrow X^{\natural}$ defines an $\bar{L}^{\natural}$-valued point $\operatorname{Spec} \bar{L}^{\natural} \rightarrow \operatorname{Spec} \bar{K}^{\natural} \rightarrow X^{\natural}$ which we denote by $q$, where $\bar{L}^{\natural}$ is an algebraic closure of $L^{\natural}$ such that $L^{\natural} \supset \bar{K}^{\natural}$.

The morphism $q: \operatorname{Spec} \bar{L}^{\natural} \rightarrow X$ has the following property.

(2.16.1) The image of the $\bar{L}^{\natural}$-valued point $q: \operatorname{Spec} \bar{L}^{\natural} \rightarrow X$ is in $U$ so that we can define $f \circ q: \operatorname{Spec} \bar{L}^{\natural} \rightarrow Y_{K}^{\natural}$.

(2.16.2) The morphism $f \circ q: \operatorname{Spec} \bar{L}^{\natural} \rightarrow Y_{K}^{\natural}$ arises from the Spec $C_{\bar{K}}$-valued point $\varphi:$ Spec $C_{\bar{K}} \rightarrow Y$. More precisely the following diagram is commutative. 


$$
\begin{aligned}
\operatorname{Spec} \bar{L}^{\natural} \stackrel{f \circ q}{\longrightarrow} & Y_{K}^{\natural} \rightarrow Y_{\varphi} \\
\searrow & \downarrow \\
& \operatorname{Spec} \bar{C}_{K}
\end{aligned}
$$

where the morphism $Y_{K}^{\natural} \rightarrow Y$ is the projection and $\operatorname{Spec} \bar{L}^{\natural} \rightarrow \operatorname{Spec} \bar{C}_{K}$ is the morphism induced by the inclusion $\bar{C}_{K} \subset \bar{K}^{\natural} \subset \bar{L}^{\natural}$. It is convenient to denote the composite map of the horizontal map by $\zeta:$ Spec $\bar{L}^{\natural} \rightarrow Y$. On the other hand the inclusion morphism $L^{\natural} \rightarrow \bar{L}^{\natural}$ together with the generic point $\operatorname{Spec} L^{\natural} \rightarrow X$ of $X$ gives a morphism $p: \operatorname{Spec} \bar{L}^{\natural} \rightarrow X^{\natural}$ and a morphism $L \rightarrow \bar{L}^{\natural}[[t]] \rightarrow$ $\bar{L}^{\natural}[[t]]\left[t^{-1}\right]$ of differential fields so that $\bar{L}^{\natural}[[t]]\left[t^{-1}\right]$-valued point $\bar{p}$ : Spec $\bar{L}^{\natural}[[t]]\left[t^{-1}\right] \rightarrow X$ in $\mathscr{F}_{L / K}\left(\bar{L}^{\natural}\right)$ (cf. Remark (2.3.1)). By Proposition (1.4), $q:$ Spec $\bar{L}^{\natural} \rightarrow X^{\natural}$ defines a morphism $\bar{q}$ : Spec $\bar{L}^{\natural}[[t]] \rightarrow X$ of schemes with derivation and hence a morphism $\operatorname{Spec} \bar{L}^{\natural}[[t]]\left[t^{-1}\right] \rightarrow X$ of schemes with derivation composing with inclusion $\bar{L}^{\natural}[[t]] \rightarrow \bar{L}^{\natural}[[t]]\left[t^{-1}\right]$. We denote this morphism again by $\bar{q}: \operatorname{Spec} \bar{L}^{\natural}[[t]]\left[t^{-1}\right] \rightarrow X$. Since $\left(G_{K^{4}}, X^{\natural}\right)$ is a principal homogeneous space, there exists $g \in G\left(\bar{L}^{\natural}\right)$ such that $g p=q$. Thus $g \bar{p}=\bar{q}_{\bar{L}^{4}}$ so that $\bar{q}_{\bar{L}^{\text {q }}}$ is in $\mathscr{F}_{L / K}\left(\bar{L}^{\natural}\right)$. This implies that the image of $\bar{q}:$ Spec $\bar{L}^{\natural}[[t]]\left[t^{-1}\right] \rightarrow X$ is the generic point of $X$. Consequently the image of the morphism $\bar{q}_{\bar{L}^{\natural}}:$ Spec $\bar{L}^{\natural}[[t]] \rightarrow X$ of schemes with derivation contains the generic point of $X$ or the morphism $\bar{q}_{\bar{L}^{4}}$ is dominant. Composing with $f: U \rightarrow Y_{K}$, we get a morphism $\eta: \operatorname{Spec} \bar{L}[[t]] \rightarrow Y_{K}$ of schemes with derivation. Since $Y$ is a scheme with trivial derivation, the morphism $\eta: \operatorname{Spec} \bar{L}^{\natural}[[t]] \rightarrow Y$ of schemes with derivation factors through the morphism Spec $\bar{L}^{\natural}[[t]] \rightarrow \bar{L}^{\natural}$ induced the inclusion $L^{\natural} \subset \bar{L}^{\natural}[[t]]$ so that there exists a morphism $\phi: \operatorname{Spec} \bar{L}^{\natural} \rightarrow Y$ of schemes making the diagram

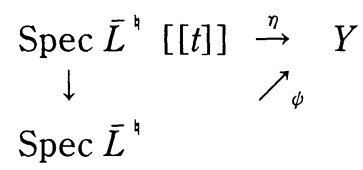

commutative. Necessarily by definition of $\bar{q}, \phi$ coincides with $\zeta$ introduced above. Since $\eta: \operatorname{Spec} \bar{L}^{\natural}[[t]] \rightarrow Y$ is dominant, $\phi=\eta$ is dominant. It follows from the definition that $\eta^{\prime}$ comes from $\bar{q}_{K}$ : Spec $\bar{K}^{\natural} \rightarrow Y$ and hence from $q: \operatorname{Spec} \bar{C} \rightarrow Y$ so that $q$ is dominant. Thus $Y$ is finite over $C_{K}$ and $C_{L}$ is finite over $C_{K}$.

Lemma (2.17). Let $L / K$ be a quasi-automorphic extension with automorphy group $G$ and $M$ the algebraic closure of $K$ in $L$ so that $L \supset M \supset K$. Then the extension $M / K$ is quasi-automorphic with automorphy group $G / G^{0}$. In particular if $G / G^{0}$ is a finite group, then the extension $M / K$ is Galois with Galois group $G / G^{0}$ and hence the quotient group $G / G^{0}$ is uniquely determined by the extension $L / K$. 
Proof. We can find a model $X$ of $L / K$ such that $\left(G_{K^{4}}, X^{\natural}\right)$ is a principal homogeneous space. In particular $X^{\natural}$ is smooth over $K^{\natural}$ so that the local ring $\mathscr{O}_{X^{4}{ }_{x}}$ is a regular local $K^{\natural}$-algebra for every point $x \in X$. Since the regular local ring $\mathscr{O}_{K^{4} x}$ is U.F.D., $\mathscr{O}_{X^{4}{ }_{x}}$ is integrally closed and hence $\mathscr{O}_{X^{4}} \supset M$ for every point $x \in X^{\natural}$. Thus we have the natural $K$-morphism $f: X \rightarrow \operatorname{Spec} M=Y$ of $K$-schemes with derivation. We have a diagram

$$
\begin{array}{rlrl}
G & \times{ }_{C} X \stackrel{\mu}{\rightarrow} & X \\
\operatorname{Id} \times f & \downarrow & & \downarrow{ }^{f} . \\
G & \times{ }_{C} Y & Y
\end{array}
$$

We show that in (2.18) $f \circ \mu$ factors through Id $\times f$. In fact if we go over an algebraic closure $\bar{K}^{\natural}$, then $X \otimes_{K}, \bar{K}^{\natural}$ is a disjoint union of absolutely irreducible components of $X_{\bar{K}^{\natural}}=X \otimes_{K} \bar{K}^{\natural},\left(Y_{\bar{K}}\right)^{\natural}$ is a disjoint union of points isomorphic to Spec $\bar{K}^{\natural}$ of which the number is equal to that of the irreducible components of ( $X$ $\left.\otimes_{K} \bar{K}\right)^{\natural}$ and $f \otimes \operatorname{Id}:\left(X_{\bar{K}}\right)^{\natural} \rightarrow\left(Y_{\bar{K}}\right)^{\natural}$ is the mapping of collapsing each component to a point. Thus once the base change $\bar{K} / K$ is done, $(G \times X)_{\bar{K}}^{\natural} \stackrel{f \circ \mu_{\bar{K}}}{\longrightarrow} Y_{\bar{K}}^{\natural}$ factors through $(\operatorname{Id} \times f) \frac{\natural}{K}:(G \times X) \frac{q}{K} \rightarrow(G \times Y)_{\bar{K}}^{\natural}$. Then it factors already over $K$ if we take care of the following trivial fact.

Sublemma (2.19). Let $E / F$ be a field extension, $f: C \rightarrow A$ an $F$-algebra morphism and $B$ a $F$-subalgebra of $A$. If the image of $f \otimes I d: C \bigotimes_{F} E \rightarrow A \bigotimes_{F} E$ is contained in the subalgebra $B \bigotimes_{F} E$, then the image $f(C)$ is contained in $B$.

Thus we have a morphism $G \times{ }_{C} Y \rightarrow Y$ of the operation of $G$ on $Y$. This morphism is a morphism of $K$-schemes with derivation, where $G \times{ }_{C} Y$ is regarded as a $K$-scheme by a composite of the projection $p_{2}: G \times{ }_{C} Y \rightarrow Y$ and the structure morphism $Y \rightarrow \operatorname{Spec} K$. Hence $G_{\bar{K}}$, operates on $Y^{\natural}$ and the morphism $f$ is $G_{K^{\prime}}$-equivariant. Since $Y$ is finite over $K$, the functor $\mathbf{A u t}_{K^{\prime}} Y^{\natural}$ of the group of automorphisms of $Y^{\natural} / K^{\natural}$ is representable by a finite group scheme. We have a morphism $\varphi: G_{K^{\prime}} \rightarrow \operatorname{Aut}_{K} Y$. The kernel $N$ of $\varphi$ is an algebraic $K^{\natural}$-group scheme. Since $G_{\bar{K}^{\prime}} \simeq X_{\bar{K}^{\prime}}$ and $f_{\bar{K}^{\natural}}: X_{\bar{K}^{4}} \rightarrow Y_{\bar{K}^{\prime}}$ is a morphism of collapsing each component of $X_{\bar{K}}$, to a point, $N_{\bar{K}}=\left(G_{\bar{K}}\right)^{0}$ which coincides with $\left(G^{0}\right)_{\bar{K}}$ by Lemma (1.12). The morphism $G \times{ }_{C} Y \rightarrow Y$ induces the morphism $\bar{\mu}:\left(G / G^{0}\right) \times{ }_{K} Y \rightarrow Y$ of operation of $G / G^{0}$ such that

$$
\left(\bar{\mu}, p_{2}\right):\left(G / G^{0}\right) \times_{C} Y \rightarrow Y \times_{K} Y
$$

is a $K$-morphism of schemes with derivation. The $K$-morphism $\left(\mu, p_{2}\right)$ is an isomorphism since its base change over $\bar{K}$ is an isomorphism. So by Theorem (2.13) 
$M / K$ is quasi-automorphic with automorphy group $G / G^{0}$. If $G / G^{0}$ is a finite group, $M / K$ is Galois such that the Galois group of the extension $M / K$ is isomorphic to $G / G^{0}$ by the argument of the proof of Theorem (2.7).

We can deduce from Theorem (2.13) the following remarkable result.

Theorem (2.21). Let $L / K$ be a quasi-automorphic extension. If $C_{L}=C_{K}$, then the functor $\operatorname{Diff}-\mathbf{b i r}_{K} L:\left(\mathrm{Sch} / C_{K}\right)^{0} \rightarrow(\mathrm{Grp})$ is representable by the automorphy group $G$. In particular under the assumption $C_{L}=C_{K}$, the automorphy group is uniquely determined (cf. Definition (2.23)).

Proof. In fact, let $S$ be a $C=C_{L}=C_{K^{-}}$scheme and $f: X \times{ }_{C} S \cdots \rightarrow X \times{ }_{C}$ $S$ an element of $\mathbf{D i f f}-\mathbf{b i r}{ }_{K} L(S)$ so that $f$ is a $K \otimes_{C} S$-pseudo-automorphism of $X \times{ }_{C} S$ compatible with derivation. We can define an $K \otimes_{C} S$-pseudo-morphism $(f$, Id $): X_{S} \cdots \rightarrow X_{S} \times X_{S}=\left(X \times{ }_{K} X\right)_{S}$ of schemes with derivation, where the fiber product $X_{S} \times X_{S}$ is taken over $K \otimes_{C} S$. Since we have an $X$-isomorphism (2.13.1) of schemes with derivation, we get an $X_{S}$-pseudo-morphism $X_{S} \times X_{S}=$ $\left(X \times_{K} X\right)_{S} \cdots \rightarrow\left(G \times{ }_{C} X\right)_{S}=G_{S} \times_{S} X_{S}$ of schemes with derivation and hence a $K \times{ }_{C} S$-pseudo-morphism $X_{S} \cdots \rightarrow G_{S}$ and hence an $S$-pseudo-morphism $X_{S}$ - $\rightarrow G_{S}$ of schemes with derivation. By Proposition (1.11) the $S$-pseudomorphism $X_{S} \cdots \rightarrow G_{S}$ of schemes with derivation factors through the projection $X_{S} \rightarrow \operatorname{Spec} S$, we get a morphism $\varphi: S \rightarrow G$. In other words, by the isomorphism (2.13.2) $\varphi \operatorname{Id}_{X_{S}}=f$ or the $S$-pseudo-morphism $f: X_{S} \rightarrow X_{S}$ of schemes with derivation is an $S$-morphism $X_{S} \rightarrow X_{S}$ induced by $S \rightarrow G$ form the operation $G \times{ }_{C}$ $X \rightarrow G \times{ }_{C} X$ of $G$ :

$$
\begin{array}{ccc}
S \times{ }_{C} X \rightarrow S \times{ }_{C} X & G \times{ }_{C} X \rightarrow G \times{ }_{C} X \\
\searrow \swarrow & \text { is a pull-back of } & \searrow \swarrow
\end{array}
$$

by the morphism $S \rightarrow G$.

Remark (2.21.1). We show is $\S 3$ that the conditions of Theorem (2.21) are satisfied if and only if $L / K$ is strongly normal in the sense of Kolchin.

Lemma (2.22). If $L / K$ is a quasi-automorphic extension with automorphy group $G$, then $K$ coincides with the field of $G$-invariants of $L$. To be more precise, if $a \in$ $L \backslash K$, then there exists a $C=C_{K^{-}}$scheme $S$ and $g \in G(S)$ such that $g$ defines a $K \otimes_{C} S$-pseudo-automorphism $\phi_{g}: X_{S} \rightarrow X_{S}$ with $\phi_{g}^{*} a \neq a$. Here we regard a as a 
rational function on $X$ and we mean by ${\phi_{g}}^{*} a$ the meromorphic function $a^{\circ} p_{1}{ }^{\circ} \phi_{g}$ on $X_{S}$, $p_{1}: X_{S}=X \times_{c} S \rightarrow X$ being the projection (cf. §4).

Proof. In fact let us take $S=\operatorname{Spec}\left(L \otimes_{K} L\right)^{\natural}$ which is a $K^{\natural}$-scheme. Let $i_{1}$ : $L^{\natural} \rightarrow\left(L \otimes_{K} L\right)^{\natural}$ be the $K^{\natural}$-morphism identifying $L^{\natural}$ with $L^{\natural} \otimes 1$ and $i_{2}: L^{\natural}$ $\rightarrow\left(L \otimes_{K} L\right)^{\natural}$ the $K^{\natural}$-morphism identifying $L^{\natural}$ with $1 \otimes L$ ". We get two morphisms $\quad \bar{i}_{K}: L \rightarrow\left(L \bigotimes_{K} L\right)^{\natural}[[t]] \subset\left(L \otimes_{K} L\right)^{\natural}[[t]]\left[t^{-1}\right], j=1,2$ of differential algebras. So we get two points $q_{i}: \operatorname{Spec}\left(L \otimes_{K} L\right)^{\natural}[[t]]\left[t^{-1}\right] \rightarrow X$ which are morphisms of schemes with derivation. It follows from (2.13.2) there exists $\varphi \in$ $G\left(\left(L \otimes_{K} L\right)^{\natural}\right)$ such that $\varphi q_{1}=q_{2}$. Now by definition if we have $\bar{i}_{1}(b)=\bar{i}_{2}(b)$ for an element $b \in L$, then $b \otimes 1=i_{1}(b)=i_{2}(b)=1 \otimes b$ and hence $b \in K$. Hence $\bar{i}_{1}(a) \neq \bar{i}_{2}(a)$. Now $\varphi$ satisfies our requirement: $\bar{i}_{1}(\varphi(a))=\bar{i}_{2}(a) \neq i_{1}(a)$.

Let $L / K$ be a quasi-automorphic extension with automorphy group $G$. We take the model $X$ with derivation of $L / K$ as in Theorem (2.13). Since the constant field extension $C_{L} / C_{K}$ is finite, as in the Proof of Lemma (2.17), for any $C_{K}$-scheme $S$ the group $G(S)$ operates on the scheme $C_{L} \otimes_{C_{K}} S / S$ of constants by Proposition (1.11). So we have a morphism $\phi: G \rightarrow \mathbf{A u t}_{C_{K}} C_{L}$ of group functors on $\left(\mathrm{Sch} / C_{K}\right)^{0}$. Since the extension $C_{L} / C_{K}$ is finite, the group functor $\operatorname{Aut}_{C_{K}} C_{L}$ is representable by a finite group scheme over $C_{K}$ and hence $N=\operatorname{Ker} \phi$ is a closed subgroup scheme of $G$ such that $G / N$ is a finite group scheme over $C_{K}$.

Let us fix a general notation. Let $f: Y \rightarrow Z$ be a morphism of reduced schemes and $w$ a rational function on $Z$. When we can compose $f$ and $w$ to get a rational function $w^{\circ} f$ on $Y$, the composite function $w^{\circ} f$ will be denoted by $f^{*}(w)$. Let $R$ be a ring of rational functions on $Z$ such that $f^{*}(w)$ is defined for every $w \in R$. Then we have a morphism $f^{*}: R \rightarrow$ (The ring of rational functions on $Y$ ). If $f$ is a morphism of schemes with derivation and if $R$ is closed under the derivation, then $f^{*}$ is a morphism of differential algebras.

Let $L / K$ be a quasi-automorphic extension with automorphy group $G$. We can find a model $X$ as in Theorem (2.13). Let $A \in \operatorname{do}\left(\mathrm{Fld} / K^{\natural}\right)$ and $\varphi_{1}, \varphi_{2} \in$ $\mathscr{F}_{L / K}(A)$. So there exists $h \in G(A)$ such that the diagram

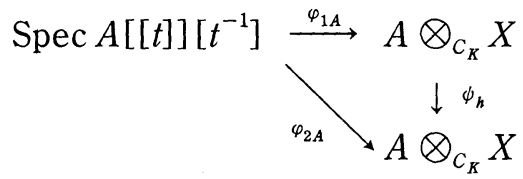

is commutative, where $\varphi_{i A}: \operatorname{Spec} A[[t]]\left[t^{-1}\right] \rightarrow A \otimes_{C_{K}} X$ is a morphism of schemes induced from $\varphi_{i}: \operatorname{Spec} A[[t]]\left[t^{-1}\right] \rightarrow X$ so that the diagram 


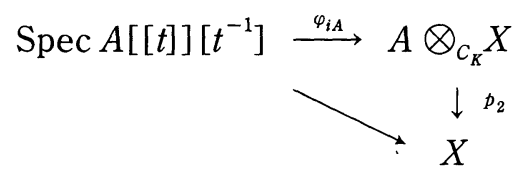

is commutative for $i=1,2$ (cf. Definition (2.5)). In diagram (2.22.1) the morphism $\phi_{h}$ is a induced morphism by the $A$-valued point $h: \operatorname{Spec} A \rightarrow G$. Namely the diagram

$$
\begin{aligned}
& A \otimes_{C_{K}} X \rightarrow G \times_{C_{R}} X \\
& \psi_{k} \quad \downarrow \quad \downarrow \\
& A \otimes_{C_{K}} X \rightarrow G \times_{C_{K}} X
\end{aligned}
$$

is commutative, where the right vertical arrow sends $(g, x)$ to $(g, g x)$. Since we are in characteristic 0 , the schemes in (2.22.1) are reduced. So the notion of a pseudo-function coincides with that of a rational function (cf. E.G.A. Chap. IV, Remarque (20.2.7)). A field $1 \otimes L$ is considered as a field of rational functions on $A \otimes_{C_{K}} X$. Since $\varphi_{1}:$ Spec $A[[t]]\left[t^{-1}\right] \rightarrow X$ factors through the generic point Spec $L \rightarrow X$ of $X$. So by composition with $\varphi_{1}$, we get a morphism

$$
\varphi_{1 A}^{*}: 1 \otimes L \rightarrow A[[t]]\left[t^{-1}\right]
$$

of differential fields. Here we consider $1 \otimes L$ as a field of rational functions on $A \otimes_{C_{K}} X$ and $A[[t]]\left[t^{-1}\right]$ is the field of rational functions on Spec $A[[t]]\left[t^{-1}\right]$ :

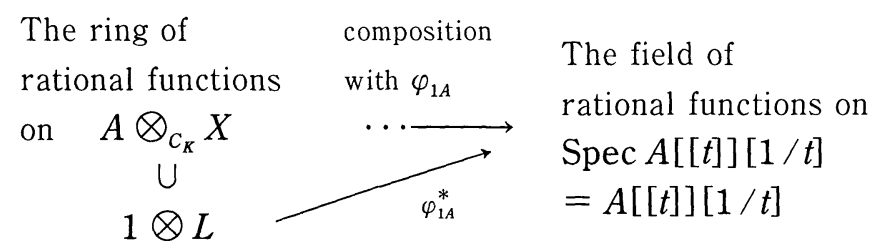

By composition with $\phi_{h}, \phi_{h}^{*}(1 \otimes L)$ is a field of rational functions on $A \otimes_{C_{R}} X$. It follows from diagram $(2.22 .1)$ that we can compose every element in $\phi_{h}^{*}(1 \otimes$ $L)$ with $\varphi_{1 A}$ to get a field of rational functions on $\operatorname{Spec} A[[t]]\left[t^{-1}\right]$ since the morphism $\varphi_{2}$ factors through the generic point of $X$ : In fact for $1 \otimes a, a \in L$, we define $\varphi_{1 A}^{*}\left(\phi_{h}^{*}(1 \otimes a)\right)$ as $\varphi_{2 A}^{*}(1 \otimes a)$. Namely we have a diagram:

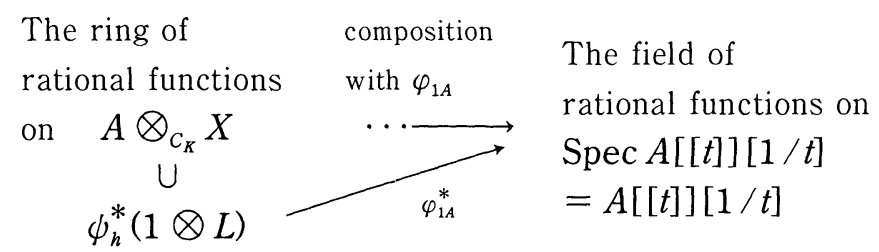


So we get a morphism $\mathbf{Q}\left[1 \otimes L, \phi_{h}^{*}(1 \otimes L)\right] \rightarrow A[[t]]\left[t^{-1}\right]$ of differential rings induced by composition with $\varphi_{1 A}^{*}$ :

$\begin{array}{lll}\begin{array}{l}\text { The ring of } \\ \text { rational functions }\end{array} & \text { composition } \\ \text { with } \varphi_{1 A} & \begin{array}{l}\text { The field of } \\ \text { rational functions on }\end{array} \\ \text { on } A \otimes_{C_{R}} X & \cdots \longrightarrow & \begin{array}{l}\text { Spec } A[[t]][1 / t] \\ =A[[t]][1 / t]\end{array}\end{array}$

Definition (2.23). A quasi-automorphic extension $L / K$ is said to be automorphic if for every $A \in \mathrm{de}\left(\mathrm{Fld} / K^{\mathrm{h}}\right)$ and every $\varphi_{1}, \varphi_{2} \in \mathscr{F}_{L / K}(A)$, the morphism $\varphi_{1 A}^{*}: \mathbf{Q}\left[1 \otimes L, \phi_{h}^{*}(1 \otimes L)\right] \rightarrow A[[t]]\left[t^{-1}\right]$ induced by $\varphi_{1 A}$ is injective.

Proposition (2.24). A Galois extension $L$ of an abstract field $K$ is automorphic.

Proof. Since the automorphic group $G$ is a finite group, every $A$-valued point of $G$ arises from a $K$-valued point of $G$ for any $A \in \operatorname{de}($ Fld $/ K)$. So in particular $h \in G(A)$ comes from an $h^{\prime} \in G(K)$. Hence $\mathbf{Q}\left[\phi_{h}^{*}(1 \otimes L), 1 \otimes L\right]=K\left[\phi_{h}^{*}(1 \otimes\right.$ $L), 1 \otimes L]=K\left[\phi_{h^{\prime}}^{*}(1 \otimes L), 1 \otimes L\right]=K[1 \otimes L]=1 \otimes L$ and the map in Definition (2.23) is injective.

We can slightly generalize Proposition (2.24).

Proposition (2.25). Let $L$ be a quasi-automorphic extension of an abstract field $K$ with automorphy group $G$.

(i) Let $F$ be an abstract field extension. If $L \bigotimes_{K} F$ is a field, then $L \bigotimes_{K} F / F$ is quasi-automorphic with automorphy group $G_{F}$.

(ii) Let $F$ be an abstract field extension of $K$ satisfying the condition (i). The following conditions are equivalent: (a) The extension $L \bigotimes_{K} F / F$ is Galois with Galois group isomorphic to $G_{F}$; (b) The group scheme $G_{F}$ is a finite group.

(iii) If there exists a field extension $F / K$ satisfying these equivalent conditions in (ii), then the extension $L / K$ is automorphic.

Proof. (i) follows from Theorem (2.7). The equivalence of the conditions in (ii) is well-known. Assume that there exists a field extension $F / K$ satisfying the equivalent conditions of (ii). Let $A \in \operatorname{ab}(\mathrm{Fld} / K)$ and $\varphi_{1}, \varphi_{2} \in \mathscr{F}_{L / K}(A)$ as in Definition (2.23). Since the extenpion $L / K$ is finite by Theorem (2.7), $X=\operatorname{Spec} L$ is the unique model of $L / K$. We can find a field $A^{\prime} / K$ that contains two fields 
$K$-isomorphic to $A$ and $F$ respectively. We have natural inclusions $\mathscr{F}_{L / K}(A) \subset$ $\mathscr{F}_{L / K}\left(A^{\prime}\right) \subset \mathscr{F}_{L \otimes_{K} F / F}\left(A^{\prime}\right)$ so that there correspond to $\varphi_{1}, \varphi_{2} \in \mathscr{F}_{L / K}(A)$ elements $\varphi_{1}^{\prime}, \varphi_{2}^{\prime} \in \mathscr{F}_{L \otimes F / F}\left(A^{\prime}\right)$. In fact $\varphi_{1}^{\prime}, \varphi_{2}^{\prime} \in \mathscr{F}_{L \otimes F / F}\left(A^{\prime}\right)$ are defined as follows. Since the field $A^{\prime}$ contains $F$, the composite morphism Spec $A^{\prime}[[t]]\left[t^{-1}\right] \rightarrow$ Spec $A[[t]]\left[t^{-1}\right] \stackrel{\varphi_{i}}{\rightarrow} \operatorname{Spec} L=X$ factors through the first projection $p_{1}: \operatorname{Spec} L \bigotimes_{K} F=$ $X \otimes_{K} F \rightarrow X$ so that we have a morphism $\varphi_{i}^{\prime}: \operatorname{Spec} A^{\prime}[[t]]\left[t^{-1}\right] \rightarrow X_{F}$ of schemes with derivation making the following diagram commutative.

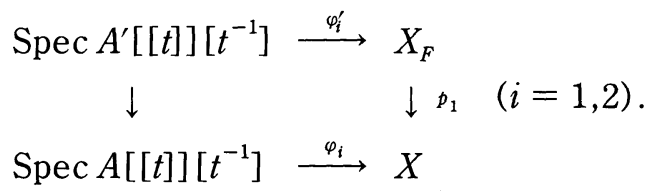

We can regard the $A$-valued point $h \in G(A)$ with $\varphi_{2}=h \varphi_{1}$ as an $A^{\prime}$-valued point $h^{\prime} \in G\left(A^{\prime}\right)$. The point $h^{\prime}$ induces an $A^{\prime}$-automorphism $\phi_{h^{\prime}}: A^{\prime} \bigotimes_{K} X \rightarrow A^{\prime}$ $\bigotimes_{K} X$, that is, an $A^{\prime}$-automorphism of $A^{\prime} \bigotimes_{F} X_{F}$ such that $\varphi_{2}^{\prime}=\phi_{h^{\prime}}{ }^{\circ} \varphi_{1}^{\prime}$. Since $L$ $\otimes_{K} F / F$ is Galois,

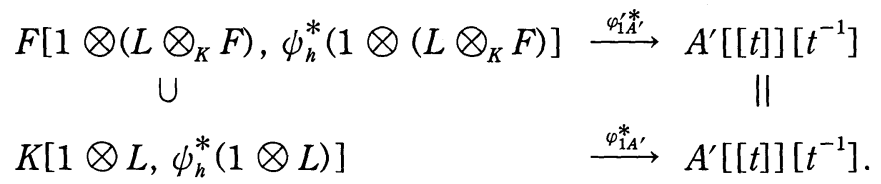

In $[G]$ they introduced the notion of an almost classically Galois extension and proved the Galois correspondence for a such extension. Namely a quasiautomorphic extension $L$ of an abstract field $K$ with automorphy group $G$ is almost classically Galois if there exists a Galois extension $F / K$ such that $L \otimes_{K}$ $F / F$ is a Galois extension and such that $G \bigotimes_{K} F$ is its Galois group. So an almost classically Galois extension is a particular case of the extension in Proposition (2.25.iii).

COROLlary (2.26). An almost classically Galois extension in the sense of $[G]$ is automorphic.

In [G] they proved that the Galois correspondece exists for an almost classically Galois extension, which is one of their main results (5.2 Theorem in $[G]$ ). We show in $\S 4$ Theorem (4.10) that the Galois correspondence exists for an automorphic extension. So the result of $[G]$ follows from Corollary (2.26) and Theorem (4.10).

Proposition (2.27). A quasi-automorphic extension $L / K$ with $C_{L}=C_{K}$ is automorphic. 
Proof. Let $A \in$ ob (Fld $\left./ K^{\natural}\right)$ and $X$ be a model on which the automorphy group $G$ operates. Let $\varphi_{1}, \varphi_{2} \in G(A)$ and $h \in G(A)$ as in Definition (2.23). We have a morphism Spec $A[[t]]\left[t^{-1}\right] \stackrel{\varphi_{1 A}}{\longrightarrow} A \otimes_{C_{K}} X$. Since $C_{K}=C_{L}, L / C_{K}$ is a regular extension so that $A \otimes_{C_{K}} L$ is an integral domain. We have $\varphi_{1 A}^{*}: A \bigotimes_{C_{K}} L \rightarrow$ $A\left[[t]\left[t^{-1}\right]\right.$. It is sufficient to show that this map is injective. The intersection $\varphi_{1 A}^{*}(1 \otimes L) \cap A$ in $A[[t]]\left[t^{-1}\right]$ is $C_{\varphi_{1 A}^{*}(1 \otimes L)}=\varphi_{1 A}^{*}\left(1 \otimes C_{L}\right)=\varphi_{1 A}^{*}\left(1 \otimes C_{K}\right)=C_{K}$ $\left(\subset K^{\natural} \subset A[[t]]\left[t^{-1}\right]\right.$ ) since $A$ is the field of constants of $A[[t]]\left[t^{-1}\right]$. Hence by Lemma (1.1) $\varphi_{1 A}^{*}(1 \otimes L)$ and $A$ are linearly disjoint over $\varphi_{1 A}^{*}\left(1 \otimes C_{K}\right)$. So the mapping in question induced by $\varphi_{1 A}$ is injective. This is what we had to show.

\section{§3. Relation with the Kolchin theory}

The differential Galois theory of Kolchin depends on the language of algebraic geometry of Weil. Namely let $\Omega$ be a differential universal domain so that $\Omega$ is a differential field and all the differential fields which he considers are differential subfields of $\Omega$. The differential universal domain $\Omega$ is fixed once for all and he studies small differential subfields of $\Omega$. We mean by a small differential subfield of $\Omega$ a differential subfield $K$ of $\Omega$ such that for every differentially finitely generated field extension $L$ of $K$, there exists a differential $K$-embedding $f: L \rightarrow \Omega: f$ is a morphism of differential fields such that $f(a)=a$ for every $a \in K$.

Let $L / K$ be a differential field extension which satisfies the finiteness condition (CF) of $\S 1$. He says that a $K$-morphism $f: L \rightarrow \Omega$ of differential fields is strong if (i) $f(a)=a$ for every constant $a \in L$ and if (ii) the composite field $f(L)$. $L$ which is a differential field is generated over $L$ by constants. He says that the extension $L / K$ is strongly normal if every $K$-morphism $L \rightarrow \Omega$ is strong.

Remark (3.1). It follows from Corollary (1.2) that the following conditions are equivalent.

(i) The composite field $f(L) L$ is generated over $L$ by constants.

(ii) $f(L)$ is contained in the composite field $f(L) C_{\Omega}$.

Example (3.2). Let us analyze Example (2.1): $K=\mathbf{C}(x), L=\mathbf{C}(x, \exp x)$ with derivation $d / d x$. Any morphism $f: L \rightarrow \Omega$ of differential field is strong. In fact, since $y=\exp x$ is a solution of the differential equation (2.1.1), the image $f(y)=z$ satisfies the differential equation

$$
z^{\prime}=z
$$

Then it follows from (2.1.1) and (3.2.1) by the argument of Example (2.1) that 
$y z^{-1}$ is a constant so that $f(L) L=K(y, z)=K\left(y, y z^{-1}\right)$ is generated over $L=$ $K(y)$ by the constant $y z^{-1}$. Here we notice that we may replace $\mathbf{C}$ by any abstract field, for example $\mathbf{Q}$.

As is widely recognized, it is more convenient to avoid the universal domain. We adopt the following

Definition (3.3). Let $L / K$ be a differential field extension satisfying the finiteness condition (FC) of $\S 1$.

(1) Let $f, g: L \rightarrow M$ be two $K$-morphisms of differential field extensions of $K$. We say that the morphism $f$ is strong over $g$ if (i) $f(a)=g(a)$ for every constant $a$ of $L$ and if (ii) the composite field $f(L) . g(L)$ is generated over $g(L)$ by constants.

(2) We say that the extension $L / K$ is strongly normal if for every differential field extension $M$ of $K$ and for arbitrary two $K$-morphisms $f, g: L \rightarrow M$ of differential fields, $f$ is strong over $g$.

Remark (3.4). As we noticed above, the condition (3.3.2) is satisfied if and only if the image $f(L)$ is contained in the composite field $g(L) C_{M}$.

Kolchin uses the case where $M$ is the universal domain $\Omega$ and $g: L \rightarrow \Omega$ is the identity map. Evidently our Definition (3.2) of strongly normal extension coincides with Kolchin's. In fact it is sufficient to notice the following: For every differential field extension $N_{1} / K$ satisfying the finiteness condition (CF) of $\S 1$, every differential intermediate field $N_{1} \supset N_{2} \supset K$ and for any morphism $\varphi: N_{2} \rightarrow \Omega$ of differential fields, the morphism $\varphi$ can be extended to a morphism $\tilde{\varphi}: N_{1} \rightarrow \Omega$ of differential fields.

Let $L / K$ be a differential field extension satisfying the finiteness condition (CF) of $\S 1$. As is explained in $\S 1$, the total quotient ring $\mathbf{Q}\left(L \bigotimes_{K} L\right)$, which is a differential ring, is a direct product of the differential fields $M_{t}: \mathbf{Q}\left(L \bigotimes_{K} L\right)=$ $\Pi_{i=1}^{n} M_{i}$.

Lemma (3.5). Let $L / K$ be a differential field extension satisfying the finiteness condition (CF) of $\S 1$ and $a \in L \backslash K$. Then there exist K-morphisms $f, g: L \rightarrow M$ of $K$-differential fields such that $f(a) \neq g(a)$.

Proof. We use the notation of $\S 1$ below Corollary (1.12.1). Since $a \otimes 1 \neq 1$ $\otimes a$ in $L \bigotimes_{K} L \subset \mathbf{Q}\left(L \bigotimes_{K} L\right)=\prod_{i=1}^{n} M_{i}$, there exists a differential field $M_{i}$ such 
that for the $i$-th projection $f_{i}: \mathbf{Q}\left(L \otimes_{K} L\right) \rightarrow M_{\imath} f_{i}(a \otimes 1) \neq f_{i}(1 \otimes a)$.

Lemma (3.6). If an extension $L / K$ is strongly normal, then the field of constants $C_{L}$ of $L$ coincides with the field of constants $C_{K}$ of $K$.

Proof. If $a \in L \backslash K$, then by Lemma (3.5) there exist $K$-morphisms $f, g$ : $L \rightarrow M$ of $K$-differential fields such that $f(a) \neq g(a)$. Since $f$ is strong over $g, a$ is not constant by Definition (3.3. i). Namely any constant in $L$ is contained in $K$ so that $C_{L} \subset C_{K}$. The opposite inclusion being trivial, the Lemma is proved.

Definition (3.7). Let $L / K$ be a differential field extension satisfying the finiteness condition (CF) of $\S 1$ and $f, h_{1}: L \rightarrow M_{1}, g, h_{2}: L \rightarrow M_{2}$ four $K$ morphisms of $K$-differential fields. We say that the morphism $\left(g, h_{2}\right)$ is a specialization of $\left(f, h_{1}\right)$ or simply $g$ is a specialization of $f$ if there exists a differential algebra morphism $r: \mathbf{Q}\left[h_{1}(L), f(L)\right] \rightarrow \mathbf{Q}\left[h_{2}(L), g(L)\right]$ making the diagram below commutative:

$$
\begin{array}{cc}
L \otimes_{K} L \stackrel{p}{\rightarrow} & \mathbf{Q}\left[h_{1}(L), f(L)\right] \subset M_{1} \\
{ }_{q}^{\searrow} & \\
& \mathbf{Q}\left[h_{2}(L), g(L)\right] \subset M_{2}
\end{array}
$$

where $p, q$ are the natural morphisms induced from $f, g$ respectively: $p(a \otimes b)=$ $f(a) h_{1}(b), q(a \otimes b)=g(a) h_{2}(e)$ for $a \in L$ and $b \in L$.

Remark (3.7.2). If we regard the fields $M_{1}$ and $M_{2}$ as over fields of $L$ respectively $h_{1}$ and $h_{2}$, and $L \bigotimes_{K} L$ as an $L$-algebra by $a \mapsto 1 \otimes a$, then the morphisms $p, q$ are $L$-morphisms and the commutativity of the diagram (3.7.1) requires that the morphism $r$ is an $L$-morphism.

Lemma (3.8). If $\left(f, h_{2}\right)$ is a specialization of $\left(g, h_{1}\right)$ and $f$ is strong over $h_{1}$, then $g$ is strong over $h_{2}$.

Proof. We need the following well-known fact (see for example [K1], Chap. II, $\S 1$, Theorem 1).

Sublemma (3.8.1). The following two conditions for elements $y_{1}, y_{2}, \ldots, y_{n}$ of a differential field $K$ are equivalent.

(1) $y_{1}, y_{2}, \ldots, y_{n}$ are linearly independent over the field $C_{K}$ of constants of $K$;

(2) The Wronskian $W\left(y_{1}, y_{2}, \ldots, y_{n}\right) \neq 0$ (Let us recall that the Wronskian 
$W\left(y_{1}, y_{2}, \ldots, y_{n}\right)$ is the determinant of a matrix $\left.\left(y_{j}^{(2-1)}\right)_{1 \leq i, j \leq n}\right)$

If we regard $L \bigotimes_{K} L$ as an $L$-algebra by identifying $L$ with $1 \otimes L \subset L \bigotimes_{K} L$ and $M_{1}, M_{2}$ respectively by $h_{1}, h_{2}$, then the morphisms in diagram (3.7.1) are $L$-morphisms (cf. Remark (3.7.2)). It follows from Remark (3.4) that we have to show that $g(L) \subset h_{2}(L) C_{M_{2}}$. We show $g(a) \in h_{2}(L) C_{M_{2}}$ for $a \in L$. Let $\left\{e_{i}\right\}_{i \in I}$ be a basis of the $C_{L^{-}}$vector space $L$. Since $f$ is strong over $h_{1}$. we have $f(L) \subset$ $h_{1}(L) C_{M_{1}}$ so that we can write

$$
f(a)=\sum_{i} a_{i} h_{1}\left(e_{i}\right) / \sum_{i} b_{i} h_{1}\left(e_{i}\right) \text { with } a_{i}, b_{i} \in C_{M_{1}}
$$

for all $i \in I$. Here almost all the $a_{\imath}$ and $b_{i}$ are equal to zero. We get from (3.8.2)

$$
\sum_{i} b_{i} f(a) h_{1}\left(e_{i}\right)-\sum_{i} a_{i} h_{1}\left(e_{i}\right)=0 .
$$

In particular a finite number of the $f(a) h_{1}\left(e_{1}\right)$ and $h_{1}\left(e_{i}\right)$ are linearly dependent over $C_{M_{1}}$. Say $f(a) h_{1}\left(e_{i_{l}}\right), h_{1}\left(e_{i_{l}}\right) 1 \leq l \leq n$ are linearly dependent over $C_{M_{1}}$. By Sublemma (3.8.1), $W\left(f(a) h_{1}\left(e_{i_{1}}\right), f(a) h_{1}\left(e_{i_{2}}\right), \ldots, f(a) h_{1}\left(e_{i_{n}}\right), h_{1}\left(e_{i_{1}}\right), h_{1}\left(e_{i_{2}}\right), \ldots\right.$, $\left.h_{1}\left(e_{i_{n}}\right)\right)=0$. Since $W$ is a differential polynomial of the $f(a) h_{1}\left(e_{i_{l}}\right)$ and $h_{1}\left(e_{i_{l}}\right)$ $1 \leq l \leq n$, with coefficients in $\mathbf{Q}$, we get $0=W\left(r\left(f(a) h_{1}\left(e_{i_{1}}\right)\right), r\left(f(a) h_{1}\left(e_{i_{2}}\right)\right), \ldots\right.$, $r\left(f(a) h_{1}\left(e_{i_{n}}\right)\right), r\left(h_{1}\left(e_{i_{1}}\right)\right), r\left(h_{1}\left(e_{i_{2}}\right)\right), \ldots, r\left(h_{1}\left(e_{i_{n}}\right)\right)=W\left(g(a) h_{2}\left(e_{i_{1}}\right), g(a) h_{2}\left(e_{i_{2}}\right)\right.$, $\left.\ldots, g(a) h_{2}\left(e_{i_{n}}\right), h_{2}\left(e_{i_{1}}\right), h_{2}\left(e_{i_{2}}\right), \ldots, h\left(e_{i_{n}}\right)\right)$. So $g(a) h_{2}\left(e_{i_{1}}\right), g(a) h_{2}\left(e_{i_{2}}\right), \ldots, g(a)$ $h_{2}\left(e_{i_{n}}\right), h_{2}\left(e_{i_{1}}\right), h_{2}\left(e_{i_{2}}\right), \ldots, h_{2}\left(e_{i_{n}}\right)$ are linearly dependent over $C_{M_{2}}$ by Sublemma (3.8.1): We can find a non-trivial linear relation

$$
\sum_{i} \beta_{1} g(a) h_{2}\left(e_{i}\right)-\sum_{i} \alpha_{i} h_{2}\left(e_{i}\right)=0
$$

where $\alpha_{i}, \beta_{i}$ are in $C_{M_{2}}$ and equal to 0 except for a finite number of indices. We have either (i) every $\beta_{i}$ is equal to 0 or (ii) one of the $\beta_{i}$ is not equal to 0 . We show that the first case never occurs. If the first case happened, it would follow from (3.8.3)

$$
\sum_{i} \alpha_{i} h_{2}\left(e_{i}\right)=0
$$

Since $e_{i}, i \in I$ are linearly independent over $C_{L}$, the $h_{2}\left(e_{i}\right), i \in I$ are linearly independent over $h_{2}\left(C_{L}\right)=C_{h_{2}(L)}$. On the other hand $C_{M_{2}}$ and $h_{2}(L)$ are linearly disjoint over $h_{2}\left(C_{L}\right)=C_{h_{2}(L)}$ by Lemma (1.1) so that the $h_{2}\left(e_{\imath}\right), i \in I$ are linearly independent over $C_{M_{2}}$. Hence (3.8.4) would imply that $\alpha_{i}=0$ for every $i \in I$. Consequently $\alpha_{i}=\beta_{i}=0$ for every $i \in I$. This is a contradiction. So the latter case always occurs: At least one of the $\beta_{i}$ is not equal to 0 . As we have seen above, the $h_{2}\left(e_{i}\right)$ are linearly independent over $C_{M_{2}}$ so that $\sum_{i} \beta_{i} g(a) h_{2}\left(e_{i}\right) \neq 0$ and $g(a)=$ 
$\sum_{i} \alpha_{i} h_{2}\left(e_{i}\right) / \sum_{i} \beta_{i} h_{2}\left(e_{i}\right)$. Thus $g(a) \in h_{2}(L) C_{M}$.

Lemma (3.9). For a differential field extension $L / K$ satisfying the finiteness condition (C.F) of $\S 1$, the following conditions (1) and (2) are equivalent.

(1) The extension $L / K$ is strongly normal (cf. Definition (3.3)).

(2) The field of constants $C_{L}$ of $L$ coincides with the field $C_{K}$ of $K$. We using the notation of $\S 1$ below Corollary (1.12.1), the composite fields $L_{i}^{(1)}, L_{i}^{(2)}$ in $M_{i}$ are generated over $L_{i}^{(2)}$ by constants for $1 \leq i \leq n$. Namely the morphisms $f_{i}^{1}$ is strong over $f_{i}^{2}$ for $1 \leq i \leq n$.

Proof. If the extension $L / K$ is strongly normal, then $C_{L}=C_{K}$ by Lemma (3.6). Since we have the two $K$-morphisms $f_{i}^{1}, f_{i}^{2}: L \rightarrow M_{i}$ of differential fields such that $f_{i}^{1}(L)=L_{i}^{(1)}, f_{i}^{2}(L)=L_{i}^{(2)}$ for $1 \leq i \leq n$, the condition (1) implies the condition (2) by definition.

Let us now assume that the condition (2) is satisfied. Let $f, g: L \rightarrow M$ be two $K$-morphisms of differential fields so that we get an $L$-morphism $h: L \bigotimes_{K} L \rightarrow M$ of differential $L$-algebras such that $h(a \otimes b)=f(a) g(b)$ for $a, b \in L$. Here we regard $L \bigotimes_{K} L, M$ as $L$-algebras respectively by $L \rightarrow 1 \otimes L \subset L \bigotimes_{K} L, g: L \rightarrow$ $M$. Then $h$ factors through one of the $M_{i}$. Namely let us regard $M_{\imath}$ as an $L$-algebra by $f_{i}^{2}: L \rightarrow M_{i}$ and set $A_{i}=\mathbf{Q}\left[f_{i}^{2}(L), f_{i}^{1}(L)\right]=f_{i}\left(L \bigotimes_{K} L\right)$ so that $A_{i}$ is a differential $L$-subalgebra of $M_{i}$. Then there exists a differential $L$-morphism $\varphi: A_{\imath} \rightarrow M$ such that $\left.\varphi \circ f_{i}\right|_{L \otimes_{K} L}=h$, where $f_{i}: L \otimes_{K} L \subset \mathbf{Q}\left(L \otimes_{K} L\right)=\Pi_{i=1}^{n} M_{i}$ $\rightarrow M_{i}$ is the projection as in $\S 1$. In fact let $\mathscr{L}$ be an abstract $K^{\natural}$-subalgebra of $L$ of finite type over $K$ so that an algebra $\mathscr{L} \otimes_{K^{*}} L^{\natural}$ is of finite type over $L^{\natural}$. Then $h_{\mathscr{L}}=h \mid \mathscr{L} \otimes_{K^{\prime}} L^{\natural}: \mathscr{L} \otimes_{,} L^{\natural} \rightarrow M$ is an $L^{\natural}$-morphism of algebras so that the image of the morphism ${ }^{a} h_{\mathscr{L}}: \operatorname{Spec} M^{\natural} \rightarrow \operatorname{Spec}\left(\mathscr{L} \bigotimes_{K^{*}} L^{\natural}\right)$ lies on an irreducible component, say the $i$-th irreducible component $X_{i}$ of the algebraic $L^{k}$-scheme $\operatorname{Spec}\left(\mathscr{L} \otimes_{K}, L^{\natural}\right)$. Hence the image point ${ }^{a} h_{\mathscr{L}}(\operatorname{Spec} M)$ is a specialization of the generic point of $L^{\natural}$-integral scheme $X_{i}$. Namely if we set $A_{\mathscr{L}}=\mathbf{Q}\left[f_{1}^{2}(L)\right.$, $\left.f_{i}^{1}(\mathscr{L})\right]=f_{i}\left(\mathscr{L} \bigotimes_{K} L\right)$, then there exists an $L^{\natural}$-morphism $\varphi_{\mathscr{L}}: A_{\mathscr{L}} \rightarrow M^{\natural}$ of algebras making the diagram

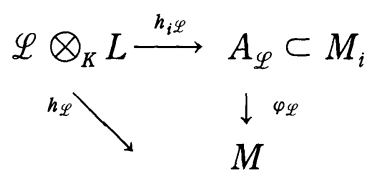

commutative, where we set $f_{i \mathscr{L}}=f_{i} \mid \mathscr{L} \otimes_{K} L$ so that we have $f_{1 \mathscr{L}}(a \otimes b)=$ $f_{i}^{(1)}(a) f_{i}^{(2)}(b)$ for $a \in \mathscr{L}, b \in L$. Since the subalgebra $A_{\mathscr{L}}$ and the morphisms $f_{i \mathscr{Q}}$, $h_{\mathscr{L}}, \varphi_{\mathscr{L}}$ are uniquely determined by the subalgebra $\mathscr{L}$, we can glue them: If $\mathscr{L}^{\prime}$ be 
another abstract $K^{\natural}$-subalgebra of $L^{\natural}$ of finite type over $K^{\natural}$, then $f_{i \mathscr{L}}$ and $f_{i \mathscr{Q}^{\prime}}$, $h_{\mathscr{L}}$ and $h_{\mathscr{L}^{\prime}}, \varphi_{\mathscr{L}}$ and $\varphi_{\mathscr{L}^{\prime}}$ coincide when restricted on the subsets where the both morphisms are defined. In this way the morphisms $f_{i \mathscr{L}}$ and $h_{\mathscr{L}}$ recover respectively $\left.f_{i}\right|_{L} \otimes_{K} L$ and $h$ and the morphism $\varphi_{\mathscr{L}}$ defines an abstract algebra morphism $\varphi$ : $\mathbf{Q}\left[f_{i}^{2}\left(L_{1}\right), f_{i}^{1}(L)\right]=A_{\imath} \rightarrow \mathbf{Q}[g(L), f(L)]$ making the diagram

$$
\begin{aligned}
L \otimes_{K} L \stackrel{p}{\rightarrow} \mathbf{Q}\left[f_{i}^{1}(L), f_{i}^{2}(L)\right]=A_{\imath} \subset M_{\imath} \\
h \backslash \quad \\
\\
\mathbf{Q}[g(L), f(L)]
\end{aligned}
$$

commutative so that we have

$$
\varphi\left(f_{i}^{1}(a) f_{i}^{1}(b)\right)=f(a) g(b) \text { for } a, b \in L .
$$

Here we denote $\left.f_{i}\right|_{L \otimes_{K} L}$ by $p$. Since the canonical morphisms $f_{i}^{(1)}(L) \rightarrow f(L)$, $f_{i}^{(2)}(L) \rightarrow g(L)\left(f_{i}^{(1)}(a) \mapsto f(a), f_{i}^{(2)}(a) \mapsto g(a)\right.$ for $\left.a \in L\right)$ are differential morphisms, the morphism $\varphi$ is a differential morphism by (3.9.1). In other words $(f, g)$ is a specialization of $\left(f^{(1)}, f^{(2)}\right)$ so that $f$ is strong over $g$ by Lemma (3.8). Hence the condition (1) is satisfied.

THEOREM (3.10). For a differential field extension $L / K$ satisfying the finiteness condition (FC) of $\S 1$ the following conditions (1) and (2) are equivalent.

(1) The extension $L / K$ is quasi-automorphic and the field $C_{L}$ of constants of $L$ coincides with the field $C_{K}$ of constants of $K$ (so that $L / K$ is automorphic by Proposition (2.27)).

(2) The extension $L / K$ is strongly normal (cf. Definition (3.2)).

Proof. We use the notation of $\S 1$ below Corollary (1.12.1). We assume that the extension $L / K$ is quasi-automorphic. We have two $K$-morphisms $f_{i}^{1}$ and $f_{i}^{2}$ of differential fields: $f_{i}^{1}, f_{i}^{2}: L \rightarrow M_{1}$ for $1 \leq i \leq n$. In particular we have morphisms $f_{i}^{1{ }^{\natural}}, f_{i}^{2 \natural}: L^{\natural} \rightarrow M^{\natural}$ of fields and hence by Remark (2.3.1) we get two morphisms $\bar{f}_{i}^{1}, \bar{f}_{i}^{2}: L \rightarrow M_{1}^{\natural}[[t]] \subset M_{i}^{\natural}[[t]]\left[t^{-1}\right]$ of differential algebras which are in $\mathscr{F}_{L / K}\left(M_{i}^{\natural}\right)$. Therefore there exists $g_{i} \in G\left(M_{i}^{\natural}\right)$ such that $g_{i} \bar{f}_{i}^{1}=\bar{f}_{i}^{2}$. This shows in particular that we denoting by $N_{i}$ a composite field of $\bar{f}_{i}^{1}(L)$ and $\bar{f}_{i}^{2}(L)$ in $M_{i}[[t]]\left[t^{-1}\right]$, then $N_{i}$ is generated over $\bar{f}_{i}^{2}(L)$ by constants. Since $i\left(L_{\imath}^{(1)}\right)=\bar{f}_{\imath}^{1}(L)$ and $i\left(L_{i}^{(2)}\right)=\bar{f}_{i}^{2}(L)$ by definition, we can conclude that $\mathbf{Q}\left(L_{i}^{(1)} . L_{2}^{(2)}\right)$ is generated over $L_{\imath}^{(2)}$ by constants, where $i: M_{\imath} \rightarrow M_{\imath}^{\natural}[[t]] \subset$ $M_{\imath}^{\natural}[[t]]\left[t^{-1}\right]$ is the universal Taylor morphism and canonical inclusion. It follows from Lemma (3.9) that the extension $L / K$ is strongly normal.

Let us now assume that the extension is strongly normal. We show that the 
total quotient ring $\mathbf{Q}\left(L \bigotimes_{K} L\right)=\prod_{i=1}^{n} M_{i}$ of $L \bigotimes_{K} L$ is generated over $1 \otimes L$ by constants. More precisely the ring $\mathbf{Q}\left(L \bigotimes_{K} L\right)$ is a quotient ring of a subring of $\mathbf{Q}\left(L \otimes_{K} L\right)$ generated over $1 \otimes L$ by constants. To this end it is sufficient to prove that the subring $L \otimes 1$ of $\mathbf{Q}\left(L \otimes_{K} L\right)$ is contained in the total quotient ring $S$ of a ring generated by $1 \otimes L$ and the constants of $\mathbf{Q}\left(L \otimes_{K} L\right)$, where we consider the ring $S$ as a subring of $\mathbf{Q}\left(L \bigotimes_{K} L\right)$. The idempotents $e_{i}$ giving the decomposition $\mathbf{Q}\left(L \otimes_{K} L\right)=\Pi_{i=1}^{n} M_{i}$ are constants by Corollary (1.11.1) so that

$$
S \supset(1 \otimes L) e_{i}=L_{i}^{(2)} \text { for } 1 \leq i \leq n .
$$

Since in $M_{i}$ a composite field $L_{i}^{(1)} L_{i}^{(2)}$ is generated over $L_{i}^{(2)}$ by constants, it follows from (3.10.1) $S \supset L_{\imath}^{(1)} L_{\imath}^{(2)} \supset L_{i}^{(1)}=(L \otimes 1) e_{\imath}$. Hence $S \supset \sum_{i=1}^{n} L_{1} e_{i} \supset L \otimes 1$. This is what we wanted to show. It follows from Corollary (1.11.1) that the ring of constants $C_{\left.\mathbf{Q}^{(L} \otimes_{K} L\right)}$ is the direct product of the fields $C_{M_{i}}$ which are finitely generated over $C_{K}=C_{L}$. Let $G^{\prime}$ be a model of $C_{\left.\mathbf{Q}^{(L} \otimes_{K} L\right)} / C$. Namely $G^{\prime}$ is a reduced and noetherian $C_{K^{\prime}}$ scheme over $C$ whose ring $C\left(G^{\prime}\right)$ of rational functions (or the total quotient ring of the ring $C\left[G^{\prime}\right]$ of regular functions if we choose an affine model $\left.G^{\prime}\right)$ is isomorphic to $C_{\left.\mathbf{Q}^{(L} \otimes_{K} L\right)}$. We notice that $C\left(G^{\prime}\right)$ is also isomorphic to $\Pi_{x} \mathscr{O}_{G^{\prime} x}$, where the $x$ are minimal points of $G^{\prime}$. It follows from Lemma (1.1) that in $\mathbf{Q}\left(L \otimes_{K} L\right)$, the field $1 \otimes L$ and $C_{\left.\mathbf{Q}^{(L} \otimes_{K} L\right)}$ are linearly disjoint over $C$. So $\mathbf{Q}\left(L \otimes_{K} L\right)$ is differentially $L$-isomorphic to the total quotient $\operatorname{ring} \mathbf{Q}\left(C_{\left.\mathbf{Q}^{(L} \otimes_{K} L\right)}\right.$ $\left.\otimes_{C} L\right):$ We have a differential isomorphism $\theta: \mathbf{Q}\left(L \otimes_{K} L\right) \rightarrow \mathbf{Q}\left(C_{\left.\mathbf{Q}^{(L} \otimes_{K} L\right)} \otimes_{C} L\right)$ such that $\theta(1 \otimes L)=1 \otimes L$. Composing $\theta$ with the inclusion $L \rightarrow \mathbf{Q}\left(L \otimes_{K} L\right)$, $a \mapsto a \otimes 1$ for $a \in L$, we get a differential morphism $L \rightarrow \mathbf{Q}\left(L \otimes_{K} L\right) \rightarrow$ $\mathbf{Q}\left(C_{\left.\mathbf{Q}^{(L} \otimes_{K} L\right)} \otimes_{C} L\right)$. In the language of schemes, we have a $K$-birational maps compatible with derivations $G^{\prime} \times_{C} X \cdots \rightarrow X \times_{K} X$ and $\mu^{\prime}: G^{\prime} \times_{C} X \cdots \rightarrow X$ such that the first map $G^{\prime} \times{ }_{C} X \cdots \rightarrow X \times{ }_{K} X$ is nothing but $\left(\mu^{\prime}, p_{2}\right)$. Hence we have a $G^{\prime}$-rational map $\left(\mathrm{Id}, \mu^{\prime}\right): G^{\prime} \times_{C} X \cdots \rightarrow G^{\prime} \times_{C} X$.

Sublemma (3.10.2). Let $S$ be a reduced noetherian $C$-scheme and $n: S \times{ }_{c} X$ $\cdots \rightarrow X$ be a $K$-pseudo-morphism compatible with derivation such that the rational map $\left(n, p_{2}\right): S \times{ }_{C} X \cdots \rightarrow X \times{ }_{K} X$ is dominant. Then there exists a $C$-pseudomorphism $h: S \cdots \rightarrow G^{\prime}$ such that the diagram

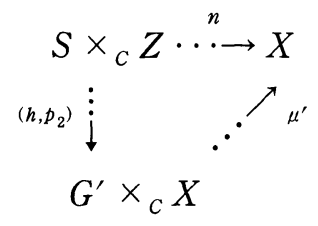

is commutative. 
Proof of Sublemma. Since we treat reduced noetherian schemes over fields of characteristic 0 , their products are reduced noetherian schemes so that the notion of pseudo-morphisms coincide with that of rational maps (E.G.A. Chap. I. §8 and Chap. IV, §20). We have a $K$-rational map $\left(\mu^{\prime}, p_{2}\right)^{-1} \circ\left(n, p_{2}\right): S \times_{C} X \cdots \rightarrow X \times_{K}$ $X \cdots \rightarrow G^{\prime} \times{ }_{C} X$ since $\left(n, p_{2}\right)$ is dominant. So by Proposition (1.11) we have a $C$-rational map $h: S \cdots \rightarrow G^{\prime}$ such that the diagram

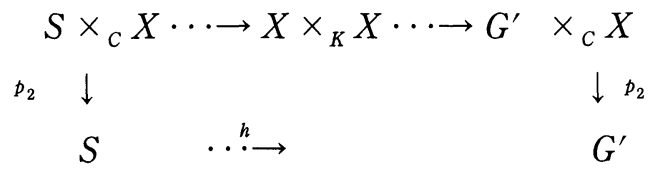

is commutative, where the upper horizontal maps are $\left(n, p_{2}\right)$ and $\left(\mu^{\prime}, p_{2}\right)^{-1}$. The assertion of Sublemma follows from the diagram.

q.e.d.

In $\mathbf{Q}\left(L \otimes_{K} L\right)$, similarly the field $L \otimes 1$ and $C_{\left.\mathbf{Q}^{(L} \otimes_{K} L\right)}$ are linearly disjoint over $C$. We have a differential isomorphism $\theta^{\prime}: \mathbf{Q}\left(L \otimes_{K} L\right) \rightarrow \mathbf{Q}\left(C_{\left.\mathbf{Q}^{(L} \otimes_{K} L\right)} \otimes_{C} L\right)$ such that $\theta^{\prime}(L \otimes 1)=1 \otimes L$. Composing $\theta^{\prime}$ with the inclusion $L \rightarrow \mathbf{Q}\left(L \otimes_{K} L\right), a \mapsto 1$ $\otimes a$ for $a \in L$, we get a differential morphism $L \rightarrow \mathbf{Q}\left(L \otimes_{K} L\right) \rightarrow \mathbf{Q}\left(C_{\mathbf{Q}\left(L \otimes_{K} L\right) \otimes_{K} L}\right.$ $L)$. This means in the language of schemes that we have a $K$-birational map $G^{\prime}$ $\times_{C} X \cdots \rightarrow X \times{ }_{K} X$ and a $K$-rational map $\mu^{\prime \prime}: G^{\prime} \times{ }_{C} X \cdots \rightarrow X$ compatible with derivation so that we have a $G^{\prime}$-rational map (Id, $\left.\mu^{\prime \prime}\right): G^{\prime} \times_{C} X \cdots \rightarrow G^{\prime} \times_{C} X$. It follows from the definition $\left(\mathrm{Id}, \mu^{\prime}\right) \circ\left(\mathrm{Id}, \mu^{\prime \prime}\right)=\left(\mathrm{Id}, \mu^{\prime \prime}\right) \circ\left(\mathrm{Id}, \mu^{\prime}\right)=\operatorname{Id}_{G^{\prime} \times_{C} X}$. In particular the rational map (Id, $\left.\mu^{\prime}\right): G^{\prime} \times{ }_{C} X \cdots \rightarrow G^{\prime} \times_{C} X$ is birational. (So by Sublemma we have a rational map $(-1): G^{\prime} \cdots \rightarrow G^{\prime}$ such that the diagram

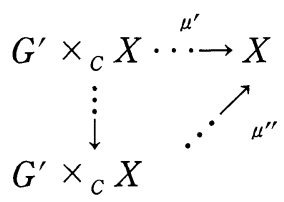

is commutative. Let us write $\mu^{\prime}(g, x)=g x$ when $\mu: G \times X \cdots \rightarrow X$ is defined at $(g, x) \in G \times{ }_{C} X$. Multiplying $\left(\mu^{\prime}, p_{2}\right): G^{\prime} \times_{C} X \cdots \rightarrow X \times_{K} X$ with $G^{\prime}$, we get a birational map

$$
G^{\prime} \times_{C}\left(G^{\prime} \times_{C} X\right) \cdots \rightarrow G^{\prime} \times_{C}\left(X \times_{K} X\right),
$$

$\left(g_{1}, g_{2}, x\right) \mapsto\left(g_{1}, g_{2} x, x\right)$. We also have a birational map induced by $\left(\mu^{\prime}, p_{2}\right)^{-1}: X$ $\times_{K} X \cdots \rightarrow G^{\prime} \times_{C} X$

$$
G^{\prime} \times_{C}\left(X \times_{K} X\right)=\left(G^{\prime} \times{ }_{C} X\right) \times_{K} X \cdots \rightarrow\left(X \times_{K} X\right) \times_{K} X
$$

$(g, x, y) \mapsto(g x, x, y)$. Composing birational maps (3.10.3), (3.10,4) with the 
projection $p_{13}: X \times{ }_{K} X \times{ }_{K} X \rightarrow X \times{ }_{K} X$, we get a dominant rational map $G^{\prime} \times{ }_{C}$ $G^{\prime} \times{ }_{c} X \cdots \rightarrow X \times X,\left(g_{1}, g_{2}, x\right) \mapsto\left(g_{1}\left(g_{2} x\right), x\right)$. Therefore we get a rational map $m^{\prime}: G^{\prime} \times G^{\prime} \cdots \rightarrow G^{\prime}$ by Sublemma (3.10.2). We show that $\left(m^{\prime}, p_{2}\right): G^{\prime} \times{ }_{c}$ $G^{\prime} \cdots \rightarrow G^{\prime} \times{ }_{C} G^{\prime}$ is dominant. We have a birational map rational map

$$
\begin{gathered}
\left(\mu^{\prime}, p_{2}\right)^{-1} \times \operatorname{Id}_{X}{ }^{\circ}\left(p_{13}, p_{2}\right): X \times_{K} X \times_{K} X \cdots \rightarrow X \times{ }_{K} X \times_{K} X \\
=\left(X \times_{K} X\right) \times_{K} X \cdots \rightarrow\left(G^{\prime} \times_{C} X\right) \times_{K} X .
\end{gathered}
$$

On the other hand, we have a birational map

$$
\begin{aligned}
G^{\prime} \times_{C} X \times_{K} X & =G^{\prime} \times_{C}\left(X \times_{K} X\right) \cdots \rightarrow G^{\prime} \times_{C}\left(G^{\prime} \times_{K} X\right) \\
& =G^{\prime} \times_{C} G^{\prime} \times_{K} X .
\end{aligned}
$$

Composing birational maps (3.10.3), (3.10.4), (3.10.5), (3.10.6), we get a birational map

$$
G^{\prime} \times{ }_{C} G^{\prime} \times{ }_{C} X \rightarrow G^{\prime} \times G^{\prime} \times_{C} X,\left(g_{1}, g_{2}, x\right) \mapsto\left(m^{\prime}\left(g_{1} g_{1}\right), g_{2}, x\right) .
$$

Hence $\left(m^{\prime}, p_{2}\right): G^{\prime} \times{ }_{c} G^{\prime} \cdots \rightarrow G^{\prime} \times{ }_{c} G^{\prime}$ is dominant. Since the rational map $\mu^{\prime}$ is defined by composite of birational automorphisms, the following diagram is commutative:

$$
\begin{aligned}
& G_{\|}^{\prime} \times G^{\prime} \times G^{\prime}=G^{\prime} \times\left(G^{\prime} \times G^{\prime}\right) \stackrel{\operatorname{Id} \times m}{\longrightarrow} G^{\prime} \times G^{\prime} \\
& \left(G^{\prime} \times G^{\prime}\right) \times G^{\prime} \\
& \vdots^{m \times I d} \\
& G^{\prime} \times G^{\prime} \\
& \stackrel{m}{\rightarrow} \quad G^{\prime}
\end{aligned}
$$

where the products are taken over $C$. In other words $m^{\prime}$ is a rational group law over $G$. Thus we can find a group scheme $G$ defined over $C$ such that $G$ is birationally equivalent to $G^{\prime}$ by Proposition (1.14). The $K$-rational map $\mu: G \times{ }_{C} X=$ $G_{K} \times_{K} X \cdots \rightarrow X$ induced of $\mu^{\prime}$ is compatible with derivations. We have seen above that the morphism $\left(p_{1}, m\right): G \times{ }_{C} X \cdots \rightarrow G \times{ }_{C} X$ is birational and hence dominant. It follows from the definition that the diagram

$$
\begin{array}{ccc}
G \times{ }_{C} G \times{ }_{C} X=G \times{ }_{C}\left(G \times{ }_{C} X\right) & \cdots \rightarrow \rightarrow & G \times{ }_{C} X \\
\| & & \vdots \\
\left(G \times{ }_{C} G\right) \times{ }_{C} X & & \downarrow^{m} \\
\vdots{ }^{\mu \times I d} & & \\
G \times{ }_{C} X & \cdots & \\
& \cdots \rightarrow & X
\end{array}
$$


is commutative so that the group scheme $G_{K}$ rationally operates on $X$. It follows from Proposition (1.16) and the argument of the proof of Theorem (2.13) that we can find a model $Z$ with derivation of $L / K$ such that $G_{K}$ operates on $Z$ and the morphism $\mu: G_{K} \times{ }_{K} Z \rightarrow Z$ is a morphism of schemes with derivation. It follows from the construction a morphism $\left(\mu, p_{2}\right): G \times{ }_{C} Z=G \times{ }_{K} Z \rightarrow Z \times Z$ is birational so that we may assume $\left(G_{K}^{\natural}, Z^{\natural}\right)$ is a principal homogeneous space. It follows from Theorem (2.13) that the extension $L / K$ is quasi-automorphic with automorphy group $G$. $L / K$ is quasi-automorphic with automorphy group $G$.

Corollary (3.11). Let $L / K$ be a quasi-automorphic extension with $C_{L}=C_{K}$ (so that $L / K$ is automorphic) and $M$ a differential intermediate field. Then the extension $L / M$ is automorphic. The automorphy group is $\mathbf{D i f f -}-\mathbf{b i r}_{M} L$ and is a closed subgroup of the automorphy group of $L / K$.

Proof. By Theorem (3.10) it is sufficient to show that the extension $L / M$ is strongly normal. Let $f, g: L \rightarrow M^{\prime}$ be two $M$-morphisms of differential fields. So in particular $f, g$ are $K$-morphisms. Since $L / K$ is strongly normal by Theorem (3.10), $f$ is strong over $g$. Thus the extension $L / M$ is strongly normal. The last assertion follows from Theorem (2.21).

Remark (3.12). For general quasi-automorphic extension $L / K$, the extension $L / K$ is not always quasi-automorphic for a differential intermediate field $M$ of $L / K$. See Remark (4.2) and Example (4.9).

\section{§4. Galois correspondence}

Let $L / K$ be a quasi-automorphic extension with automorphy group $G$ so that $G$ is a $C_{K}$-algebraic group scheme. Let $X$ be a model with derivation of $L / K$ such that $G$ operates on $X$ and $\left(\mu, p_{2}\right): G \times{ }_{C_{K}} X \rightarrow X \times_{K} X$ is an isomorphism of schemes with derivation (cf. Theorem (2.13)). Let $H$ be a $C_{K}$-algebraic subgroup scheme. We say that an element $a \in L$ remains invariant under the operation of $H$ or $a$ is $H$-invariant if the following condition is satisfied: For any $K^{k}$-scheme $S$ and for any $g \in H(S)$ so that $g$ induces an $S \otimes_{C_{K}} K$-automorphism $\phi_{g}: S \times_{C_{K}} X$ $\rightarrow S \times{c_{K}}_{K} X$, the meromorphic function $a^{\circ} p_{2}$ coincides with $a^{\circ} p_{2}{ }^{\circ} \psi_{g}$. The meromorphic function $a$ is $H$-invariant if and only if $a^{\circ} p_{2}^{\circ} \mu_{H}=a^{\circ} p_{2}$, where $\mu_{H}: H \times_{C_{K}} X$ $\rightarrow X$ is the morphism of operation of $H$ on $X$. For a $C_{K}$-algebraic subgroup scheme $H$, we denote by $L^{H}$ the differential intermediate field of $L / K$ consisting of elements of $L$ invariant under $H$. Let now $M$ be an intermediate differential field 
of $L / K$. We denote by $\mathscr{G}(M):\left(\mathrm{Sch} / C_{K}\right) \rightarrow(\mathrm{Grp})$ the subgroup functor of $G$ leaving every element of $M$ fixed.

Lemma (4.1). Let $L / K$ be a quasi-automorphic extension with automorphy group $G$. Then the group functor $G(M)$ is representable by a closed $C_{K^{-}}$algebraic subgroup scheme of $G$.

Proof. We denote $C_{K}$ by $C$. Let $Y$ be a model with derivation of $M / K$ so that we have a $K$-rational map $\varphi: X \cdots \rightarrow Y$ induced by the inclusion map $M \subset L$. We have to show that for a smooth $C$-scheme $S$ and an $S \otimes_{C} K$-pseudo-morphism $\phi: S \times{ }_{C} X \cdot \rightarrow S \times{ }_{C} X$ compatible with derivation, there exists a closed $C$-subscheme $S_{0}$ of $S$ such that for any $C$-morphism $f: S^{\prime} \rightarrow S$ of schemes, the diagram

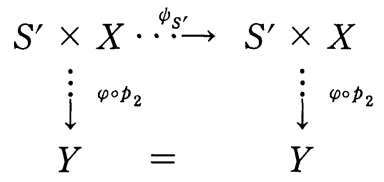

is commutative if and only if the morphism $f: S^{\prime} \rightarrow S$ factors through the subscheme $S_{0}$, where the fibre product is taken over $C$. Since the question is local, we may assume that $S$ and $X$ are affine: $X=\operatorname{Spec} A$ with a $C$-algebra $A$. Let $g$ be a point of $S$. Then $\phi_{g}: X_{C(g) \otimes_{C} K} \cdots \rightarrow X_{C(g) \otimes_{C} K}$ factors through $\varphi^{\circ} p_{2}$ if and only if we have

$$
\left(u^{\circ} \varphi^{\circ} p_{2}\right) \circ \phi_{g}=u^{\circ} \varphi^{\circ} p_{2}
$$

for every $u \in M$, where we regard $u$ as a meromorphic function on $Y$. The relations (4.1.1) are reduced to relations with coefficients in $K$ among the coordinates of the point $g$ of the affine scheme $S$ so that $\phi_{g}: X_{C(g) \otimes_{C} K} \cdots \rightarrow X_{C(g) \otimes_{C} K}$ factors through $\varphi^{\circ} p_{2}$ if and only if the coordinates of the point $g$ of the affine scheme $S$ satisfies a certain number of polynomial relations

$$
F_{\alpha}(g)=0, \quad \alpha \in I
$$

with coefficients in $K$, which is an equality in $C(g) \bigotimes_{C} K$. Since $C(g)$ and $K$ are linearly disjoint over $C$ in $C(G) \otimes_{C} K$, there exist a certain number of polynomial relations

$$
H_{\beta}(g)=0, \quad \beta \in J
$$

with coefficients in $C_{K}$ such that $g$ satisfies (4.1.2) if and only if $g$ satisfies 
Remark (4.2). Let $L / K$ be a quasi-automorphic extension with automorphy group $G$ and $M$ a differential intermediate field. In general the algebraic group $\mathscr{G}(M)$ is small so that $L / M$ is not quasi-automorphic with respect to $\mathscr{G}(M)$. Let us illustrate the situation by an example. We work in the field $\overline{\mathbf{Q}}[[x]]\left[x^{-1}\right]$ of Laurent series with derivation $d / d x$. Let $K=\mathbf{Q}$ and $y=\exp x$. We set $L=$ $\mathbf{Q}(\omega, y)$, where $\omega$ is a complex number satisfying $\omega^{2}+\omega+1=0$ so that $\omega^{3}=$ 1. We have $C_{L}=\mathbf{Q}(\omega) \neq \mathbf{Q}=C_{K}$. Since the extension $C_{L} / C_{K}$ is Galois with group $\mathbf{Z} / 2 \mathbf{Z}$ and since $\mathbf{Q}(\omega)\left[y, y^{-1}\right]=\mathbf{Q}(\omega) \bigotimes_{\mathbf{Q}} \mathbf{Q}\left[y, y^{-1}\right]$, Spec $\mathbf{Q}(\omega)\left[y, y^{-1}\right]$ is a principal homogeneous space of $\mathbf{G}_{m} \mathbf{Q} \times \mathbf{Z} / 2 \mathbf{Z}$. (See also Example (3.2).) To be precise $(g, 0),(g, \overline{1}) \in \mathbf{G}_{m} \mathbf{Q} \times \mathbf{Z} / 2 \mathbf{Z}$ operates on $\mathbf{Q}(\omega)\left[y, y^{-1}\right]$ over $\mathbf{Q}$ by $(g, 0)(\omega)=\omega,(g, 0)(y)=g y(=g \otimes y),(g, \overline{1})(\omega)=\omega^{2},(g, \overline{1})(y)=g \otimes y$. So by Theorem (2.13), the extension $L / K$ is quasi-automorphic. Let now $M=$ $\mathbf{Q}\left(\omega y^{3}\right)$ so that $\left(\omega y^{3}\right)^{\prime}=3 \omega y^{3}$ and $M$ is a differential intermediate field of $L / K$. Let $(g, \overline{0}) \in \mathbf{G}_{m \mathbf{Q}} \times \mathbf{Z} / 2 \mathbf{Z}$. Then $(g, \overline{0})$ is in $\mathscr{G}(M)$ if and only if $(g, \tilde{0})$ fixes $\omega y^{3}: \omega y^{3}=g^{3} \otimes \omega y^{3}$ or equivalently $g^{3}=1$. Let us look for a condition for an element $(g, \overline{1})$ to be in $\mathscr{G}(M)$. Since $(g, \overline{1})\left(\omega y^{3}\right)=g^{3} \otimes \omega^{2} y^{3}$ so that $(g, \overline{1})$ is in $\mathscr{G}(M)$ if and only if $g^{3} \otimes \omega^{2} y^{3}=\omega y^{3}=1 \otimes \omega y^{3}$. So $(g, \overline{1})$ is never in $\mathscr{G}(M)$. Hence we have $\mathscr{G}(M)=\left\{(g, \overline{0}) \in \mathbf{G}_{m \mathbf{Q}} \times \mathbf{Z} / 2 \mathbf{Z} \mid g^{3}=1\right\}$. Since $L / M$ is algebraic of degree 6 and $\mathscr{G}(M)$ is a finite group scheme of degree $3, \mathscr{G}(M)$ can not be an automorphy group of $L / M$. We notice here that the extension $L / M$ is quasi-automorphic with automorphy group $\mathscr{G}(M) \times \mathbf{Z} / 2 \mathbf{Z}$. See example (4.4).

THEOREM (4.3) (Kolchin). Let $L / K$ be a strongly normal extension or according to Theorem (3.10) equivalently a quasi-automorphic extension with $C_{L}=C_{K}$. Then there exists an order reversing 1:1 correspondence between the elements of the following two sets.

(i) The set of differential intermediate fields between $L$ and $K$.

(ii) The set the $C_{K}$-algebraic subgroup schemes of the automorphy group $G$ (which is a $C_{K^{-}}$algebraic group scheme).

To a differential intermediate field $M$, we associate the closed $C_{K^{-}}$algebraic subgroup scheme $\mathscr{G}(M)$. A $C_{K^{-}}$algebraic subgroup scheme $H$ of $G$ is mapped to the differential intermediate field $L^{H}$ of $H$-invariants.

Proof. Since $L / K$ is strongly normal, it follows from Corollary (3.11) that for a differential intermediate field $M, L / M$ is quasi-automorphic with automorphy group $\operatorname{Diff}_{-} \mathbf{b} \mathbf{i r}_{M} L$ and $\mathscr{G}(M)=\mathbf{D i f f}-\mathbf{b} \mathbf{i r}_{M} L$. Hence $L^{\mathscr{G}(M)}=M$ by Lemma 
(2.22). We have to show that for any closed $C_{K}$-algebraic subgroup scheme $H$, $\mathscr{G}\left(L^{H}\right)=H$. The inclusion $\mathscr{G}\left(L^{H}\right) \supset H$ is evident from the definition. Let us denote $\mathscr{G}\left(L^{H}\right)$ by $H_{1}$ which is a $C_{K^{-}}$algebraic subgroup scheme of $G$ by Lemma (4.1) so that we have

$$
L^{H_{1}}=L^{H}
$$

as the above argument shows. Geometrically let $X$ be a model with derivation of $L / K$ such that $G$ operates on $X$ as in Theorem (2.13). If we denote by $\left(H_{K} \backslash X\right)^{\prime}$ and $\left(H_{1 K} \backslash X\right)^{\prime}$, the varieties of orbits of $H_{K}$ and $H_{1 K}$ respectively, then the natural morphism $\left(H_{K} \backslash X\right)^{\prime} \rightarrow\left(H_{1 K} \backslash X\right)^{\prime}$ is birational. Let us consider a base change $X_{L}$ of $X$ by the field extension $L / K$. Since we have the generic point Spec $L \rightarrow X, X_{L}$ is isomorphic to $G_{L}=G \otimes_{C_{K}} L$. It follows from (4.3.1) the canonical morphism $H_{L} \backslash G_{L} \rightarrow H_{1 L} \backslash G_{L}$ is birational so that we have $H_{L}=H_{1 L}$ and consequently $H=H_{1}$.

We introduced the notion of quasi-automorphic extension and showed that classical Galois extension and strongly normal extension are quasi-automorphic. Further for these two types of extensions the automorphy group is uniquely determined and we have the Galois correspondence (Theorems (2.21) and (4.3)). In genbral a quasi-automorphic extension is not so nice as these two types of extensions. In fact we showed already in Remark (4.2) that in general we do not have the Galois correspondence since in that example the subgroup functor $\mathscr{G}(M)$ of $G$ is too small. Besides, the automorphy group is not uniquely determined as the following example shows. Also Example (4.9) offers us such an example, where $L / K$ is an abstract field extension.

Example (4.4). We work in the field $\overline{\mathbf{Q}}[[x]][x]$ of Laurent series with derivation $d / d x$. Let us set $K=\mathbf{Q}, y=\exp x, L=\mathbf{Q}(\sqrt{-1}, y)$. Then as in Remark (4.2), $\operatorname{Spec} \mathbf{Q}(\sqrt{-1})\left[y, y^{-1}\right]$ is a principal homogeneous space with a group $\mathbf{G}_{m \mathbf{Q}} \times \mathbf{Z} / 2 \mathbf{Z}$. So $L / K$ is quasi-automorphic with automorphy group $\mathbf{G}_{m} \mathbf{Q}$ $\times \mathbf{Z} / 2 \mathbf{Z}$ by Theorem (2.13). Another principal homogeneous space structure is defined as follows. We consider an algebraic group $G$ generated by the following $G_{\mathbf{Q}^{-}}$automorphisms of $L \otimes_{C_{K}} G$ :

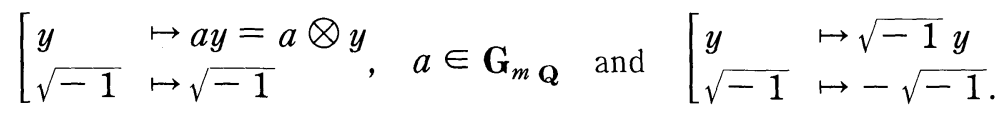

Namely as a $\mathbf{Q}$-scheme $G$ is a disjoint union of two copies of $\mathbf{A}^{*}=\mathbf{A}_{\mathbf{Q}}^{1}-(0)$ : $G=G_{0} \amalg G_{1}$ with $G_{0}, G_{1} \simeq \mathbf{A}^{*}$. So we have on $\mathbf{A}^{*}$ the usual product. $A$ point 
$a \in G_{0}$ represents a $G_{\mathbf{Q}^{-}}$automorphism

$$
\left[\begin{array}{ll}
y & \mapsto a y=a \otimes y \\
\sqrt{-1} & \mapsto \sqrt{-1}
\end{array}\right.
$$

and a point $a \in G_{1}$ represents a $G_{\mathbf{Q}}$-automorphism

$$
\left[\begin{array}{ll}
y & \mapsto a y=a \otimes \sqrt{-1} y \\
\sqrt{-1} & \mapsto-\sqrt{-1}
\end{array}\right.
$$

of $L \otimes_{C_{K}} G$. The group law on $G$ is described as follows: If $a_{1}, a_{2} \in \mathbf{A}^{*}=G_{0}$, then the product of $a_{1}$ and $a_{2}$ is the usual product $a_{1} a_{2} \in \mathbf{A}^{*}=G_{0}$; If $a \in \mathbf{A}^{*}$ $=G_{0}, b \in \mathbf{A}^{*}=G_{1}$, then the product of $a$ and $b$ is $a b \in \mathbf{A}^{*}=G_{1} ;$ If $b_{1}, b_{2} \in$ $\mathbf{A}^{*}=G_{1}$, then the product of $b_{1}$ and $b_{2}$ is $-b_{1} b_{2} \in \mathbf{A}^{*}=G_{0}$. So we have an exact sequence

$$
1 \rightarrow \mathbf{G}_{m \mathbf{Q}} \rightarrow G \rightarrow \mathbf{Z} / 2 \mathbf{Z} \rightarrow 1
$$

The extension (4.4.1) does not split over $\mathbf{Q}$. However if we go to $\mathbf{Q}(\sqrt{-1})$, then the extension splits so that $\left(G, \operatorname{Spec} \mathbf{Q}(\sqrt{-1})\left[y, y^{-1}\right] / \mathbf{Q}\right.$ is a principal homogeneous space. Hence $L / K$ is quasi-automorphic with automorphy group $G$ by Theorem (2.13).

We considering Remark (4.2), what we can expect best in general is the following

Proposition (4.5). Let $L / K$ be a quasi-automorphic extension. Then the mapping $\{C$-algebraic subgroups schemes of $G\} \rightarrow\{$ differential intermediate fields of $L / K\}$, $L \mapsto L^{H}$ is injective.

Proof. Let $H_{1}, H_{2}$ be two algebraic subgroups of $G$ such that $L^{H_{1}}=L^{H_{2}}$. We have to show $H_{1}=H_{2}$. Considering the subgroup scheme of $G$ generated by $H_{1}$ and $H_{2}$, we may assume that $H_{2}$ is an algebraic subgroup of $H_{1}$. Proposition now follows from the latter part of the proof of Theorem (4.3).

Proposition (4.5) does not seem interesting nor useful since the conclusion of the proposition holds simply if $L / K$ is an abstract field extension such that there exists a model $X$ of $L / K$ which is a principal homogeneous space for an algebraic group $G$ over $K$. When $L / K$ is an abstract field extension of char. $p>0$, Proposition (4.5) is proved in [Ch] (Theorem 7.6 of $[\mathrm{Ch}]$ ) in order to study inseparable field extension. See also [G]. For an automorphic extension we have a map from in- 
termediate fields to subgroups (cf. Theorem (4.10)).

Let $L / K$ be an abstract field extension which is quasi-automorphic with automorphy group $G$. In particular the extension $L / K$ is finite: We set $n=$ [L:K]. Let $M / K$ be a Galois extension containing $L$ and $\mathscr{G}$ its Galois group. We can take $M=$ the Galois closure of $L / K$ as they did in [G]. However it is not necessary to assume that $M$ is the Galois closure of $L / K$. Since $M \otimes_{K} M$ is a direct product of fields isomorphic to $M, L \bigotimes_{K} M$ is also a direct product of fields isomorphic to $M: L \bigotimes_{K} M \simeq \Pi_{i=1}^{n} M_{i}$ with $M_{i} \simeq M$. Geometrically the base change Spec $L \otimes_{K} M$ of Spec $L$ over $K$ is a disjoint union of $n$-copies of Spec $M$. So $G \otimes_{K} M$ is a subgroup scheme of $\operatorname{Aut}_{M}\left(\operatorname{Spec} L \bigotimes_{K} M\right)$ which is isomorphic to the symmetric group $S_{n}$ of degree $n$. Hence $\left(G \otimes_{K} L\right.$, Spec $\left.L \bigotimes_{K} M\right)$ is $M$ isomorphic to an operation of a finite group $H$ on the set $S=\operatorname{Spec} L \bigotimes_{K} M \simeq$ Spec $\Pi_{i=1}^{n} M_{i}$ of $n$-points such that $(H, S)$ is a principal homogeneous space. On the other hand the Galois group $\mathscr{G}$ of $M / K$ operates on $\left(G_{M}\right.$, $\left.\operatorname{Spec} L \bigotimes_{K} M\right)$ as $K$-automorphisms covering the operation of the Galois group $\mathscr{G}$ on $M$ giving a descent data on $\left(H\right.$, Spec $\left.\Pi_{i=1}^{n} M_{\imath}\right)$. In particular $\mathscr{G}$ operates on the set $S$ such that $\mathscr{G}$ normalizes the subgrup $H$ of $S_{n}$.

Remark (4.6). Let us consider in general an $M$-scheme Spec $\Pi_{i=1}^{l} M_{i}, M_{i}=$ $M$, where we regard $\Pi_{t=1}^{l} M_{i}$ as an $M$-algebra by the diagonal map. To give a Galois descent on the $M$-scheme $\Pi_{i=1}^{l} M_{i}$ is equivalent to defining a morphism $\rho$ : $\mathscr{G} \rightarrow S_{l}, S_{l}$ being the symmetric group of degree $l$. In fact a morphism $\rho: \mathscr{G} \rightarrow S_{l}$ defines an operation of the Galois group $\mathscr{G}$ on the algebra $\Pi_{i=1}^{l} M_{i}$ by sending $\left(x_{1}\right.$, $\left.x_{2}, \ldots, x_{l}\right) \in \Pi_{i=1}^{l} M_{i}$ to $\left(\sigma\left(x_{(\rho \sigma)(1)}\right), \sigma\left(x_{(\rho \sigma)(2)}\right), \ldots, \sigma\left(x_{(\sigma o)(l)}\right)\right)$ for $\sigma \in \mathscr{G}$. The operation covers that of $\mathscr{G}$ on $M$. Namely the structure morphism Spec $\Pi_{i=1}^{l} M_{\imath} \rightarrow$

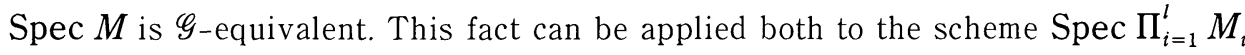
and the group scheme $H$.

According to $[G]$ we adopt the following

Definition (4.7). Let $H$ be a finite group operating effectively on a set $S$ of $n$ letters so that $H$ is a subgroup of the symmetric group $S_{n}$. We say that $H$ is regular if $(H, S)$ is a principal homogeneous space.

The following theorem is due to Greither and Pareigis ([G] 2.1 Theorem). 
THEOREM (4.8). Keeping the notation above, we have a 1:1-correspondence between the elements of the following sets:

(1) The set of equivalence classes of finite group schemes $G$ operating on $\operatorname{Spec} L$ over $K$ such that $L / K$ is quasi-automorphic with automorphy group $G$ (Here we say that two operations $\left(G_{1}, \operatorname{Spec} L\right)$ and $\left(G_{1}\right.$, Spec $\left.L\right)$ are equivalent if there exists a morphism $\varphi: G_{1} \rightarrow G_{2}$ of $K$-group schemes such that $(\varphi, \mathrm{Id}):\left(G_{1}\right.$, Spec $\left.L\right) \rightarrow\left(G_{2}\right.$, $\operatorname{Spec} L$ ) is an isomorphism of group scheme operations.);

(2) The set of equivalence classes of finite groups $H$ operating effectively on the set $S$ of $n$-letters (so that $H$ can be regarded as a subgroup of the symmetric group $S_{n}$ of degree $n$ ) such that $H$ is regular and normalized by (the image in $S_{n}$ of) the Galois Group $\mathscr{G}$ (We say that two finite groups operating on the set $S$ are equivalent if their images in the symmetric group $S_{n}$ coincide, the operation of the Galois group $\mathscr{G}$ on the set $S$ being fixed.).

Proof. It would be enough to understand how we define the correspondence. From data in (1) we define $\left(G \otimes_{K} M\right.$, Spec $\left.L \otimes_{K} M\right)$ which is $M$-isomorphic to the operation $(H, S)$ of a finite group $H$ on the set $S$, as we have seen above. Conversely given a data in (b) or let $(H, S)=\left(H, \operatorname{Spec} \bigotimes_{K} M\right)$ be a principal homogeneous space (over $M$ ). The operation of the Galois group $\mathscr{G}$ defines a descent data on $\left(H, \operatorname{Spec} L \bigotimes_{K} M\right)$ by Remark (4.6). So $\left(H^{\mathscr{G}},\left(\operatorname{Spec} L \bigotimes_{K} M\right)^{\mathscr{G}}\right)=$ $\left(H^{\mathscr{G}}, \mathrm{Spec} L\right)$ is a principal homogeneous space (over $K$ ) by the Galois descent. Here $H^{\mathscr{G}}$ denotes the descent of the $M$-scheme $H$ by the descent data. Namely let $A$ be an $M$-Hopf algebra such that $\mathscr{G}$ operates on $A$ and $H^{\mathscr{G}}=\operatorname{Spec} A^{\mathscr{G}}$, where $A^{\mathscr{G}}$

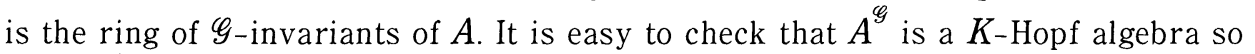
that $H^{\mathscr{G}}$ is a finite group scheme and $\left(H^{\mathscr{G}}, \operatorname{Spec} L\right)$ is a principal homogeneous space.

Therem (4.8) yields us examples of quasi-automorphic extensions of abstract fields such that the automorphy group is not uniquely determined.

Example (4.9) ([G], 2.3). We take $K=\mathbf{Q}, L=\mathbf{Q}(\sqrt[4]{2})$ and $M=\mathbf{Q}(\sqrt[4]{2}$, $\sqrt{-1}$ ). So the Galois group $\mathscr{G}$ is isomorphic to the dihedral group: Letting $\operatorname{Spec} L$ $\otimes_{K} M=\{1,2,3,4\}$, we can identify $\mathscr{G}$ with a subgroup $\langle\sigma, \tau\rangle, \sigma=(1234), \tau=$ (13). A subgroup $H=\langle\sigma\rangle$ of the symmetric group $S_{4}$ is a regular subgroup and normalized by the Galois group $\mathscr{G}$. On the other hand $\left\langle\sigma^{2}, \sigma \tau\right\rangle$ is also a regular subgroup and normalized by $\mathscr{G}$. Since $H \simeq \mathbf{Z} / 4 \mathbf{Z},\left\langle\sigma^{2}, \sigma \tau\right\rangle \simeq \mathbf{Z} / 2 \mathbf{Z}+\mathbf{Z} / 2 \mathbf{Z}$, these two data in (ii) of Theorem (4.8) define non-isomorphic automorphy groups for $\mathbf{Q}(\sqrt[4]{2}) / \mathbf{Q}$. 
Proposition (4.5) gives an injective map from the set of algebraic subgroups of $G$ to the set of differential intermediate fields of $L / K$. If an extension is automorphic, we have the inverse of the above map as the following theorem shows.

THEOREm (4.10). Let $L / K$ be an automorphic extension. Then there exists an order reversing 1:1 correspondence between the elements of the following two sets.

(i) The set of differential fields between $L$ and $K$.

(ii) The set of $C_{K}$-algebraic subgroup schemes of the automorphy group $G$ (which is a $C_{K}$-algebraic group scheme).

To a differential intermediate field $M$ we associate the closed $C_{K}$-algebraic subgroup scheme $\mathscr{G}(M)$. A $C_{K^{-}}$algebraic subgroup scheme $H$ of $G$ is mapped to the differential intermediate field $L^{H}$ of $H$-invariants.

Proof. The argument of the proof of Theorem (4.3) allows us to prove that $\mathscr{G}\left(L^{H}\right)=H$ for any closed $C_{K}$-subgroup scheme of $G$. So it is sufficient to show $L^{\mathscr{G}(M)}=M$ for every differential intermediate field $M$. The inclusion $L^{\mathscr{G}(M)} \supset M$ being trivial, we have to check the other inclusion $L^{\mathscr{G}(M)} \subset M$. We shall show that if $y \in L \backslash M$, then $y \notin L^{\mathscr{G}(M)}:$ If $y \in L \backslash M$, then there exist $A \in \operatorname{ab}\left(\mathrm{Fld} / K^{\natural}\right)$ and $h \in \mathscr{G}(M)(A)$ such that $\phi_{h}^{*}(1 \otimes y) \neq 1 \otimes y$. We need a

Sublemma (4.11). If $y \in L \backslash M$, then there exist an $A \in$ ob (Fld $\left./ M^{\natural}\right)$ and $M$-homomorphisms $\lambda_{1}, \lambda_{2}: L^{\natural} \rightarrow A$ such that $\lambda_{1}(y) \neq \lambda_{2}(y)$.

The Sublemma follows from the proof of Lemma (3.5).

The morphisms $\lambda_{1}, \lambda_{2}: L^{\natural} \rightarrow A$ define differential morphisms $l_{1}, l_{2}: L \rightarrow$ $A[[t]]\left[t^{-1}\right]$ by Proposition (1.4) so that $l_{1}, l_{2} \in \mathscr{F}_{L / M}(A) \subset \mathscr{F}_{L / K}(A)$. Let $X$ be a model of the differential field extension $L / K$ on which the automorphy group $G$ operates. Since $L / K$ is quasi-automorphic, we can find $h \in G(A)$ such that $l_{2}=$ $h l_{1}$. Let $\varphi_{1}, \varphi_{2}: \operatorname{Spec} A[[t]]\left[t^{-1}\right] \rightarrow \operatorname{Spec} L \rightarrow X$ be the morphisms of schemes with derivation corresponding to $l_{1}, l_{2}$ respectively (cf. $\S 2$ ). We denote the morphisms of the base change $\operatorname{Spec} A[[t]]\left[t^{-1}\right] \rightarrow A \otimes_{C_{K}} X$ of $\varphi_{1}, \varphi_{2}$ by $\varphi_{1 A}, \varphi_{2 A}$ so that we have commutative diagrams

$$
\begin{array}{ccc}
\operatorname{Spec} A[[t]]\left[t^{-1}\right] \stackrel{\varphi_{i A}}{\longrightarrow} A \otimes_{C_{K}} X \\
& \begin{array}{c}
\downarrow p_{2} \\
\varphi_{i} \searrow
\end{array} & X
\end{array}
$$

Let $\phi_{h}: A \otimes_{C_{K}} X \rightarrow A \otimes_{C_{K}} X$ be the morphism induced by the operation of the 
$A$-valued point $h$. So we have $\varphi_{2 A}=\phi_{h}{ }^{\circ} \varphi_{1 A}$. Since we have $\lambda_{1}(y) \neq \lambda_{2}(y)$, we have $l_{1}(y) \neq l_{2}(y)$. Since $\varphi_{1}^{*}$ and $\varphi_{2}^{*}$ coincides with respectively $l_{1}, l_{2}$ on $L, \varphi_{1 A}^{*}(1$ $\left.\otimes y-\phi_{h}^{*}(1 \otimes y)\right)=\varphi_{1 A}^{*}(1 \otimes y)-\varphi_{2 A}^{*}(1 \otimes y)=1 \otimes l_{1}(y)-1 \otimes l_{2}(y) \neq 0$ and $1 \otimes y-\phi_{h}^{*}(1 \otimes y) \neq 0$. It remains to show that $h \in \mathscr{G}(M)$ or $\phi_{h}^{*}(1 \otimes z)=$ $1 \otimes z$ for every $z \in M$. Let now $z \in M$, then

$$
\varphi_{1}^{*}(z)=\varphi_{2}^{*}(z)
$$

since $\lambda_{1}, \lambda_{2}$ are $M$-morphisms. It follows from (4.12) and (4.13), $\varphi_{1 A}^{*}(1 \otimes z)=$ $\varphi_{2 A}^{*}(1 \otimes z)$ and hence

$$
\varphi_{1}^{*}\left(1 \otimes z-\phi_{h}^{*}(1 \otimes z)\right)=0 .
$$

Since $L / K$ is automorphic, it follows from (4.14) $1 \otimes z-\phi_{h}^{*}(1 \otimes z)=0$. This is what we had to show.

We have seen in Examples (4.4) and (4.9) that for a quasi-automorphic extension, the automorphy group is not uniquely determined and we do not have the Galois correspondence. As for an automorphic extension, we have the Galois correspondence (Theorem (4.10)) but as we see below, the automorphy group is not uniquely determined. Example (4.9) yields such an example.

Example (4.15). Let $L=\mathbf{Q}(\sqrt[4]{2}), K=\mathbf{Q}$ as in Example (4.9). We have seen there that the extension $L / K$ is quasi-automorphic for mutually non-isomorphic automorphic group schemes. We show that $L / K$ is almost classically Galois and hence automorphic by Corollary (2.26) with respect to these group schemes. To this let us use a different interpretation. The Galois closure of $L$ over $K$ is $\mathbf{Q}(\sqrt[4]{2}, \sqrt{-1})$. We have a $\mathbf{Q}(\sqrt{-1})$-automorphism $\sigma$ of $\mathbf{Q}(\sqrt[4]{2}, \sqrt{-1})$ sending $\sqrt[4]{2}$ to $\sqrt[4]{2} \cdot \sqrt{-1}$ so that the Galois group of $\mathbf{Q}(\sqrt[4]{2}, \sqrt{-1}) / \mathbf{Q}(\sqrt{-1})$ is the cyclic group $\langle\sigma\rangle$ of order 4 . On the other hand we have a $\mathbf{Q}(\sqrt[4]{2})$-automorphism $\tau$ of $\mathbf{Q}(\sqrt[4]{2}, \sqrt{-1})$ sending $\sqrt{-1}$ to $-\sqrt{-1}$. The Galois group of $\mathbf{Q}(\sqrt[4]{2}, \sqrt{-1}) /$ $\mathbf{Q}(\sqrt[4]{2})$ is the cyclic group $\langle\tau\rangle$ of order 2. We have $\tau \sigma=\sigma^{3} \tau$ and the Galois group of $\mathbf{Q}(\sqrt[4]{2}, \sqrt{-1}) / \mathbf{Q}$ is the dehedral group $\langle\sigma, \tau\rangle$. In the diagram

$$
\begin{aligned}
& \mathbf{Q}(\sqrt[4]{2})-\quad \begin{array}{l}
\mathbf{Q}(\sqrt[4]{2}, \sqrt{-1}) \\
\mid \quad
\end{array} \quad \text { Galois with group }\langle\sigma\rangle \\
& \mathbf{Q}(\sqrt{-1})
\end{aligned}
$$

subfields $\mathbf{Q}(\sqrt[4]{2})$ and $\mathbf{Q}(\sqrt{-1})$ are linearly disjoint over $\mathbf{Q}$ so that $\mathbf{Q}(\sqrt[4]{2}$, $\sqrt{-1})$ is isomorphic to $\mathbf{Q}(\sqrt[4]{2}) \bigotimes_{\mathbf{Q}} \mathbf{Q}(\sqrt{-1})$. The Galois group $\langle\tau\rangle$ of $\mathbf{Q}(\sqrt{-1})$ / 
$\mathbf{Q}$ normalizes the Galois group $\langle\sigma\rangle$ of $\mathbf{Q}(\sqrt[4]{2}, \sqrt{-1}) / \mathbf{Q}(\sqrt{-1})$. So by Galois descent the operation of the finite group $\langle\sigma\rangle$ on $\mathbf{Q}(\sqrt[4]{2}, \sqrt{-1}) / \mathbf{Q}(\sqrt{-1})$ descends to an action of a finite group scheme which is a $\mathbf{Q}$-form of the cyclic group $\langle\sigma\rangle$, on $\mathbf{Q}(\sqrt[4]{2}) / \mathbf{Q}$. This operation is nothing but the quasi-automorphic structure induced by the regular subgroup $H=\langle\sigma\rangle$ in Example (4.9) so that this quasi-automorphic structure is almost classically Galois.

Now we consider the subgroup $\left\langle\sigma^{2}, \sigma \tau\right\rangle$ of the Galois group $\langle\sigma, \tau\rangle$ of $\mathbf{Q}(\sqrt[4]{2}$, $\sqrt{-1}) / \mathbf{Q}$. The corresponding subfield $\mathbf{Q}(\sqrt[4]{2}, \sqrt{-1})^{\left\langle\sigma^{2}, \tau\right\rangle}=\mathbf{Q}(\sqrt{-2})$. In the diagram

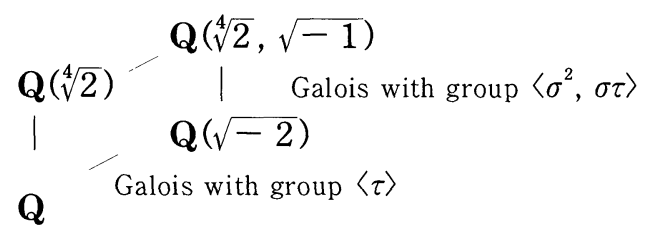

subfields $\mathbf{Q}(\sqrt[4]{2})$ and $\mathbf{Q}(\sqrt{-2})$ are linearly disjoint over $\mathbf{Q}$. Since the Galois group $\langle\tau\rangle$ of $\mathbf{Q}(\sqrt{-2}) / \mathbf{Q}$ normalizes the subgroup $\left\langle\sigma^{2}, \sigma \tau\right\rangle$. The operation of the Galois group $\left\langle\sigma^{2}, \sigma \tau\right\rangle$ on $\mathbf{Q}(\sqrt[4]{2}, \sqrt{-1}) / \mathbf{Q}(\sqrt{-2})$ descends to a finite group scheme action on $\mathbf{Q}(\sqrt[4]{2}) / \mathbf{Q}$ by Galois descent. This operation is the second quasi-automorphic structure on $\mathbf{Q}(\sqrt[4]{2}) / \mathbf{Q}$. So this structure is also almost classically Galois. We have thus seen that $\mathbf{Q}(\sqrt[4]{2}) / \mathbf{Q}$ has two mutually non-equivalent automorphic structures.

\section{REFERENCES}

[B] Bialinicki-Birula A., On Galois theory of fields with operators, Amer. J. Math., 84 (1962), 89-109.

[Bo] Bourbaki, N., Groupes et algèbres de Lie, Chapitres 2 et 3, Hermann, Paris, 1972.

[Bu] Buium, A., Differential algebraic groups of finite dimension, L. N. in Math., 1506, Springer Verlag, Berlin-Heidelberg-New York, 1992.

[C] Cassidy, F., Differential algebraic groups, Amer. J. Math., 94 (1972), $891-954$.

[Ch] Chase, S.U. and Sweedler, M., Hopf algebra and Galois theory, L. N. in Math., 97, Springer-Verlag, Berlin-Heidelberg-New York, 1969.

[D] Demazure, M., Sous-groupes algébriques de rang maximum du groupe de Cremona, Ann. Sci. Ecole Normale Sup. 4e séries, 3 (1970), 507-588.

[Dr] Drach, J., Essai sur une théorie générale de l'intégration et sur la classification des transcendentes, Ann. Sci. Ecole Normale Sup., (3) 15 (1898), 243-384.

[E.G.A] Grothendieck, A. et Dieudonné, J., Elément de Géometrie Algébrique I-IV, Grundl. Math. Wiss. Bd166, Springer-Verlag, Berlin-Heidelberg-New York, 1970 et Pub. Math. I.H.E.S., 8 (1961), 11 (1961), 17 (1963), 20 (1964), 24 
(1965), 28 (1966), 32 (1967).

[G] Greither, C. and Pareigis, B., Hopf Galois theory for separable field extensions, J. Algebra, 106 (1987), 239-258.

[K1] Kolchin, E., Differential algebra and algebraic groups, Academic Press, New York and London, 1973.

[K2] - Differential algebraic groups, Academic Press, New York and London, 1984.

[L] Lang, S., Algebra, Addison-Wesley, Reading Massachussets, 1965.

[O] Okugawa, K., Basic property of differential fields of an arbitrary characteristic and the Picard-Vessiot theory, J. Math, Kyoto Univ., 2-3 (1963), $295-322$.

[P] Picard, E., Sur les équations différentielles et les groupes algébriques de transformation, Ann. Fac. Sci. Univ. Toulouse, 1 (1887), 1-15.

[Po] Pommaret, J.F., Lie pseudogroups and mechanics, Gordon and Breach, London 1987.

[R] Rosenlicht, M., Some basic theorems on algebraic groups, Amer. J. Math., 77 (1955), 355-391.

[S.G.A.3] Demazure, M. et Grothendieck, A., Schemas en groupes I, II, III, L. N. in Math., 151, 152 153, Springer-Verlag, Berlin-Heidelberg-New York, 1971.

[U1] Umemura, H., Sur les sous-groupes algébriques primitifs du groupes de Cremona à trois variables, Nagoya Math. J., 79 (1980), 49-67.

[U2] - Algebro-geometric problems arising from Painlevé's works, Algebraic and Topological theories - to the memory of Dr. Takehiko MIYATA (1985), 467-495, Kinokuniya, Tokyo.

[U3] - Differential Galois theory of infinite dimension, Nagoya Math. J., 144 (1996), 59-135.

[W] Weil, A., On algebraic groups of transformations, Amer. J. Math., 77 (1955), $355-391$.

Graduate School of Polymathematics

Nagoya University

Nagoya 464-01

Japan

email: umemura@math.nagoya-u.ac.jp 